\title{
Viscoelastic Anisotropic Finite Element Mixture Model of Articular Cartilage USing Viscoelastic Collagen Fibers AND Validation with Stress Relaxation Data
}

\author{
A Thesis \\ presented to \\ the Faculty of California Polytechnic State University, \\ San Luis Obispo
}

In Partial Fulfillment

of the Requirements for the Degree

Master of Science in Mechanical Engineering

by

Matthew Alexander Griebel

June 2012 
(C) 2012

Matthew Alexander Griebel

ALL RIGHTS RESERVED 


\section{Committee Membership}

$\begin{array}{ll}\text { Title: } & \text { Viscoelastic Anisotropic Finite Element } \\ & \text { Mixture Model of Articular Cartilage } \\ & \text { Using Viscoelastic Collagen fibers and } \\ & \text { Validation with Stress Relaxation Data }\end{array}$

AUTHOR: $\quad$ Matthew Alexander Griebel

DATE SUBMITTED: June 2012

COMMITEE CHAIR: Stephen Klisch, Professor

COMMITTEE MEMBER: $\quad$ Scott Hazelwood, Associate Professor

COMMITTEE MEMBER: Tom Mase, Associate Professor 


\begin{abstract}
Viscoelastic Anisotropic Finite Element Mixture Model of

Articular Cartilage using Viscoelastic Collagen fibers and Validation with Stress Relaxation Data
\end{abstract}

Matthew Alexander Griebel

Experimental results show that collagen fibers exhibit stress relaxation under tension and a highly anisotropic distribution. To further develop the earlier model of Stender [1], the collagen constituent was updated to reflect its intrinsic viscoelasticity and anisotropic distribution, and integrated with an existing mixture model with glycosaminoglycans and ground substance matrix. A two-term Prony series expansion of the quasi-linear viscoelastic model was chosen to model the viscoelastic properties of the collagen fibers. Material parameters were determined by using the simplex method to minimize the sum of squared errors between model results and experimental stress relaxation data of tissue in tension. Collagen elastic fiber modulus was calculated by fitting to the equilibrium data and viscoelastic parameters were determined by fitting to the relaxation curve. Results of newborn $(\sim 1-3$ week old) untreated bovine articular cartilage explants from the patellar femoral groove as well as explants cultured in transforming growth factor- $\beta 1$ (TGF- $\beta 1$ ), from both the superficial $(\sim 0-0.5 \mathrm{~mm}$ from the articular surface) and middle $(\sim 0.5-1.0 \mathrm{~mm}$ from the articular surface) layers were compared to examine the effects of TGF$\beta 1$. TGF- $\beta 1$ has been shown to maintain or even enhance mechanical properties of articular cartilage in compression and tension $[2,3]$ and this study continues with the hope that it may be used to improve tissue engineering of mature cartilage to better survive implantation in vivo for the successful repair of articular cartilage defects. Results show that TGF- $\beta 1$ has a maturational effect on collagen, causing the tissue to become stiffer through an increase in elastic collagen fiber modulus and less viscous through shorter relaxation time and less stress relaxation (tissue retained a higher percentage of residual stress). The results of this study further advance the understanding of the effects of location and treatment with TGF- $\beta 1$.

Keywords: viscoelastic, cartilage, quasi-linear, simplex, standard linear solid 


\section{ACKNOWLEDGEMENTS}

First, I would like to acknowledge professors Steve Klisch and Scott Hazelwood. Their in-exhaustive support and knowledge were a priceless source of help throughout my project. I would also like to thank Kevin Yamauchi and Matteo Taffetani who would allow me to bounce ideas around, check my code, give me suggestions, and help me take a break from the seemingly endless work around me. Mike Stender also proved an enormous help, guiding me towards understanding numerous times. Mike and Kevin also graciously agreed to help edit and review my thesis.

Nathan Balcom's work in organizing and gathering experimental data was definitely an enormous help at the beginning of my project.

I'd also like to thank my family for supporting me while I finished this seemingly never ending project.

Lastly I'd like to thank my fianceé Pilar. Thanks for sticking with me and dealing with living so far apart. Thanks for the support and the help.

This study was funded in part by the NIH. 


\section{TABle of Contents}

LisT OF TABLES vii

List OF Figures

1 INTRODUCTION

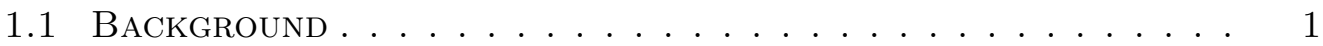

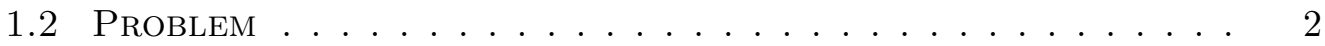

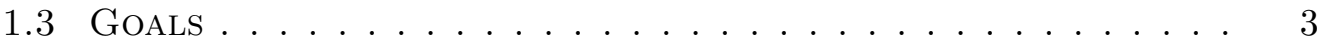

2 Literature Review 4

2.1 Articular Cartilage Composition .............. 4

2.2 Articular Cartilage Models . . . . . . . . . . . . 4

2.3 Collagen fiber Viscollasticity . . . . . . . . . . . 5

2.4 Anisotropic fiber Distribution . . . . . . . . . . . 6

2.5 Finite Element Analysis . . . . . . . . . . . . . . . . . 7

$\begin{array}{llr}3 & \text { THEORY } & \mathbf{8}\end{array}$

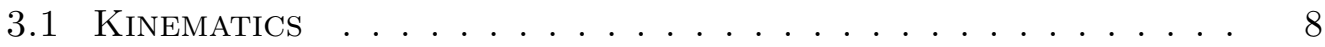

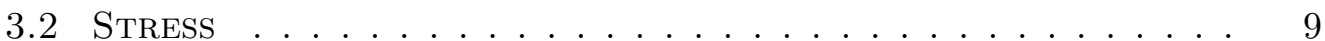

3.3 Model Constituents . . . . . . . . . . . . . . . . . 10

3.3.1 Proteoglycan Modeling . . . . . . . . . . . . . . . . . . 11

3.3.2 Collagen Modeling . . . . . . . . . . . . . . . . . 11

3.3.3 Ground Substance Matrix . . . . . . . . . . . . . . . . 13

3.4 Viscollasticity . . . . . . . . . . . . . . . . . . . 14

3.5 Model Constraints . . . . . . . . . . . . . . 16

3.5.1 Solid Matrix . . . . . . . . . . . . . . . 16

3.5 .2 Immobility . . . . . . . . . . . . . . . . . . . . . . 17

3.5.3 Stress Balance . . . . . . . . . . . . . . . . 18

4 Methods $r$

4.1 Experimental DATA . . . . . . . . . . . . . . . . . . 19

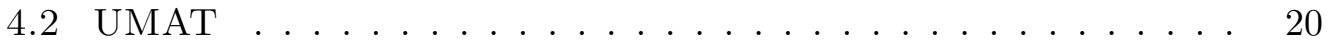

4.2.1 Quasi-Linear Viscoelastic Model . . . . . . . . . . . . . 21

4.3 Anisotropic Distribution . . . . . . . . . . . . . . . . . 22

4.4 SWELling AND CALCULATING STRAin . . . . . . . . . . . . 24

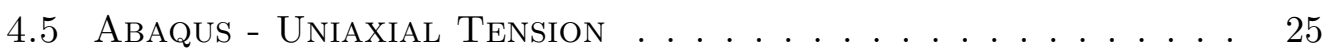

4.6 Simplex . . . . . . . . . . . . . . . . . . . . . . . . 27

4.6.1 Simplex Validation . . . . . . . . . . . . . . . 27

4.6 .2 Validation Results . . . . . . . . . . . . . . . . . 31

4.6.3 Simplex Application . . . . . . . . . . . . . . . . 32

4.7 DATA SETS . . . . . . . . . . . . . . . . . . 34

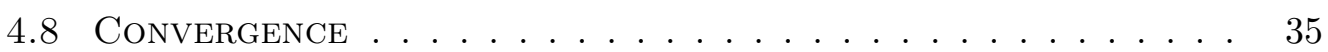

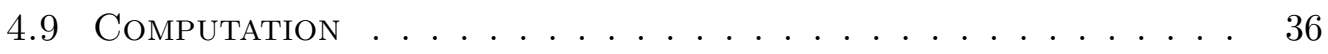

4.10 Statistical Analysis . . . . . . . . . . . . 36

$\begin{array}{llrl}5 & \text { Results } & \mathbf{3 7}\end{array}$ 
6 Discussion and Conclusions $\quad \mathbf{4 5}$

$\begin{array}{lr}\text { REFERENCES } & 49\end{array}$

A Derivations $\quad \mathbf{5 9}$

A.1 Two-Term Prony Series Expansion of the QLV . . . . . . 59

$\begin{array}{lr}\text { B Convergence Study } & \mathbf{6 4}\end{array}$

$\begin{array}{lrl}\text { C Permeability Study } & \mathbf{6 5}\end{array}$

$\begin{array}{lr}\text { D Tables and Figures } & \mathbf{6 6}\end{array}$

E Standard Linear Solid - Pilot Study $\quad \mathbf{7 4}$

E.1 BACKGROUnd . . . . . . . . . . . . . . . . . . . 74

E.2 Stress and Elasticity . . . . . . . . . . . . . . 74

E.3 Exact Solution to Differential Equation . . . . . . . . . . 79

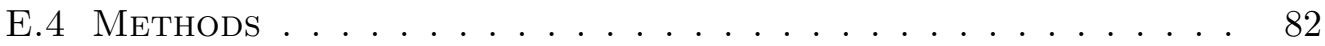

E.5 Results . . . . . . . . . . . . . . . . . . . . 83

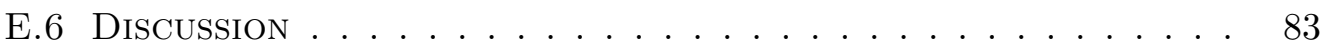




\section{LIST OF TABLES}

1 Average biochemical data. . . . . . . . . . . . . . . 20

2 Starting vertices of the average tissue samples for the simplex optimization method. . . . . . . . . . . . . . . . 34

$3 \quad$ Number of samples from each tissue group. . . . . . . . . . . . . . 35

4 Viscoelastic parameters for the QLV model for different average tissue groups. . . . . . . . . . . . . . . . . . 37

$5 \quad$ Overall viscoelastic response of each average tissue source and model. 37

6 Average elastic parameters for COL from each individual specimen. 40

7 Average viscoelastic parameters for the QLV model from each individual specimen. . . . . . . . . . . . . . . . . . 40

8 Specimen specific biochemical data. . . . . . . . . . . . . 66

9 Specimen specific simplex optimization results. . . . . . . . . . . . . 67

10 Specimen specific generalized relaxation results. . . . . . . . . . . 68

11 Viscoelastic parameters for the QLV model for different tissues groups and tare considerations. . . . . . . . . . . . . 73 


\section{LisT OF FigURES}

1 Arcade model of Benninghoff. . . . . . . . . . . . . . . . . 6

2 Material point showing spherical coordinate system and collagen fiber volume. . . . . . . . . . . . . . . . . . . . 12

3 Reference configuration of constituents and solid matrix. . . . . . . . 17

4 Experimental reference configuration and Abaqus. . . . . . . . . . 25

5 Graphical representation of experimental procedure. . . . . . . . . 26

6 The model used in an Abaqus simulation of uniaxial tension. . . . . 26

7 The movements of the simplex during minimization. . . . . . . . . . 27

8 Visualization of the simplex movements applied to the 2-variable Rosenbrock function. . . . . . . . . . . . . . . . . . . 31

9 Visualization of the same simplex movements (Figure 8) across the 3D surface of the Rosenbrock function. . . . . . . . . . . . . . . 31

10 Visualization of the simplex movements applied to the 3-variable Fletcher-Powell helical valley. . . . . . . . . . . . . . . . . 32

11 Decimation used in optimization. . . . . . . . . . . . . . 33

12 Fit of average untreated superficial layer specimens. . . . . . . . . 38

13 Fit of average untreated middle layer specimens. . . . . . . . . . . 38

14 Fit of average superficial layer specimens treated for 12 days in TGF- $\beta 1.39$

15 Fit of average middle layer specimens treated for 12 days in TGF- $\beta 1 . \quad 39$

16 Effect of TGF- $\beta 1$ on D0-S and D0-M elastic collagen fiber modulus. 41

17 Effect of TGF- $\beta 1$ on D0-S and D0-M viscoelastic parameter $g_{1}$ (the long term relaxation coefficient). . . . . . . . . . . . . . 41

18 Effect of TGF- $\beta 1$ on D0-S and D0-M viscoelastic parameter $\tau_{1}$ (the long term relaxation time constant). . . . . . . . . . . . . . 42

19 Effect of TGF- $\beta 1$ on D0-S and D0-M viscoelastic parameter $g_{2}$ (the short term relaxation coefficient). . . . . . . . . . . . . 42

20 Effect of TGF- $\beta 1$ on D0-S and D0-M viscoelastic parameter $\tau_{2}$ (the short term relaxation time constant). . . . . . . . . . . . . . 43

21 Individual tissue specimen fits. . . . . . . . . . . . . . . . . . . . . 44

22 Mesh convergence study. . . . . . . . . . . . . . . . 64

23 Comparison of D0-S QLV model when including permeability and when not including permeability in tension stress relaxation modeling. 65

24 Comparison of D0-M QLV model when including permeability and when not including permeability in tension stress relaxation modeling. 65

25 Comparison of QLV models curve fit to stress relaxation data from D0 superficial layer bovine articular cartilage under tensile load. . . 69

26 Comparison of QLV models curve fit to stress relaxation data from D0 middle layer bovine articular cartilage under tensile load. . . . . 70

27 Comparison of QLV models curve fit to stress relaxation data from D12-TGF- $\beta 1$ superficial layer bovine articular cartilage under tensile load. . . . . . . . . . . . . . . . . .

28 Comparison of QLV models curve fit to stress relaxation data from D12-TGF- $\beta 1$ middle layer bovine articular cartilage under tensile load. 72

29 A linear spring with elastic modulus E . . . . . . . . . . . . . . 74

30 A linear damper with coefficient $\eta \ldots \ldots \ldots$. . . . . . . . . 75

31 A standard linear solid (SLS) model. . . . . . . . . . . . . . . 75 
32 Standard linear solid model curve fit to average D0-M stress relaxation data. . . . . . . . . . . . . . . . . 83 


\section{INTRODUCTION}

\subsection{BACKGROUND}

Articular cartilage (AC) is a low-friction, load bearing, avascular biological tissue found between articulating surfaces of bones of humans and other animals $[4,5]$. It allows joints to articulate smoothly and transmits loads between bones in contact. $\mathrm{AC}$ injury, disease, and age can lead to irreparable damage, degradation, and pain. Arthritis, a degenerative condition marked by progressive loss of cartilage thickness and integrity, has become the leading medical cost in the United States; with arthritis affecting roughly 50 million people, annual costs have reached approximately 130 billion dollars [6].

Natural repair of AC is ineffective, most likely due to its avascularity and low metabolic activity of its cells (i.e chondrocytes). The lack of a natural repair response in $\mathrm{AC}$ has led physicians and scientists to seek out an effective treatment to restore functionality to the joint. Current treatments include physical therapy (for minor injuries and prevention), removal of problematic tissue (for more substantial injury), and total joint replacement (for major injuries) [7,8]. Removal of tissue and total joint replacement are invasive, unlikely to completely remedy the problem, and are

ultimately temporary [9]. Rising costs, along with patient comfort, have driven the need for a procedure that is cost effective, minimally invasive, and mechanically sound.

One of the solutions proposed is using tissue engineered AC. Tissue engineered $\mathrm{AC}$ could be grown in vitro and used in vivo to repair locally damaged AC or replace an entire joint surface. This presents a rather complex problem, however, as $\mathrm{AC}$ is by no means a simple engineering material. $\mathrm{AC}$ has been shown to exhibit a highly asymmetric tensile-compressive response $[10,11]$, depth dependent characteristics $[12,13]$, age dependent characteristics [14], as well as a highly viscoelastic response to various loading conditions $[15,16]$ and typically large deformations. For tissue engineered AC to result in a consistently successful repair strategy, the geometrical, biomechanical, biochemical, and tribological properties would need to be 
similar to the native tissue surrounding the implant to protect the chondrocytes from abnormal, damaging loads.

Recent work by the Cal Poly Cartilage Biomechanics Group (CPCBG) has striven to understand and model the complex nature of in vivo $\mathrm{AC}$ as well as the in vitro growth and remodeling of graft tissue aimed at repair of in vivo AC [17]. Current repair strategies include transplanted osteochondral auto- and allografts, and tissue engineered constructs $[8,18,19]$. The main difference between these strategies is the immediate load bearing properties of the implant and the post-operative rehabilitation time. Implants with geometrical, biomechanical, biochemical, and tribological properties identical to the in vivo properties are needed to facilitate normal joint biomechanics. This necessitates accurate replication of $\mathrm{AC}$ explant properties to aid in a consistently successful repair strategy.

\subsection{Problem}

The CPCBG subjected explants of untreated AC, and explants treated for twelve days in transforming growth factor- $\beta 1$ (TGF- $\beta 1$ ) according to procedures set forth by [20], to various mechanical tests including confined compression (CC), unconfined compression (UCC), and uniaxial tension (UT) [2]. After ramp loading, stress in the specimens relaxed to a non-zero value. This response confirms the viscoelastic nature of AC as previously reported [16, 21, 22]. A recent constitutive model was developed by Stender [1] to model AC and provide a basis for later work by Kam [23] to model growth and remodeling of AC. Although Kam's work tackles some of the viscoelastic nature of AC by including permeability [24], neither his nor Stender's work attempted to model the intrinsic viscoelasticity of the tissue in tension. Previous work by Thomas [69] looked at the poroviscoelasticity of AC, but modeled the tissue as a constituent model of only collagen (COL) fiber and glycosaminoglycan (GAG) components (based on the model developed by Klisch [25]) and was used to study GAG depletion and the role of COL-GAG interactions. The new models developed by Stender and Kam model AC as a mixture of a continuous distribution 
of isotropic COL fibers, a GAG component, as well as a ground substance matrix (MAT) component (accounting for all other material constituents not captured by the COL network or GAG).

\subsection{GOALS}

The objective of this work is to develop a viscoelastic model for COL fibers in the previously developed model [1] which consists of a continuous distribution of COL fibers, along with a GAG and MAT component, and use the model to characterize the effects of location (patellarfemoral groove, superficial $(\sim 0-0.5 \mathrm{~mm})$ and middle $(\sim 0.5-1.0 \mathrm{~mm})$ layers $)$ and treatment in TGF- $\beta 1$ of newborn $(\sim 1-3$ weeks $)$ bovine AC. The hypothesis is that both location (superficial vs. middle) and treatment (untreated vs. treated) affect COL viscoelasticity (by changing both the elastic COL fiber modulus and the viscous material parameters set forth in the viscoelastic model used). This hypothesis is tested by curve fitting the data to tension stress relaxation experiments and using statistical analysis on the predicted model parameters. 


\section{Literature RevieW}

\subsection{Articular Cartilage Composition}

Articular cartilage is a triphasic material composed of a porous matrix, water, and ions. The porous matrix consists of type II collagen ( $\sim 10-20 \%$ wet weight), proteoglycans ( $\sim 4-7 \%$ wet weight), and extracellular matrix (describing other proteins). Collagen is primarily responsible for resisting tension and shear, and is grouped into bundles called fibers. Proteoglycans are primarily responsible for resisting compression and are complex macromolecules consisting of a long protein chain with polysaccharide links. Many of these proteoglycans have glycosaminoglycan links which in solution form negatively charged ions. This negative charge causes proteoglycans to swell, which is resisted by the collagen network and creates an internal swelling stress. The negative charge also draws water in by osmosis. Water accounts for $\sim 70-90 \%$ of the wet weight, and since cartilage has a low permeability (i.e. a high resistance to fluid flow through the molecular matrix), the tissue exhibits considerable flow-dependent viscoelasticity (typically only in compression) [26].

\subsection{Articular Cartilage Models}

The linear biphasic mixture theory derived by Mow et al. [27] has been used to model creep and stress-relaxation of articular cartilage [14, 27, 28]. The derivation is based on modern mixture theory developed by Truesdell and Toupin [29], Green and Naghdi [30, 31], Bowen [32], and Müller [33], and used the theory of Craine et al. [34] along with the constraint of intrinsic incompressibility [35]. Large deformations were predicted in the solid matrix (SM) and so a finite deformation theory was used to derive nonlinear constitutive restrictions [28]. Earlier finite deformation models were developed by Holmes and Mow [36] and Ateshian et al. [37], while work by Klisch and Lotz [38] was done to develop a "special" mixture theory for cartilage that incorporates an intrinsically incompressible mixture of an elastic solid and an inviscid fluid. 
The model by Stender [1] extends previous finite deformations models to include a continuous distribution of COL fibers and a separate component to account for shear properties of AC. The SM is composed of three constituents: GAG, COL, and MAT. Stender's work developed a polyconvex continuum-level proteoglycan Cauchy stress function based on the continuum electromechanical Poisson-Boltzman cell model. This was combined with a nonlinear elastic collagen fiber distribution model and an isotropic hyperelastic compressible NeoHookean material to model the MAT (which is intended to account for the mechanical response of other SM components not accounted for in GAG or COL). For a more in depth look at GAG and MAT material modeling please see [1].

\subsection{Collagen fiber Viscoelasticity}

The viscoelasticity of $\mathrm{AC}$ has been modeled as both dependent and independent of fluid flow $[21,24,39]$. While poroelastic models are necessary to accurately model relaxation in compression tests [40] they are less important in modeling tension tests where pressure gradients are negligible and the intrinsic viscoelasticity of COL governs the relaxation characteristics $[22,41]$. These results necessitate a model that is capable of modeling stress relaxation as both dependent and independent of fluid flow. Such biphasic models were first proposed by Mak [21], creating a biphasic poroviscoelastic model using the biphasic theory of Mow et al. [27] and the integral-type linear viscoelastic model proposed by Fung [42]. Biphasic models have been implemented in several different studies $[43,44,45]$. However, Li et al. [41] suggests that the effects of fluid flow are negligible in uniaxial tension and that only the intrinsic viscoelasticity of the collagen fibers affects the tissue's relaxation properties in extension.

The intrinsic viscoelasticity of collagen fibers has been modeled with linear [46, 47], quasi-linear [21, 22, 42], and nonlinear viscoelastic formulas [45, 48, 49]. The quasi-linear viscoelastic (QLV) model was first proposed by Fung [42] and has been used extensively [21, 69, 50, 51, 52]. Vena et al. [51] and Thomas [69] implemented 
a constituent based viscoelastic model, where GAGs and COLs may have different QLV properties, and a time discretization procedure on the QLV to facilitate finite element modeling similar to work by Suh and Bai [43] and Puso and Weiss [53].

\subsection{Anisotropic Fiber Distribution}

Experimental results show that cartilage is highly anisotropic. Along the depth of mature (i.e. adult) cartilage, fibers are oriented parallel to the surface in the superficial zone, randomly in the middle zone, and perpendicular to the surface in the deep zone [54]. Often this is represented with the arcade model of Benninghoff [55] (Figure 1). Recent studies have developed continuous fiber distribution functions to better describe the anisotropy of $\mathrm{AC}[56,57]$. Lei [56] examines a horizontal fiber alignment that uses a Gaussian function definition. A novel approach developed by Shirazi et al. uses true moduli of collagen fibers multiplied by differing volume fractions to represent a continuous distribution of fibers that can be applied to either isotropic or anisotropic distributions [58].

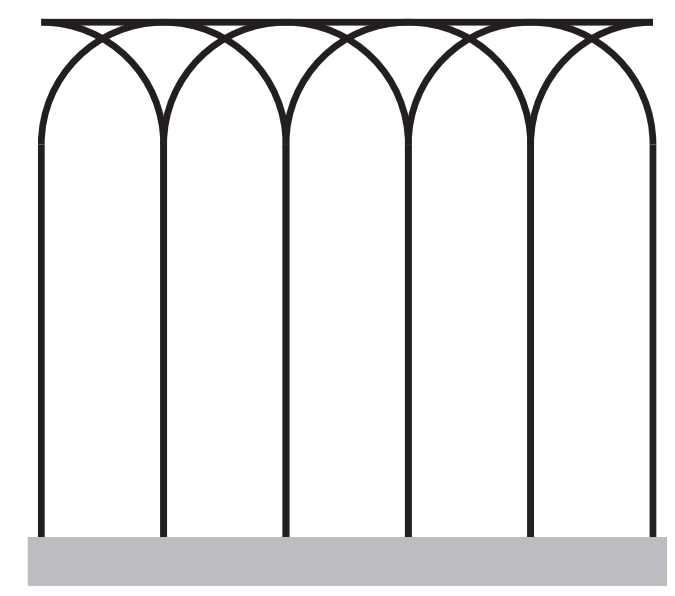

Figure 1: Arcade model of Benninghoff. This model shows collagen fibers that are parallel to the surface in the superficial layer, somewhat isotropic in the middle zone, and perpendicular in the deep zone.

Immature cartilage, on the other hand, exhibits a less heterogeneous distribution with fibers running mostly parallel to the articular surface through the depth [59, 60, 61]. Because of this difference in COL fiber distribution, care must be taken 
when generalizing a model across different AC tissue groups (species, location, and age).

\subsection{Finite Element Analysis}

Finite element analysis (FEA) has been successful in modeling the complicated behavior of articular cartilage [62]. Because of complex geometries, material properties, environmental interactions, and loading conditions, exact solutions are not possible for articular cartilage models. FEA allows for an approximation to the solution that can be used to gain insight and knowledge on the nature of AC. Abaqus [63] was chosen as the commercial finite element software to complete the analysis since it has been proven to successfully model biphasic tissues [64] and can model custom materials using the user defined material subroutine (UMAT) option. 


\section{THEORY}

A brief introduction to kinematics and continuum mechanics is provided for reference. A basic understanding of tensor calculus, along with indicial notation, is necessary for the following section. See $[65,66]$ for a more in depth look at the subject. Following that, the theory behind each model will be developed. Note that both direct (bold font) and indicial (subscripts representing values of 1, 2, or 3) will be used.

\subsection{Kinematics}

Consider a body $\beta$, with a reference configuration $\kappa_{o}$ at time $t_{o}$, that undergoes some displacement or deformation to occupy the current configuration $\kappa$ at time t. A point on $\beta$ has position vector $\boldsymbol{X}$ in the reference configuration and $\boldsymbol{x}$ in the current configuration. The invertible motion is described by the equation

$$
\boldsymbol{x}=\chi(\boldsymbol{X}, t) .
$$

The deformation gradient tensor can be defined using standard indicial notation as

$$
F_{i A}=x_{i, A}
$$

where comma notation is used to indicate a partial derivative (in Equation 2 with respect to position, i.e. $x_{i, A}$ represents partial derivative with respect to $X_{A}$ ). The Jacobian determinant of the deformation gradient tensor is defined as

$$
J=\operatorname{det}(\boldsymbol{F})=\left|\frac{\partial x_{i}}{\partial X_{A}}\right|
$$

where $\operatorname{det}(\cdot)$ is the determinant operator. Equation 3 guarantees the invertibility of the motion as long as

$$
J \neq 0
$$


The Right Cauchy-Green deformation tensor is defined as

$$
\boldsymbol{C}=\boldsymbol{F}^{T} \boldsymbol{F}
$$

from which we may define the Lagrangian finite strain tensor as

$$
\boldsymbol{E}=\frac{1}{2}(\boldsymbol{C}-\boldsymbol{I})
$$

where $\boldsymbol{I}$ is the identity tensor.

\section{$3.2 \quad$ STRESS}

The most well known equation for stress is Cauchy $(\boldsymbol{\sigma})$ stress which is a measure of force per unit area in the current configuration.

$$
t=\sigma^{T} \cdot n
$$

where $\boldsymbol{t}$ is the traction vector and $\boldsymbol{n}$ is the unit vector normal to the surface on which the traction acts.

The first Piola-Kirchhoff stress $(\boldsymbol{P})$ is defined as the force acting on the current configuration per unit area in the reference configuration (which is the form of stress often reported in experimental data as the reference configuration area is known while the current configuration area is not)

$$
\boldsymbol{t}_{o}=\boldsymbol{P} \cdot \boldsymbol{n}_{o}
$$

Cauchy stress is useful in analyzing small deformations but its rate (i.e $\frac{\mathrm{d} \sigma}{\mathrm{d} t}$ where $t$ is time) is not objective, thus it is often easier to use second Piola-Kirchoff stress whose rate is objective.The second Piola-Kirchhoff stress $(\boldsymbol{S})$ is defined as

$$
\boldsymbol{S}^{T} \cdot \boldsymbol{n}_{o}=\boldsymbol{F}^{-1} \cdot \boldsymbol{t}_{o}
$$


where $^{-1}$ is the inverse operator. The relationship between all three stress measures is

$$
J \boldsymbol{\sigma}=\boldsymbol{F} \boldsymbol{S} \boldsymbol{F}^{T}=\boldsymbol{P} \boldsymbol{F}^{T}
$$

Abaqus defines all stress in terms of Cauchy stress so care must be taken to convert to Cauchy stress before reporting to Abaqus.

Derivation of the elasticity tensor is also necessary for implementation in Abaqus. The elasticity tensor can be defined as

$$
\tilde{\mathbb{C}}=\frac{\partial \boldsymbol{S}}{\partial \boldsymbol{E}}=2 \frac{\partial \boldsymbol{S}}{\partial \boldsymbol{C}}
$$

This value is only acceptable for models with small deformations. For large deformations, the material Jacobian matrix for total form constitutive equations gives a generalized form that includes correctional rotation terms to increase convergence in problems with co-rotations. The results of the derivation lead to the definition of the Jacobian stiffness matrix in indicial notation as

$$
\begin{aligned}
\tilde{\mathbb{C}}_{i j k r}^{J a c}=\frac{1}{J}\left\{\left[\frac{J}{2}\left(\delta_{i k} \delta_{p r}+\delta_{p k} \delta_{i r}\right) T_{p j}\right.\right. & \left.+\frac{J}{2}\left(\delta_{j k} \delta_{n r}+\delta_{n k} \delta_{j r}\right) T_{i n}\right] \\
& \left.+\left[\tilde{\mathbb{C}}_{A B C D} F_{i A} F_{j B} F_{k C} F_{r D}\right]\right\}
\end{aligned}
$$

the details of which were developed by Pasquale Vena and Reza Shirazi and can be found in [1]. Note that $\widetilde{\mathbb{C}}_{A B C D}$ is the elasticity tensor described in Equation 11. Equation 12 is the form of the elasticity tensor reported to Abaqus in the UMAT.

\subsection{Model Constituents}

As previously mentioned, the model is composed of three separate constituents, proteoglycan (GAG), collagen (COL), and ground substance matrix (MAT). Each of the general theories for these constituents is presented here. 


\subsubsection{Proteoglycan Modeling}

Stender [1] developed a proteoglycan Cauchy stress function based on the PoissonBoltzman (PB) cell model for osmotic swelling pressure. After curve fitting the predictions of that model, the Cauchy stress can be shown to be

$$
\boldsymbol{T}^{G A G}=-\alpha_{1}\left(\rho^{G A G}\right)^{\alpha_{2}} \boldsymbol{I}
$$

where $\rho^{G A G}$ is the apparent GAG density in the current configuration, $\alpha_{1}$ and $\alpha_{2}$ material constants, and $\boldsymbol{I}$ is the identity tensor. The continuity equation

$$
\rho_{0}=J \rho,
$$

allows us to rewrite Equation 13 as

$$
\boldsymbol{T}^{G A G}=-\alpha_{1}\left(\frac{\rho_{0}^{G A G}}{J}\right)^{\alpha_{2}} \boldsymbol{I}
$$

Here, $\rho_{0}^{G A G}$ is the experimentally measured reference configuration GAG apparent density. Stender showed that $\alpha_{1}=2.87 \frac{\mathrm{N} \cdot \mathrm{ml} \mathrm{l}^{2.5}}{\mathrm{~m}^{2} \cdot \mathrm{mg}^{2.5}}$ and $\alpha_{2}=2.5$ resulted in a good fit $\left(R^{2}=0.98\right)$ to PB model swelling stress predictions and experimental data from Buschmann and Grodzinsky [67].

\subsubsection{Collagen Modeling}

COL is modeled as a continuous distribution of COL fibers using a volume fraction at all material points. The method was developed by Shirazi et al. [58] and described by Stender [1]. The method will be summarized here.

Consider a unit sphere (Figure 2) that is split up into differential pyramid elements. The total COL volume fraction is defined as

$$
\phi^{f}=\frac{V^{f}}{V}
$$




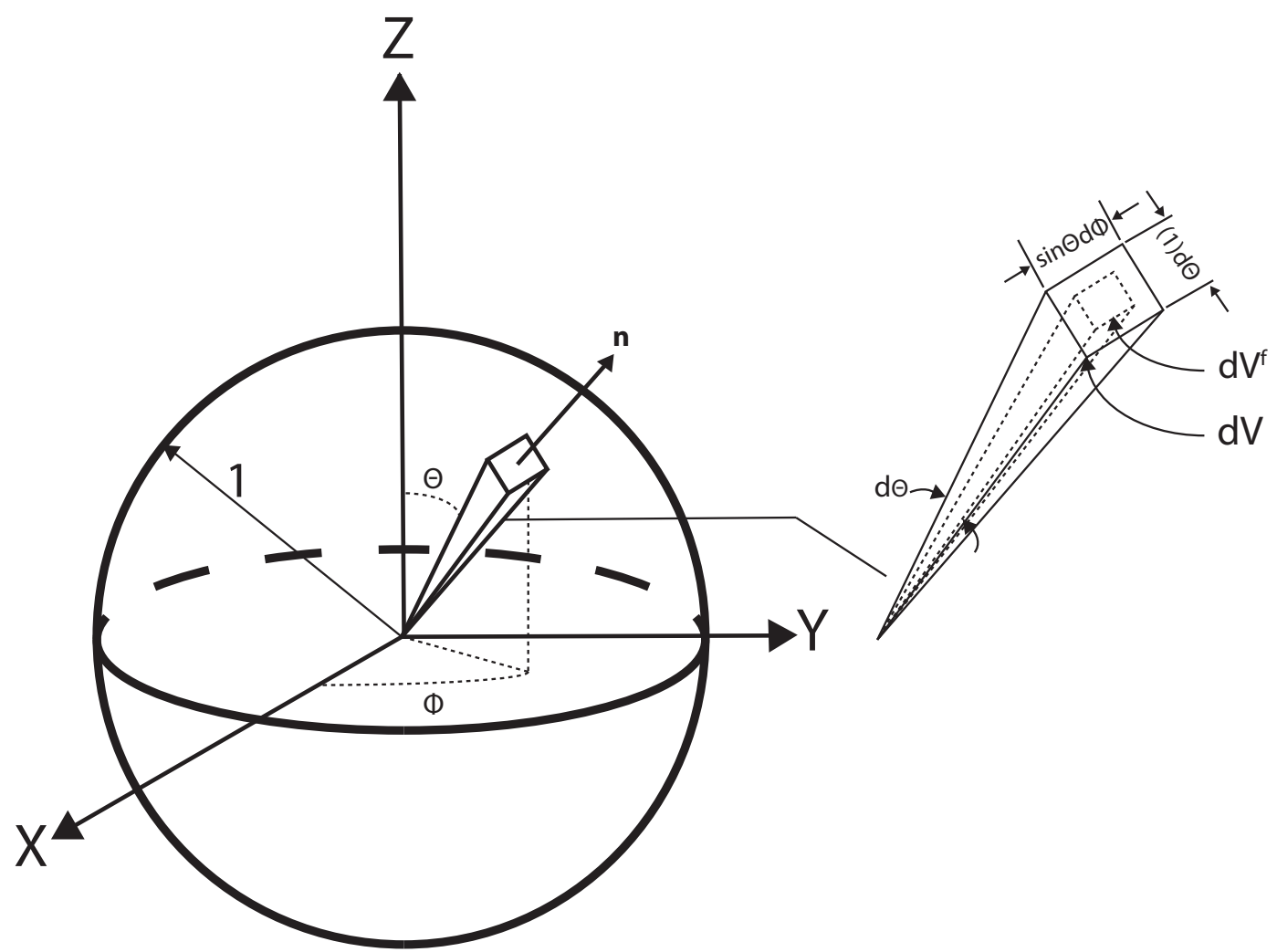

Figure 2: Material point showing spherical coordinate system and collagen fiber volume.

where $V^{f}$ is the volume of COL in the unit sphere and $V$ is the volume of the unit sphere. A fiber volume fraction distribution function is then defined to account for a continuous distribution of fibers

$$
R(\theta, \phi)=\frac{\phi_{n}^{f}}{V}
$$

where $\phi_{n}^{f}$ is the directional fiber volume fraction in direction $\boldsymbol{n}$

$$
\phi_{n}^{f}=\frac{\mathrm{d} V^{f}}{\mathrm{~d} V}
$$

Here, $\mathrm{d} V^{f}$ is the fiber volume in $\mathrm{d} V$ and $\mathrm{d} V=\frac{1}{3} \sin \theta \mathrm{d} \theta \mathrm{d} \phi$ (Figure 2). Equation 17 is then integrated over the volume of the unit sphere

$$
\int_{V} R(\theta, \phi) \mathrm{d} V
$$


to get the total fiber volume. Using quantitative polarized light microscopy (qPLM) data and methods developed by Yamauchi [68], a discrete distribution function was developed for implementation into FEA.

Shirazi et al. [58] then used Equation 19 to develop the COL stress and (along with Equation 11) the COL elasticity tensor.

$$
\begin{aligned}
\boldsymbol{\sigma}^{C O L} & =\int_{V} R(\theta, \phi) H \sigma_{N} \boldsymbol{N} \otimes \boldsymbol{N} \mathrm{d} V \\
\tilde{\mathbb{C}}^{C O L} & =\int_{V} R(\theta, \phi) H \frac{\partial \sigma_{N}}{\partial \boldsymbol{E}} \boldsymbol{N} \otimes \boldsymbol{N} \mathrm{d} V
\end{aligned}
$$

where $\sigma_{N}$ is the $1 \mathrm{D}$ fiber stress, $N$ is the unit normal in the reference configuration, $\otimes$ is the dyadic product, and $H$ is the heavyside step function to insure COL only carries tension (Equation 22)

$$
H= \begin{cases}0 & E_{N}<0 \\ 1 & E_{N} \geq 0\end{cases}
$$

where $E_{N}$ is the Lagrangian strain in the fiber direction

$$
E_{N}=\boldsymbol{E} \boldsymbol{N} \cdot \boldsymbol{N}
$$

\subsubsection{Ground Substance Matrix}

MAT is meant to model all of the SM properties not contained within the GAG or COL constitutive relationships. The model chosen to govern MAT is the NeoHookean solid. Stender [1] shows that the second Piola-Kirchhoff stress for this material is

$$
\boldsymbol{S}^{M A T}=\mu\left(\boldsymbol{I}-\boldsymbol{C}^{-1}\right)
$$


where $\mu$ is the MAT shear modulus. This is transformed in Cauchy stress and becomes

$$
\boldsymbol{T}^{M A T}=\frac{1}{J} \boldsymbol{F}\left[\mu\left(\boldsymbol{I}-\boldsymbol{C}^{-1}\right)\right] \boldsymbol{F} .
$$

\subsection{Viscoelasticity}

Fung [42] introduced the quasi-linear viscoelastic (QLV) model to calculate stress by way of a linear convolution integral

$$
\sigma(t)=\int_{-\infty}^{t} g(t-\xi) \frac{\mathrm{d} \sigma^{e}}{\mathrm{~d} \xi} \mathrm{d} \xi
$$

where $\sigma$ is uniaxial stress, $g(t)$ is the relaxation function, and $\sigma^{e}$ is the elastic stress. This model is considered quasi-linear because the dependence of response on loading history can be obtained from a linear convolution integral.

Assuming that all stress before $t=0$ is zero, that the initial stress is not zero (i.e. $\boldsymbol{\sigma}(0) \neq 0$ ), and that all viscoelastic stress is fiber stress (so the derivation can be done in scalar form) we will rewrite Equation 26 in terms of elastic stress as

$$
\sigma^{v}(t)=\sigma^{e}(0)+\int_{0}^{t} g(t-\xi) \frac{\mathrm{d} \sigma^{e}}{\mathrm{~d} \xi} \mathrm{d} \xi
$$

where $\sigma^{v}$ is the viscoelastic fiber stress.

The relaxation function is represented as a two-term Prony series expansion

$$
g(t)=g_{\infty}+g_{1} e^{-t / \tau_{1}}+g_{2} e^{-t / \tau_{2}}
$$

To implement Equation 27 into a finite element code, the integral equation needs to be changed into a time incrementation algorithm which provides the stress at the current time based on the stress at the previous time. The time discretization scheme that follows was proposed by Vena [51] and is similar to Puso and Weiss [53]. See Thomas et al. for a similar derivation to the one presented here [69]. 
The fiber stress at the current time is calculated as

$$
\sigma^{v}(t+\Delta t)=\sigma^{e}(0)+\int_{0}^{t+\Delta t} g(t+\Delta t-\xi) \frac{\mathrm{d} \sigma^{e}}{\mathrm{~d} \xi} \mathrm{d} \xi
$$

where the relaxation function is now

$$
g(t+\Delta t-\xi)=g_{\infty}+g_{1} e^{[\xi-(t+\Delta t)] / \tau_{1}}+g_{2} e^{[\xi-(t+\Delta t)] / \tau_{2}} .
$$

Following the derivation of Appendix A.1 we can calculate the fiber stress at the current time as

$$
\begin{aligned}
\sigma^{v}(t+\Delta t)=\sigma_{0}^{e} & +g_{\infty}\left(\sigma^{e}(t)-\sigma^{e}(0)\right)+e^{\frac{-\Delta t}{\tau_{1}}} c_{1}(t)+e^{\frac{-\Delta t}{\tau_{2}}} c_{2}(t) \\
+ & \frac{\sigma^{e}(t+\Delta t)-\sigma^{e}(t)}{\Delta t} \begin{array}{r}
{\left[g_{\infty} \Delta t\right.} \\
+g_{1} \tau_{1}\left(1-e^{-\Delta t / \tau_{1}}\right) \\
\left.+g_{2} \tau_{2}\left(1-e^{-\Delta t / \tau_{2}}\right)\right]
\end{array}
\end{aligned}
$$

where $c_{1}(t)$ and $c_{2}(t)$ are defined as

$$
\begin{gathered}
c_{1}(t)=e^{-\Delta t / \tau_{1}} c_{1}(t-\Delta t)+\frac{\sigma^{e}(t)-\sigma^{e}(t-\Delta t)}{\Delta t}\left[g_{1} \tau_{1}\left(1-e^{-\Delta t / \tau_{1}}\right)\right] \\
c_{2}(t)=e^{-\Delta t / \tau_{2}} c_{2}(t-\Delta t)+\frac{\sigma^{e}(t)-\sigma^{e}(t-\Delta t)}{\Delta t}\left[g_{2} \tau_{2}\left(1-e^{-\Delta t / \tau_{2}}\right)\right] .
\end{gathered}
$$

This result is input into the spherical integration to solve for COL stress at the current time

$$
\boldsymbol{\sigma}^{C O L}(t+\Delta t)=\int_{V} R H \sigma^{v}(t+\Delta t)(\boldsymbol{N} \otimes \boldsymbol{N}) \mathrm{d} V
$$

The COL elasticity tensor at the current time can be described as

$$
\tilde{\mathbb{C}}^{C O L}(t+\Delta t)=\int_{V} R H \frac{\partial \sigma^{v}(t+\Delta t)}{\partial \boldsymbol{E}(t+\Delta t)}(\boldsymbol{N} \otimes \boldsymbol{N}) \mathrm{d} V
$$


where $\frac{\partial \sigma^{v}}{\partial \boldsymbol{E}}$ can be redefined as

$$
\frac{\partial \sigma^{v}}{\partial \boldsymbol{E}}=\frac{\partial \sigma^{v}}{\partial E_{N}} \frac{\partial E_{N}}{\partial \boldsymbol{E}}
$$

The partial derivative of $E_{N}$ with respect to $\boldsymbol{E}$ is

$$
\frac{\partial E_{N}}{\partial \boldsymbol{E}}=\boldsymbol{N} \otimes \boldsymbol{N}
$$

If we take the partial derivative of Equation 31 with respect to fiber Lagrangian strain

$$
\frac{\partial \sigma^{v}(t+\Delta t)}{\partial E_{N}(t+\Delta t)}=\left[g_{\infty}+\frac{g_{1} \tau_{1}}{\Delta t}\left(1-e^{-\Delta t / \tau_{1}}\right)+\frac{g_{2} \tau_{2}}{\Delta t}\left(1-e^{\Delta t / \tau_{2}}\right)\right] \frac{\partial \sigma^{e}(t+\Delta t)}{\partial E_{N}(t+\Delta t)}
$$

Let us define material constant $c$ as

$$
c=g_{\infty}+\frac{g_{1} \tau_{1}}{\Delta t}\left(1-e^{-\Delta t / \tau_{1}}\right)+\frac{g_{2} \tau_{2}}{\Delta t}\left(1-e^{-\Delta t / \tau_{2}}\right)
$$

so that the collagen elasticity tensor $\tilde{\mathbb{C}}$ becomes

$$
\tilde{\mathbb{C}}^{C O L}=\int_{V} R H c \frac{\partial \sigma^{e}}{\partial E_{N}}(\boldsymbol{N} \otimes \boldsymbol{N}) \otimes(\boldsymbol{N} \otimes \boldsymbol{N}) .
$$

\subsection{Model Constraints}

Sections 3.5.1-3.5.3 are covered in [1]. They are summarized here for completeness and continuity.

\subsubsection{SOLID Matrix}

The solid matrix (SM) of AC is composed of GAG, COL, and MAT constituents.

Each constituent has initial stress-free reference configurations $\kappa_{0}^{G A G}, \kappa_{0}^{C O L}$, and $\kappa_{0}^{M A T}$ respectively (Figure 3 ). 


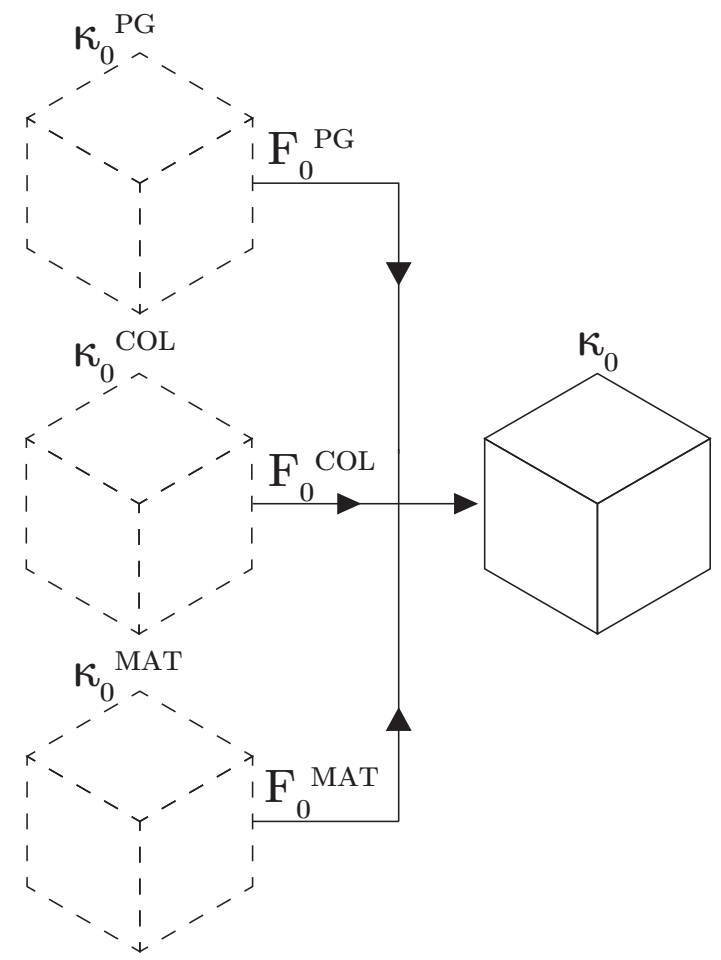

Figure 3: Reference configuration of constituents and solid matrix. Each constituent has an initial reference configuration that undergoes an initial deformation in order to satisfy the initial stress free reference configuration for the solid matrix.

The GAG constituent exhibits an intrinsic spherical swelling stress in the constituent reference configuration causing the other constituents to initially deform to meet the required stress free SM reference configuration $\kappa_{0}$. This swelling deformation leads to a tensile stress in the COL and MAT constituents $[69,70]$.

\subsubsection{ImMoBILITY}

The immobility constrain assumes that GAG, COL, and MAT molecules are bound to the SM and therefore each constituent's deformation gradient is equal to the SM deformation gradient relative to the SM reference configuration $\left(\kappa_{0}\right)$

$$
\mathbf{F}^{G A G}=\mathbf{F}^{C O L}=\mathbf{F}^{M A T}=\mathbf{F}^{S M} .
$$

Relative to their respective reference configurations, each constituent can have a deformation gradient that differs from other constituents. The immobility constraint 
allows for the determination of the each constituent deformation relative to $\kappa_{0}$. The immobility constraint has not been conclusively verified, but it has been successfully implemented in several studies $[70,71,72]$.

\subsubsection{Stress BalanCE}

Stress balance is a common assumption in continuum mixture theory [73]. The hypothesis states that the SM stress is equal to the sum of each of the constituent stresses

$$
\mathbf{T}^{S M}=\mathbf{T}^{G A G}+\mathbf{T}^{C O L}+\mathbf{T}^{M A T} .
$$

This hypothesis allows for non-zero constituent stress in the stress free SM reference configuration $\left(\kappa_{0}\right)$ so that the SM stress remains zero in that configuration. 


\section{Methods}

\subsection{EXPERIMENTAL DATA}

Experimental stress relaxation and biochemical data came from Stender et al. [3]. Full thickness bovine newborn ( $\sim 1-3$ weeks) calf articular cartilage blocks were harvested from the patellofemoral groove. Superficial ( $\sim 0-0.5 \mathrm{~mm}$ from the surface) and middle ( $\sim 0.5-1.0 \mathrm{~mm}$ from the surface) layers were sliced from the articular surface using a vibratome. Samples were cultured in TGF- $\beta 1$ and Dulbecco's modified Eagle's medium for twelve days in an incubator according to methods established by Asanbaeva et al. [20]. The slices were then punched into a dog-bone shape with a gauge length of $4 \mathrm{~mm}$ and width of $0.8 \mathrm{~mm}$. The long axis of the sample was oriented in the anterior posterior direction. Samples were stretched under displacement control to $5 \%$ tensile strain. Loading was performed over a $40 \mathrm{~s}$ period and then the sample was held in place and allowed to relax for $5000 \mathrm{~s}$. A two-term exponential decay was fit to the experimental data to determine equilibrium stress which was subsequently used to calculate the equilibrium secant modulus (total equilibrium stress divided by $5 \%$ strain). The specimens remained hydrated during the stress relaxation protocol by means of a phosphate buffered saline with protease inhibitors solution. qPLM data came from Stender et al. [74]. Average biochemical data can be found in Table 1. Specimen specific biochemical data can be found in Appendix D. 
Table 1: Average biochemical data for D0 (untreated) and D12-TGF- $\beta 1$ (treated) superficial and middle layers. Specimen specific data can be found in Appendix D. GAG and COL constituents are normalized to wet weight final. $\phi_{f}$ (COL volume fraction) is calculated from $\% W W_{f}$ of COL using the true density of COL $\left(1.436 \mathrm{~g} / \mathrm{cm}^{3}[75]\right)$. Values from $[3]$

\begin{tabular}{|c|c|c|c|}
\hline Tissue & $\begin{array}{c}\text { GAG } \\
\left(\% W W_{f}\right)\end{array}$ & $\begin{array}{c}\text { COL } \\
\left(\% W W_{f}\right)\end{array}$ & $\begin{array}{c}\phi_{f}^{\text {tot }} \\
(\% \text { total tissue volume })\end{array}$ \\
\hline D0-S & 3.113 & 7.053 & 4.91 \\
\hline D0-M & 5.054 & 8.837 & 6.15 \\
\hline D12-S & 5.197 & 5.209 & 3.63 \\
\hline D12-M & 4.518 & 4.417 & 3.08 \\
\hline
\end{tabular}

\subsection{UMAT}

Abaqus' user subroutine to define a material's mechanical behavior (UMAT) was used to define a custom mechanical constitutive behavior. The UMAT is called at all integration points and defines the stress and material Jacobian of the material at those integration points. Stress is defined as Cauchy stress within the UMAT, so calculations in other forms of stress must be converted before exiting the UMAT. An exact definition of the material Jacobian is not required for solution convergence, but the more accurate the definition, the faster a solution will be found. Since the strain variable used by the UMAT (STRAN) is log-strain, the Lagrangian strain must be calculated from the deformation gradient each time the UMAT is called. The deformation gradient is passed to the UMAT as DFGRD0 and DFGRD1 (the reference and current configuration deformation gradient respectively). The UMAT can also use solution-dependent state variables (STATEV) which must also be updated every time the UMAT is called. STATEVs can be used to define custom variables needed for calculation.

Stender [1] and Kam [23] developed a UMAT that was a nonlinear isotropic elastic mixture model. It contained the GAG and MAT descriptions of stress above (Section 3.3.1 and 3.3.3), along with an elastic constitutive model for COL. This UMAT was updated to reflect the viscoelastic model being applied to the collagen 
fibers. A new constitutive relationship for COL was coded based on the derivations presented above. In addition, anisotropy was added to the distribution of $\mathrm{COL}$ fibers.

For the Prony series expansion, it is necessary to save $c_{1}(t-\Delta t)$ and $c_{2}(t-\Delta t)$ for each fiber direction, $E(t-\Delta t)$ the Lagrangian strain tensor from the previous time increment, and $\Delta t$ the previous time increment. These are all saved and updated as STATEVs within the UMAT. For this model, $\mu$ - the solid matrix shear modulus was set to equal 0.001 to have continuity with Stender's [1] work and be able to use both conclusions from his and this study in future and continuing work.

\subsubsection{Quasi-Linear Viscoelastic Model}

The following replaced the elastic collagen loop in the UMAT to reflect the derivation of Section 3.4. First, the viscoelastic stress in the fiber direction (Equation 31) is rewritten as

$$
\begin{aligned}
\sigma^{v}(t+\Delta t) & =\sigma_{0}^{e}\left(1-g_{\infty}\right) \\
& +\sigma^{e}(t)\left[g_{\infty}-g_{\infty}-\frac{g_{1} \tau_{1}}{\Delta t}\left(1-e^{-\Delta t / \tau_{1}}\right)-\frac{g_{2} \tau_{2}}{\Delta t}\left(1-e^{-\Delta t / \tau_{2}}\right)\right] \\
& +\sigma^{e}(t+\Delta t)\left[g_{\infty}+\frac{g_{1} \tau_{1}}{\Delta t}\left(1-e^{-\Delta t / \tau_{1}}\right)-\frac{g_{2} \tau_{2}}{\Delta t}\left(1-e^{-\Delta t / \tau_{2}}\right)\right] \\
& +c_{1}(t) e^{-\Delta t / \tau_{1}}+c_{2}(t) e^{-\Delta t / \tau_{2}}
\end{aligned}
$$

where the superscripts $v$ and $e$ correspond to viscoelastic and elastic stress respectively. $c_{1}(t)$ and $c_{2}(t)$ are defined in equations 32 and 33 respectively. If we enforce the constraint that $g_{\infty}=1$ (so that the equilibrium stress converges to the elastic solution), the viscoelastic stress in the fiber direction becomes

$$
\begin{aligned}
\sigma^{v}(t+\Delta t)= & -\sigma^{e}(t)\left[\frac{g_{1} \tau_{1}}{\Delta t}\left(1-e^{-\Delta t / \tau_{1}}\right)+\frac{g_{2} \tau_{2}}{\Delta t}\left(1-e^{-\Delta t / \tau_{2}}\right)\right] \\
& +\sigma^{e}(t+\Delta t)\left[1+\frac{g_{1} \tau_{1}}{\Delta t}\left(1-e^{-\Delta t / \tau_{1}}\right)+\frac{g_{2} \tau_{2}}{\Delta t}\left(1-e^{-\Delta t / \tau_{2}}\right)\right] \\
& +c_{1}(t) e^{-\Delta t / \tau_{1}}+c_{2}(t) e^{-\Delta t / \tau_{2}}
\end{aligned}
$$


where $\sigma^{e}$ is defined as

$$
\sigma^{e}(t)=E_{f} E_{N}(t)
$$

where $E_{f}$ is the elastic fiber modulus of collagen. This allows for the viscoelastic COL fiber stress to be input into the UMAT as

$$
\begin{aligned}
& \sigma^{v}(t+\Delta t)=\frac{E_{f} E_{N}-E_{f} E_{n}}{\Delta t} {\left[g_{1} \tau_{1}\left(1-e^{-\Delta t / \tau_{1}}\right)+g_{2} \tau_{2}\left(1-e^{-\Delta t / \tau_{2}}\right)\right] } \\
&+E_{f} E_{N}+c_{1}(t) e^{-\Delta t / \tau_{1}}+c_{2}(t) e^{-\Delta t / \tau_{2}}
\end{aligned}
$$

where $E_{n}$ is the fiber Lagrangian strain in the reference configuration. Equation 34 can now be solved iteratively as

$$
\boldsymbol{\sigma}_{C O L}=\sum_{i=1}^{p} R H \sigma^{v}(\boldsymbol{N} \otimes \boldsymbol{N}) \mathrm{d} V,
$$

where $\mathrm{p}$ is the number of pyramid elements. The partial derivative of the elastic collagen stress with respect to fiber strain is

$$
\frac{\sigma^{e}}{\partial E_{N}}=E_{f}
$$

so that from Equation 40, the collagen elasticity tensor is calculated as

$$
\tilde{\mathbb{C}}_{C O L}=\int_{V} R H c E_{f}(\boldsymbol{N} \otimes \boldsymbol{N}) \otimes(\boldsymbol{N} \otimes \boldsymbol{N}) \mathrm{d} V
$$

which is solved iteratively similar to Equation 47

$$
\tilde{\mathbb{C}}_{C O L}=\sum_{i=1}^{p} R H c E_{f}(\boldsymbol{N} \otimes \boldsymbol{N}) \otimes(\boldsymbol{N} \otimes \boldsymbol{N}) \mathrm{d} V .
$$

\subsection{Anisotropic Distribution}

The anisotropic distribution used here was developed by Yamauchi [68]. A short description follows for clarity and continuity. 
A normal or Gaussian distribution function of COL fibers was investigated first

$$
f(x)=\frac{e^{-(x-\mu)^{2} / 2 \sigma^{2}}}{\sqrt{2 \pi \sigma^{2}}}
$$

where $\mu$ is the mean value of $x$ and $\sigma$ is the variance of $x$. We then normalize this function to satisfy the constraint that $\int f(x) \mathrm{d} x=1[58]$

$$
f^{\prime}(x)=\frac{e^{-(x-\mu)^{2} / 2 \sigma^{2}}}{\int e^{-(x-\mu)^{2} / 2 \sigma^{2}} \mathrm{~d} x} .
$$

The problem with this is that the distribution approaches zero on the periphery, which when implemented into our Abaqus model causes loss of convergence during the equilibrium step because there are not enough COL fibers to resist the GAG swelling stress. For this reason, it was proposed to develop a background isotropic distribution on top of this normalized Gaussian distribution, which is also seen in experimental data. A minimum isotropic distribution was determined by qPLM analysis of four tissue samples harvested from the same tissue source and incubated for four days in medium supplemented with fetal bovine serum. qPLM specimens were $0-1 \mathrm{~mm}$ from the surface. One assumption here is that the distribution is identical between D0 and D12-TGF- $\beta 1$. Yamauchi [68] then accomplished the desired distribution with the following:

1. Calculate the normalized Gaussian distribution as explained above (discretized into a specified number of elements).

2. Step through each element of the distribution that is below the minimum isotropic requirement and determine the difference between each and the minimum isotropic value.

3. Add the value calculated in the previous step to the original distribution. The distribution will not integrate to 1 in this step. 
4. Calculate the volume fraction of fibers added to the distribution and use this value to scale the distribution so that it integrates to 1 again. Some values will again dip below the minimum value.

5. Iterate over steps $2-4$ until all elements have a value above the minimum required and the function integrates to 1 .

\subsection{SWELling AND CALCUlATiNG STRAin}

In order to compare results to experimental values, it is necessary to begin with a stress free reference configuration. To accomplish this, an equilibrium step is run before loading in Abaqus. This creates some complications as it is necessary to know the displacement after the equilibrium step in order to appropriately displace the model to the prescribed strain. Previous models only ran one simulation at a time, so it was possible to first run the equilibrium step, look at the results, and complete the appropriate displacement in subsequent simulations. Since the current simplex optimization routine runs several models sequentially without the possibility of stopping, viewing results, and changing boundary conditions, a more automated process was created.

Using a Python script, first an equilibrium only simulation is completed. The output database file is then opened (which stores all specified solutions including stress, displacement, and STATEVs) and the displacement for the top nodes is extracted. This displacement is added to the original height and multiplied by $5 \%$ in order to calculate the necessary displacement to achieve 5\% strain. Since Abaqus defines all boundary conditions from the initial reference configuration, this displacement must be added to the equilibrium displacement in order to achieve the necessary strain (i.e. effectively make the configuration after equilibrium the new reference configuration). The script then opens the model database for the complete simulation (i.e. the simulation which includes equilibrium, loading, and relaxation steps), and updates the displacement boundary condition to be this calculated num- 
ber (See Figure 4 for a graphical representation). The script then starts the complete simulation and continues the optimization.

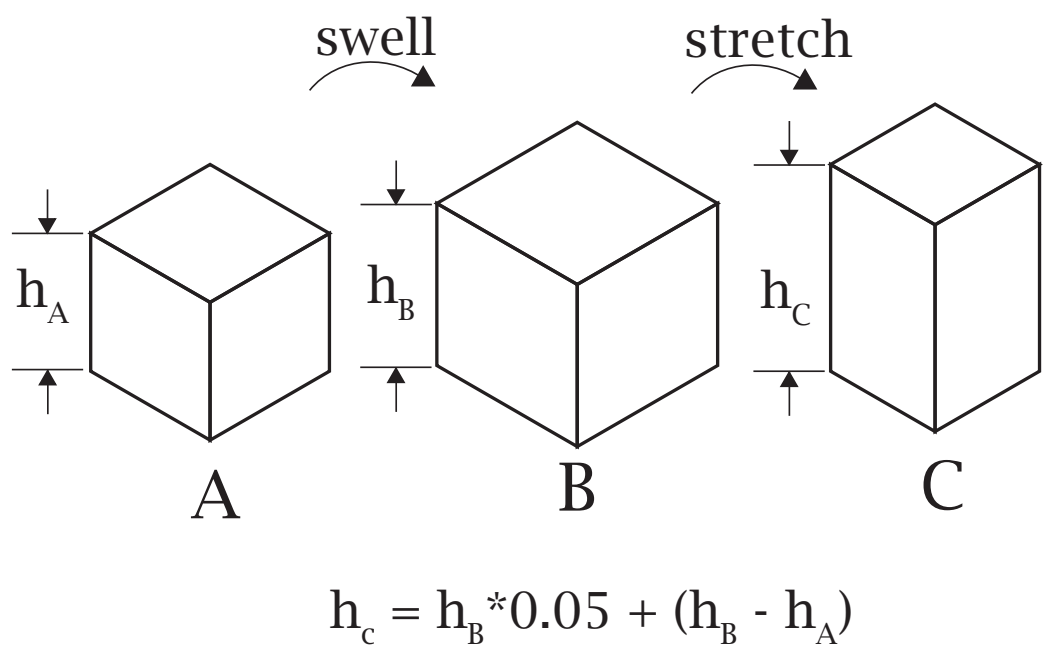

Figure 4: Experimental reference configuration and Abaqus. Abaqus specifies displacement boundary conditions from the initial reference configuration (A). If we were to input a displacement boundary condition of $5 \%$ strain, it would only be $h_{A} * 0.05$, which would not correctly model the experimental procedure. The experimental procedure is $5 \%$ strain of the stress free reference configuration (B). To account for this we must find the height after swelling $\left(h_{B}\right)$, calculate $5 \%$ strain of this height, and add the change after swelling $\left(h_{B}-h_{A}\right)$ to determine the displacement boundary condition for the loading step (stretch).

\subsection{Abaqus - Uniaxial Tension}

The experimental test (Section 4.1) consisted of a dogbone shaped tab pulled in tension (Figure 5). The cycles consists of a $40 \mathrm{~s}$ ramp loading to $5 \%$ strain and a 5000 s relaxation step. Some tare strain was recorded during experimental testing, often on the same order of magnitude as total strain. It was decided that this tare strain was not actually being transmitted to the specimen, and was most likely due to specimen sag and jaw-slippage. Models were run with this tare strain neglected. The experimental tare stress, however, was included because there was no rigorous method to determine how much of the stress was actually being felt by the specimen. 


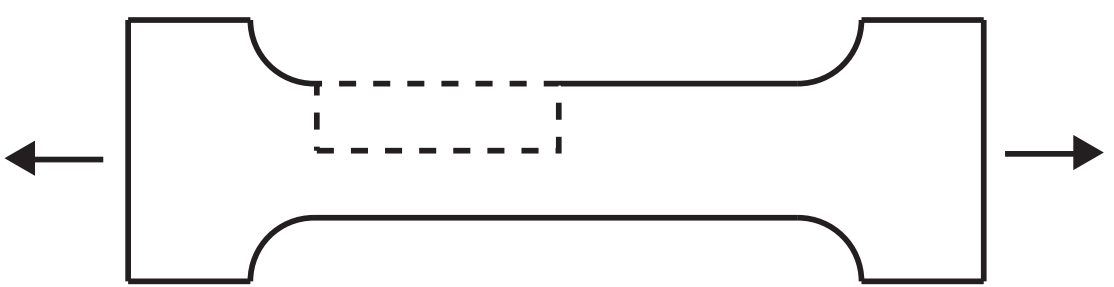

Figure 5: Graphical representation of experimental procedure. Axisymmetric considerations show that we can reduce this model to the dashed rectangle.

This model used a linear 3D stress solid element (C3D8). The element was $1 \mathrm{~mm}$ x $1 \mathrm{~mm}$ x $1 \mathrm{~mm}$. Boundary conditions were considered "box in a corner" where all faces touching a wall were fixed using Abaqus' displacement boundary condition and applying a 0 displacement. After the equilibrium step, the top face of the element was displaced according to the procedure described above. All boundary conditions were propagated through the relaxation step. The model and boundary conditions used are in Figure 6.

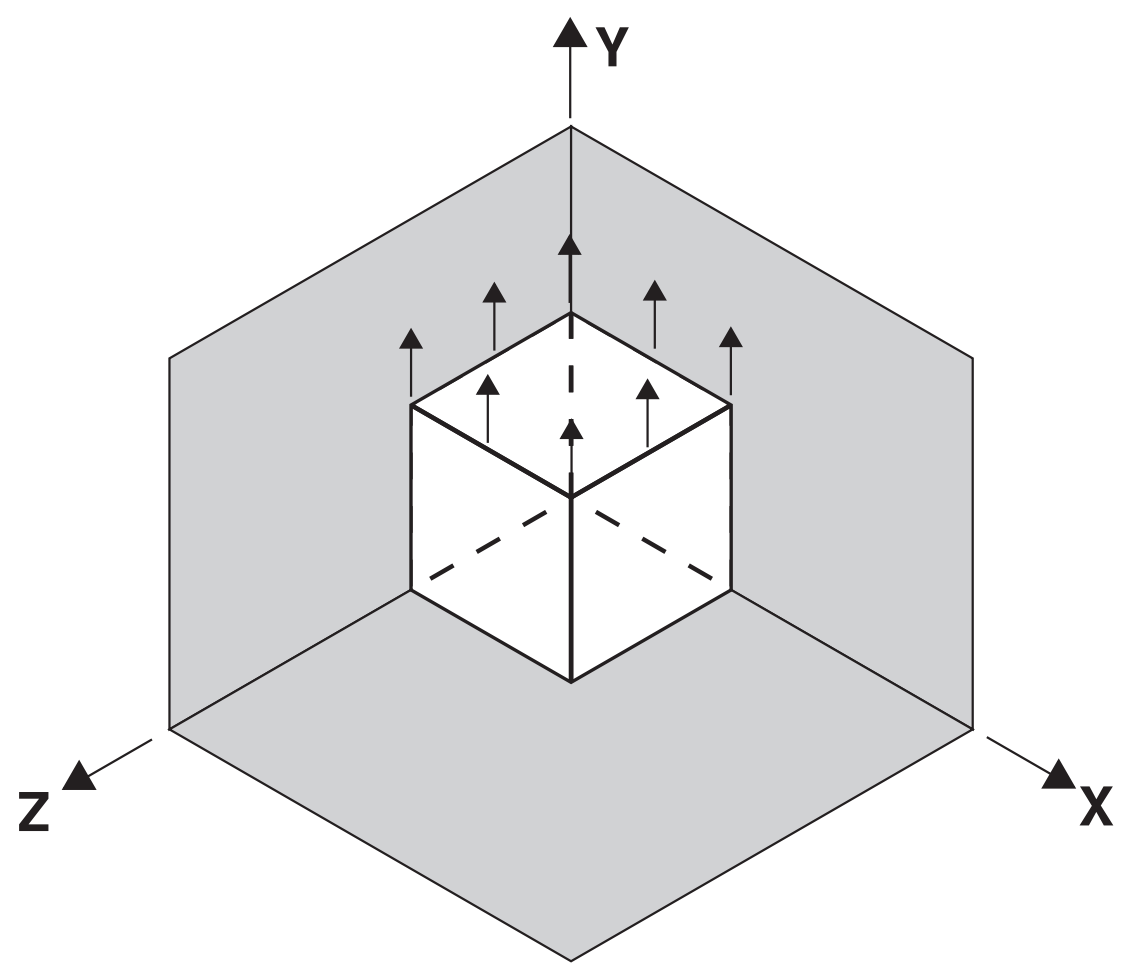

Figure 6: The model used in an Abaqus simulation of uniaxial tension. The boundary conditions were considered "box in a corner." The faces against the shaded walls were fixed, while the other faces were free. The positive-y face was then displaced to $5 \%$ relative to the swelling configuration. 
A mesh convergence study (Appendix B) showed that results from a single element were identical to a full model. Finite element analysis (FEA) was still used because it was necessary to validate the constitutive relationship within the UMAT so that future models that necessitate FEA (i.e. models including permeability or perhaps geometric complexity) will be able to use this UMAT with confidence.

\subsection{SimpleX}

In order to fit the model to experimental results, a simplex optimization method was validated in MATLAB [76] and then coded into a Python script.

\subsubsection{Simplex VAlidation}

The simplex optimization method was developed by Nelder and Mead [82] and has been updated and validated thoroughly. A simple explanation and visual validation will be presented here for clarity.

Given an arbitrary function $f(x, y)$ and the vertices of an initial simplex $v=$ $\left[x_{1}, y_{1} ; x_{2}, y_{2} ; x_{3}, y_{3}\right]$, the local minima is found using the steps following Figure 7 .

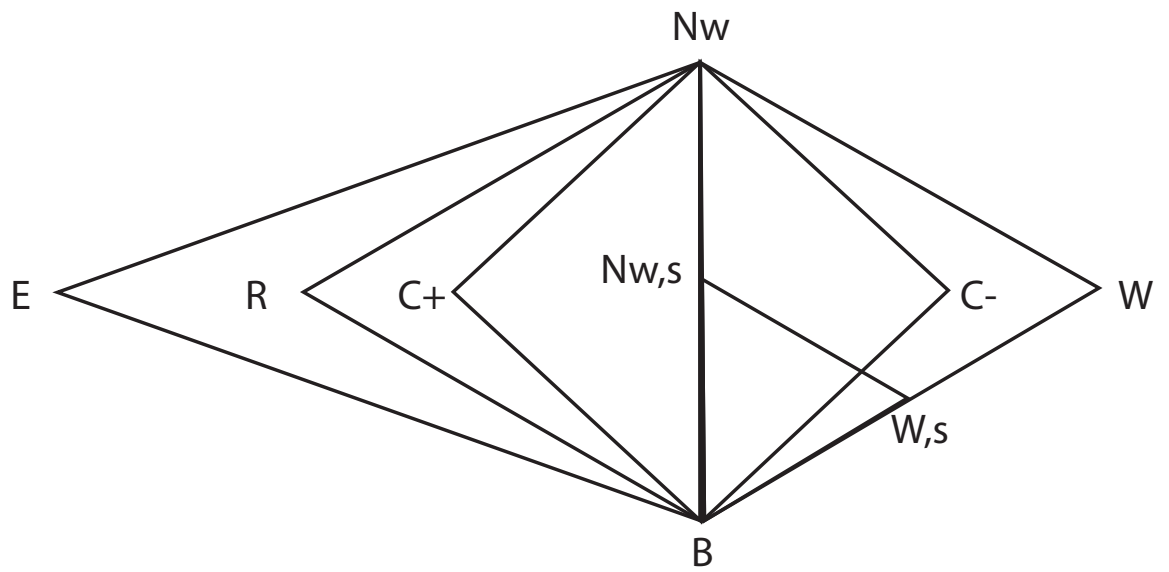

Figure 7: The movements of the simplex during minimization. Simplex B-Nw-W is the initial simplex. B corresponds to the best solution, $\mathrm{W}$ the worst solution, $\mathrm{Nw}$ the next worst solution. The remaining vertices each correspond to one possible step. $\mathrm{R}$ would be a simple reflection, $\mathrm{E}$ an expansion, $\mathrm{C}+$ positive contraction, $\mathrm{C}$ - negative contraction, $\mathrm{Nw}, \mathrm{s}$ and $\mathrm{W}, \mathrm{s}$ correspond to shrinking. The process to decide which motion to take and how to calculate the new vertex is shown below. 


\section{Sort and Rank}

The first step is to evaluate the objective function at each of the vertices and then sort the results (along with their corresponding vertices), assigning the naming convention "B" for the best result (lowest), "W" for the worst result (highest), and "Nw" for the next worst result (middle).

2. $\bar{v}$

Next, calculate the centroid of the better two vertices ( $\mathrm{B}$ and $\mathrm{Nw}$ )

$$
\begin{aligned}
& \bar{v}(1)=\frac{x_{B}+x_{N w}}{2}, \\
& \bar{v}(2)=\frac{y_{B}+y_{N w}}{2} .
\end{aligned}
$$

\section{Next Vertex}

Now begins the evaluation of where the next vertex will be. To begin, calculate the vertex of just a reflected simplex.

$$
v_{R}=(1+\alpha) \bar{v}-\alpha v_{W}
$$

where $\alpha$ is the reflection coefficient (typically $\alpha=1$ ) and $v_{W}$ corresponds to the vertex of the worst function evaluation. The function is then evaluated at this vertex $\left(f_{R}=f\left(v_{R}\right)\right.$, let all other function evaluations follow this naming convention).

(a) Negative Check

Since a negative parameter is physically impossible and would cause Abaqus to diverge, a check must take place before the next simulation runs. If any of the parameters are found to be negative, a positive contraction is performed until that value is no longer negative.

(b) If $f_{R}>f_{B}$ and $f_{R}<f_{N w}$ 
The simplex is merely reflected. In vanilla Nelder-Mead (without expansion, contraction, or shrinking) this is the only step. $v_{W}$ becomes $v_{R}$ and $f_{W}$ becomes $f_{R}$.

(c) If $f_{R}<f_{B}$

The reflection is along the right path and expansion is evaluated to hopefully speed up the process. First, calculate the expansion vertex

$$
v_{E}=(1+\gamma) \bar{v}-\gamma v_{W}
$$

where $\gamma$ is the expansion coefficient (typically $\gamma=2$ ).

i. Negative Check

Again, a check for negative parameters must take place. If any of the parameters are negative, they are replaced with the respective parameter from the reflection vertex.

ii. If $f_{E}<f_{R}$

We are indeed traveling along the correct path and can complete an expansion. $v_{W}$ becomes $v_{E}$ and $f_{W}$ becomes $f_{E}$.

iii. Else

Expansion will not be beneficial, and only a reflection will be performed. $v_{W}$ becomes $v_{R}$ and $f_{W}$ becomes $f_{R}$.

(d) If $f_{N w}<f_{R}<f_{W}$

A positive contraction will be evaluated. Calculate the positive contraction vertex

$$
v_{C}=(1+\beta) \bar{v}-\beta v_{W}
$$

where $\beta$ is the contraction coefficient (typically 0.5 ).

i. If $f_{C}<f_{R}$

Complete a positive contraction. $v_{W}$ becomes $v_{C}$ and $f_{W}$ becomes $f_{C}$. 
(e) If $f_{R}>f_{W}$

A negative contraction will be evaluated. Calculate the negative contraction vertex

$$
v_{C}=(1-\beta) \bar{v}+\beta v_{W}
$$

Again, $\beta$ is the contraction coefficient (typically 0.5 ).

i. If $f_{C}<f_{W}$

Complete a negative contraction. $v_{W}$ becomes $v_{C}$ and $f_{W}$ becomes $f_{C}$.

(f) Re-sort or Shrink and Restart

If one of the steps above was completed, the new function evaluations and corresponding vertices are re-sorted (and re-labeled). The distance between the best and the worst vertex is evaluated. If this distance is within a specified tolerance, the local minima has been found. If it is not, the process begins again at Step 2.

If $v_{R}$ did not satisfy any of the above criteria, then the simplex is shrunk. All but the best vertex are changed according to

$$
v=v_{B}+\rho\left(v-v_{B}\right)
$$

and $f$ is re-evaluated at these vertices. After shrinking, the function and vertices are sorted and the process begins again at Step 2.

This method is easily scaled up to higher variable methods with minor changes. The 2-variable simplex code was applied to the Rosenbrock function [77], and the 3-variable code to Fletcher and Powell's helical valley [78], both common tests of optimization functions. 


\subsubsection{Validation Results}

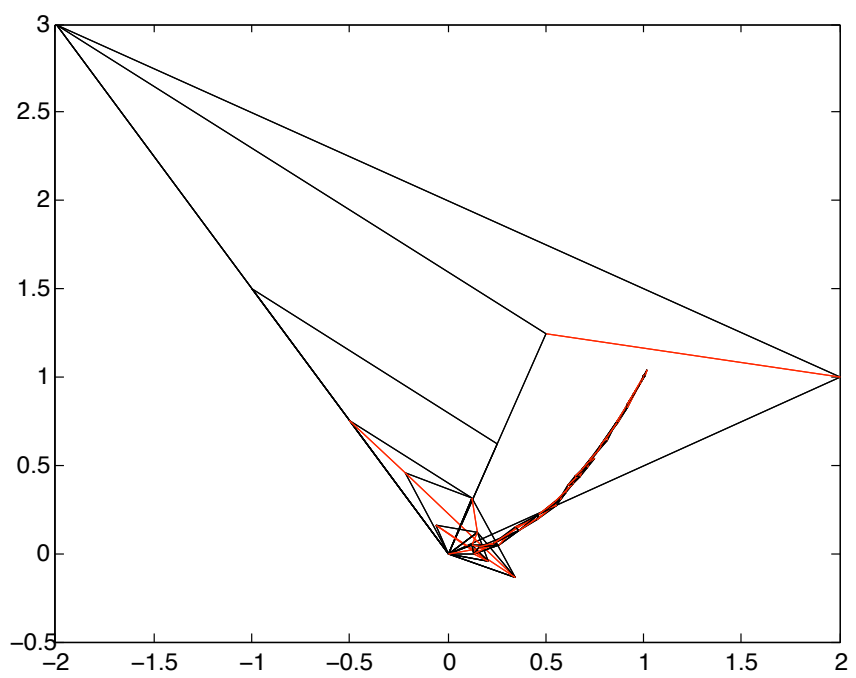

Figure 8: Visualization of the simplex movements applied to the 2-variable Rosenbrock function $f(x, y)=(1-x)^{2}+100\left(y-x^{2}\right)^{2}$ as it finds the minimum. The minimum was found at $[1,1]$ of $\mathrm{f}=0$. The black lines are the simplexes, and the red line shows which vertex moved during each step.

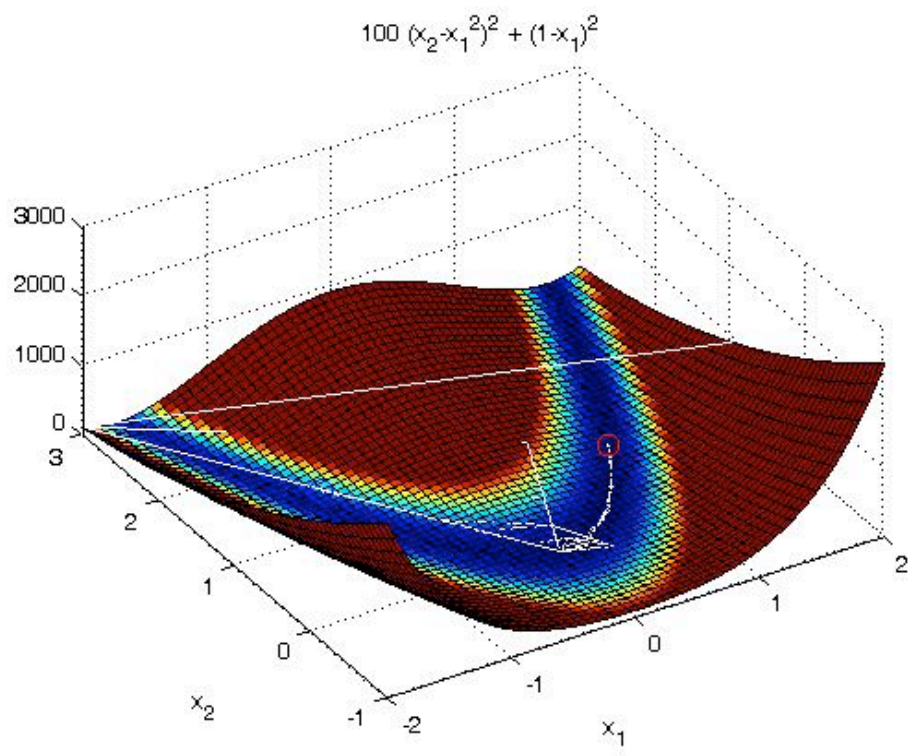

Figure 9: Visualization of the same simplex movements (Figure 8) across the 3D surface of the Rosenbrock function. The white lines show the simplexes, and the red circle is the result. 


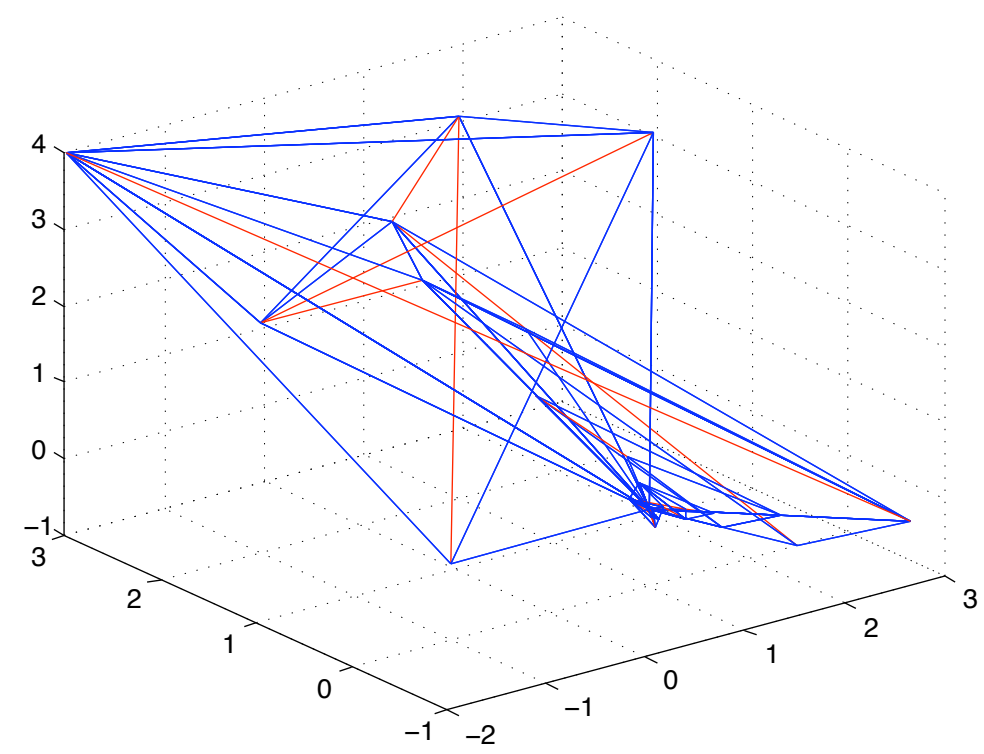

Figure 10: Visualization of the simplex movements applied to the 3-variable Fletcher-Powell helical valley $f(x, y, z)=100[z-10 \theta(x, y)]^{2}+\left[\sqrt{x^{2}+y^{2}}-1\right]^{2}+z^{2}$ where $2 \pi \theta(x, y)=\arctan (y / x), x>0$ or $2 \pi \theta(x, y)=\pi+\arctan (y / x), x<0$. The minimum was found at $[1,0,0]$ of $\mathrm{f}=0$. The blue lines show the simplexes and the red line again shows which vertex moved during each step.

\subsubsection{Simplex Applichtion}

For this study, the objective function being minimized is the sum of the squared errors

$$
\mathcal{S}_{\text {err }}=\sum_{i=1}^{m}\left(y_{i}-f_{i}\right)^{2}
$$

where $m$ is the number of observations, $y_{i}$ are the experimental values, and $f_{i}$ are the modeled values. In order to simplify the process, the experimental data was decimated with some bias towards initial relaxation. Experimental data was output at 907 points, this decimation reduced the data to 51 points. Figure 11 shows an example of the decimation used. 


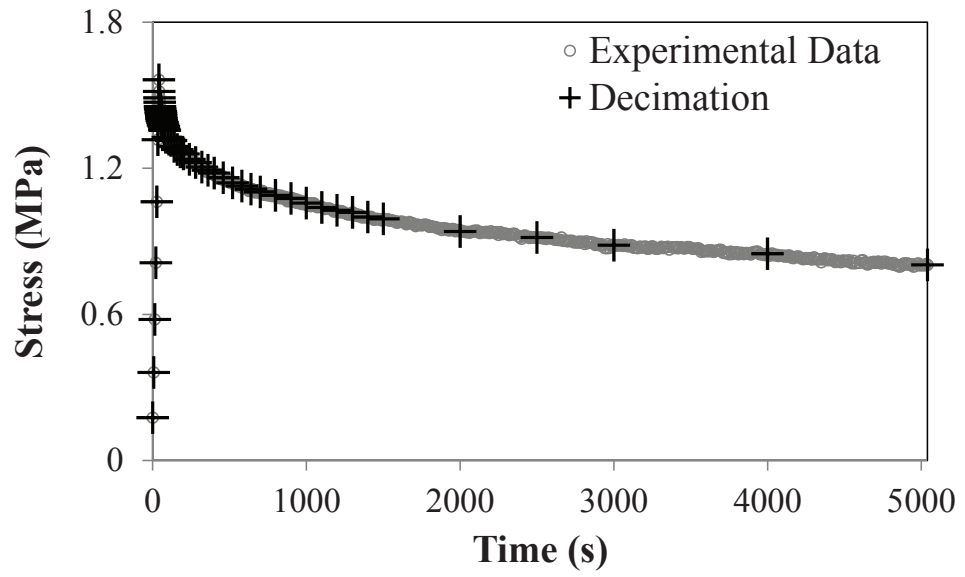

Figure 11: Decimation used in optimization. Points were picked to bias towards initial relaxation. 51 points were compared between experimental data and model data.

The inputs to this function are the model parameters (i.e. $g_{1}, g_{2}, \tau_{1}$, and $\tau_{2}$ ). The simplex optimization method must start with an initial set of inputs (collectively called vertices) which are evaluated and create the first polyhedron. The initial number of vertices is always one more than the parameters being optimized, which in this case is four $\left(g_{1}, g_{2}, \tau_{1}\right.$, and $\left.\tau_{2}\right)$, so five initial vertices started the simplex. This resulted in a four-dimensional surface in a five-dimensional space. Some manual optimization using brute force trial-and-error was performed to give the simplex optimization method an appropriate starting point (i.e. starting vertices resulted in solutions with similar relaxation profiles and same order of magnitude maximum stress as experimental data). The starting vertices for the average tissue samples are shown in Table 2. 
Table 2: Starting vertices of the average tissue samples for the simplex optimization method.

\begin{tabular}{|c|c|c|c|c|}
\hline Tissue & $g_{1}$ & $\tau_{1}$ & $g_{2}$ & $\tau_{2}$ \\
\hline \multirow{5}{*}{ D0-S } & 1.051 & 700 & 0.4 & 30 \\
\hline & 1.055 & 600 & 0.25 & 28 \\
\hline & 1.04 & 850 & 0.35 & 20 \\
\hline & 1.06 & 750 & 0.3 & 15 \\
\hline & 1.09 & 800 & 0.2 & 25 \\
\hline \multirow{5}{*}{ D0-M } & 1.049 & 1000 & 0.01 & 25 \\
\hline & 1.075 & 1050 & 0.015 & 35 \\
\hline & 1.08 & 1150 & 0.025 & 40 \\
\hline & 1.1 & 1100 & 0.02 & 30 \\
\hline & 1.099 & 1073 & 0.0176 & 20 \\
\hline \multirow{5}{*}{ D12-S } & 0.6 & 1000 & 0.25 & 30 \\
\hline & 0.55 & 950 & 0.2 & 25 \\
\hline & 0.5 & 900 & 0.15 & 20 \\
\hline & 0.45 & 844 & 0.119 & 10 \\
\hline & 0.4 & 800 & 0.1 & 15 \\
\hline \multirow{5}{*}{ D12-M } & 0.45 & 844 & 0.119 & 10 \\
\hline & 0.4 & 800 & 0.1 & 15 \\
\hline & 0.5 & 900 & 0.15 & 20 \\
\hline & 0.55 & 950 & 0.2 & 25 \\
\hline & 0.6 & 1000 & 0.25 & 30 \\
\hline
\end{tabular}

\subsection{Data SETS}

Four groups were considered - untreated explants from the middle and superficial layers and D12-TGF- $\beta 1$ explants from the middle and superficial layers. Initial simulations looked at the average of each tissue group. Subsequent simulations looked at each tissue sample from those groups individually in order to complete 
two-factor analysis of variance (ANOVA) and post-hoc Tukey testing. Table 3 lists the number of samples used from each group.

Table 3: Number of samples from each tissue group. D0 are untreated explants. D12 TGF- $\beta 1$ are explants treated in transforming growth factor $-\beta 1$ for 12 days. S are explants from the superficial layer and $\mathrm{M}$ from the middle layer.

\begin{tabular}{|l|c|}
\hline Tissue Group & No. of Samples \\
\hline D0 - S & 6 \\
\hline D0 - M & 7 \\
\hline D12 - TGF- $\beta 1-$ S & 5 \\
\hline D12 - TGF- $\beta 1-$ M & 4 \\
\hline
\end{tabular}

\subsection{Convergence}

Some convergence issues were encountered during modeling. Loss of convergence occurred in the displacement residuals during relaxation, with the error being displacement correction too large compared to maximum displacement. The Abaqus help files suggests that this error may be incorrectly encountered when the displacement during a step is effectively zero (which is the case during relaxation). Also, Vena [79] suggests that as long as the force equilibrium equations are converging, it is appropriate to ignore the displacement residuals. The displacement equations can be effectively ignored by setting the ratio of allowable displacement correction to maximum displacement $\left(C^{\alpha}\right)$ to 100 during the relaxation step. This is accomplished with the addition of the keyword

*Controls, parameters=field, field=displacement , 100., , , , ,

This greatly increased convergence, but care was taken in validating results since Abaqus could possibly accept incorrect results. In particular, final results of the simplex optimization script were rerun with default solution controls to insure that the same results were calculated. 


\subsection{Computation}

Each individual tissue completed approximately 50-70 iterations within the simplex optimization script with a tolerance set to $1 \mathrm{E}-4$. Tolerance was defined as the difference between the sum of squared errors of the best vertex and the worst vertex. This tolerance seemed to be the best balance between computation time and precision. This many iterations took approximately 24-36 hours for each simulation on Dell OptiPlex GX620 with a Pentium D processor, 4G RAM, running Windows 7 and Abaqus 6.10-2 (Abaqus/Standard implicit analysis).

\subsection{Statistical Analysis}

In order to determine significant difference between groups, two-factor ANOVA and post-hoc Tukey testing was performed on each parameter (i.e. $E_{f}, g_{1}, g_{2}, \tau_{1}$, and $\tau_{2}$ ) using location (S vs. M) and treament (untreated vs. treated in TGF- $\beta 1$ ) factors. 


\section{Results}

Results for the anisotropic QLV model are shown below. A permeability study (Appendix C) showed that when comparing models with and without permeability, the relaxation curves are identical, the only difference being a 5\% drop in maximum stress for the average D0-S specimen and a $2.8 \%$ drop in maximum stress for the average D0-M specimen when not including permeability.

Table 4: Viscoelastic parameters for the QLV model for different average tissue groups. $E_{f}$ and $\mu$ were determined from elastic tensile simulations of each tissue group, using the

simplex method to fit to the equilibrium Young's modulus. $g_{1}, \tau_{1}, g_{2}$, and $\tau_{2}$ were determined using the simplex method to minimize the sum of squared errors (Equation 60) between model results and experimental data.

\begin{tabular}{|c|c|c|c|c|c|c|}
\hline Tissue & $\begin{array}{c}E_{f} \\
(\mathrm{MPa})\end{array}$ & $\begin{array}{c}\mu \\
(\mathrm{MPa})\end{array}$ & $\begin{array}{c}g_{1} \\
(-)\end{array}$ & $\begin{array}{c}\tau_{1} \\
(\mathrm{~s})\end{array}$ & $\begin{array}{c}g_{2} \\
(-)\end{array}$ & $\begin{array}{c}\tau_{2} \\
(\mathrm{~s})\end{array}$ \\
\hline D0-S & 337 & 0.001 & 0.79 & 1037 & 1.39 & 23 \\
\hline D12-TGF- $\beta 1-\mathrm{S}$ & 828 & 0.001 & 0.51 & 850 & 0.19 & 16 \\
\hline D0-M & 1227 & 0.001 & 0.90 & 1146 & 0.26 & 142 \\
\hline D12-TGF- $\beta 1-\mathrm{M}$ & 2489 & 0.001 & 0.45 & 871 & 0.15 & 17 \\
\hline
\end{tabular}

Table 5: Overall viscoelastic response of each average tissue source and model. $R_{\text {exp }}$ is the ratio of equilibrium stress to maximum stress and $\tau_{\text {exp }}$ is the time constant of each tissue set (calculated as the time required for the stress to relax 63.2\%). Percent difference is the difference between that tissue's calculated $R_{\exp }$ or $\tau_{\exp }$ and the experimental values.

\begin{tabular}{|c|c|c|c|c|}
\hline Tissue & $\begin{array}{c}R_{\exp } \\
(-)\end{array}$ & $\begin{array}{c}\% \\
\text { Diff. }\end{array}$ & $\begin{array}{c}\tau_{\exp } \\
(\mathrm{s})\end{array}$ & $\begin{array}{c}\% \\
\text { Diff. }\end{array}$ \\
\hline D0-S (Exp.) & 0.48 & - & 460 & - \\
\hline D0-S & 0.497 & 3.2 & 580 & 26.1 \\
\hline D0-M (Exp.) & 0.51 & - & 844 & - \\
\hline D0-M & 0.56 & 9.4 & 1000 & 18.4 \\
\hline D12-TGF- $\beta 1-S$ (Exp.) & 0.63 & - & 344 & - \\
\hline D12-TGF- $\beta 1-S$ & 0.68 & 8.4 & 822 & 139 \\
\hline D12-TGF- $\beta 1-M$ (Exp.) & 0.70 & - & 613 & - \\
\hline D12-TGF- $\beta 1-M$ & 0.73 & 4.9 & 840 & 36 \\
\hline
\end{tabular}




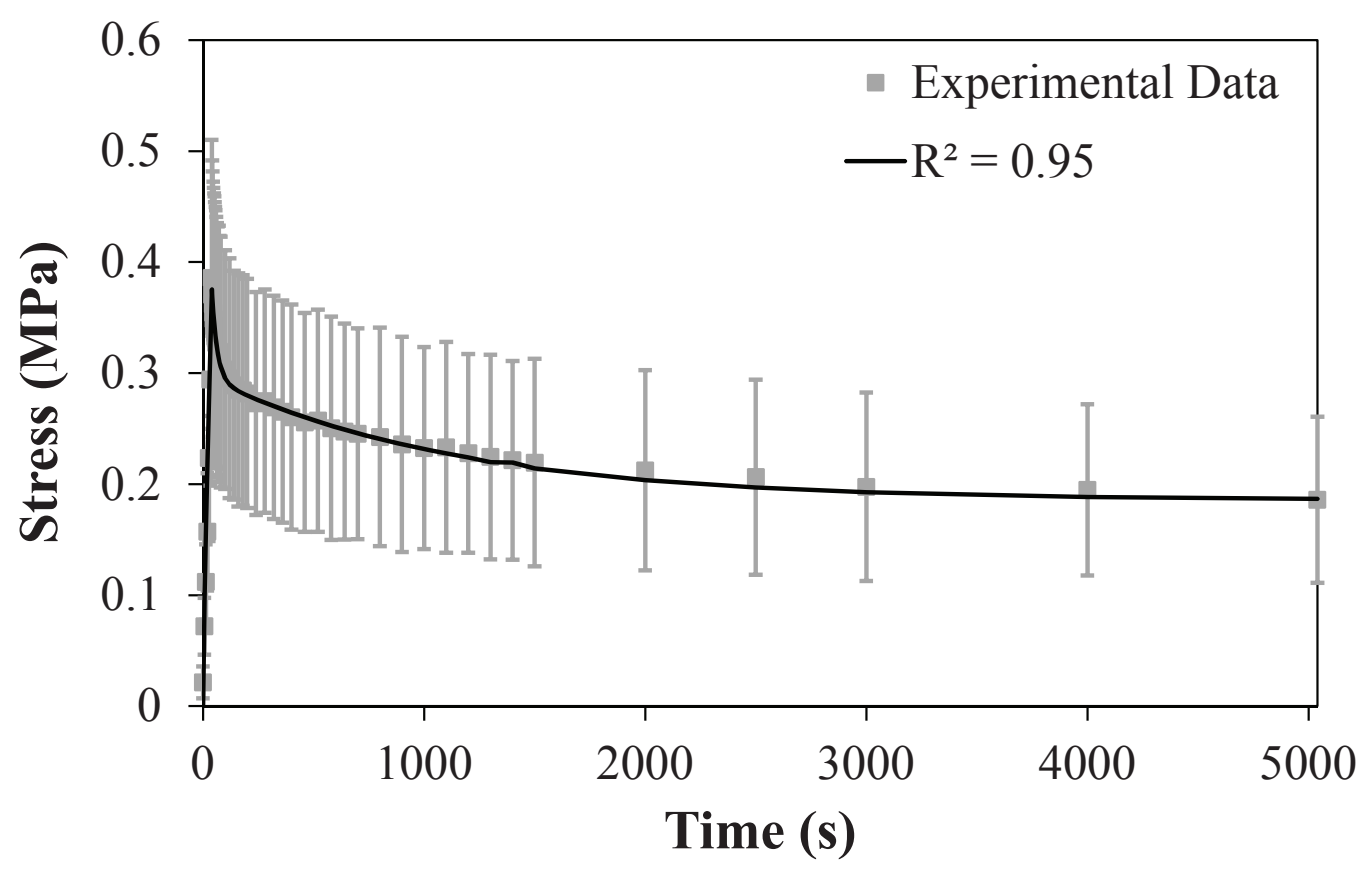

Figure 12: Fit of average untreated superficial layer specimens.

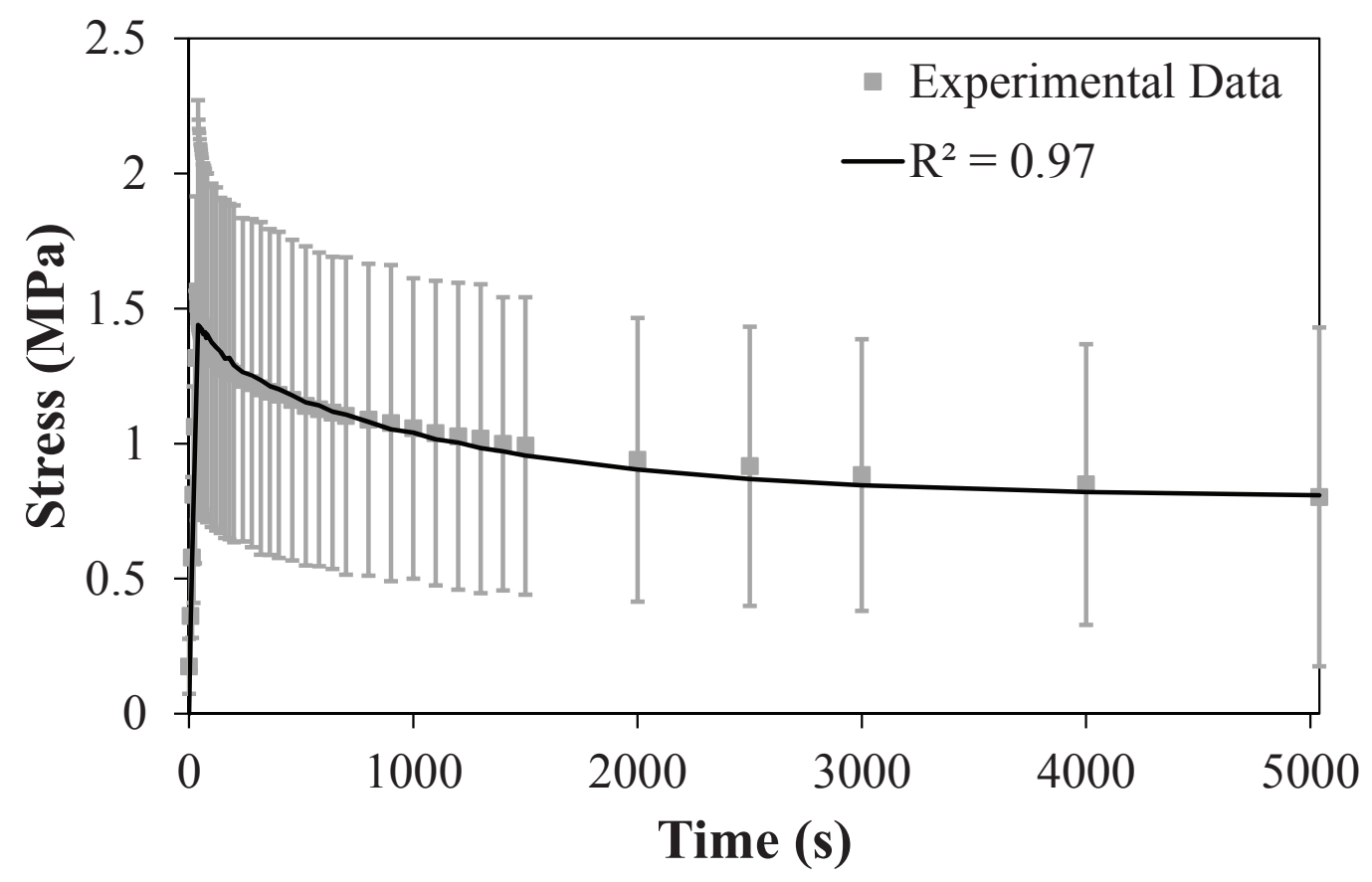

Figure 13: Fit of average untreated middle layer specimens. 


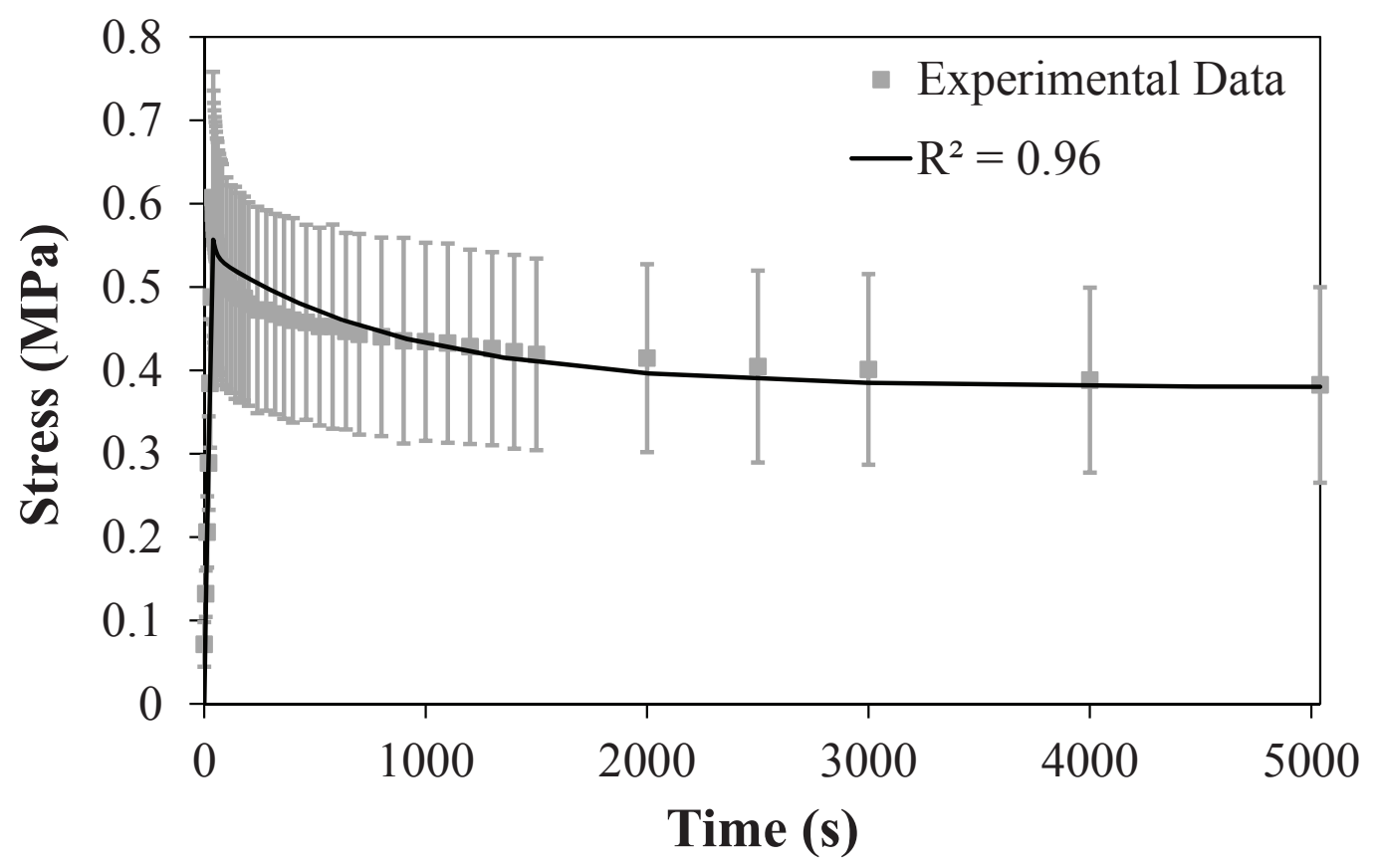

Figure 14: Fit of average superficial layer specimens treated for 12 days in TGF- $\beta 1$.

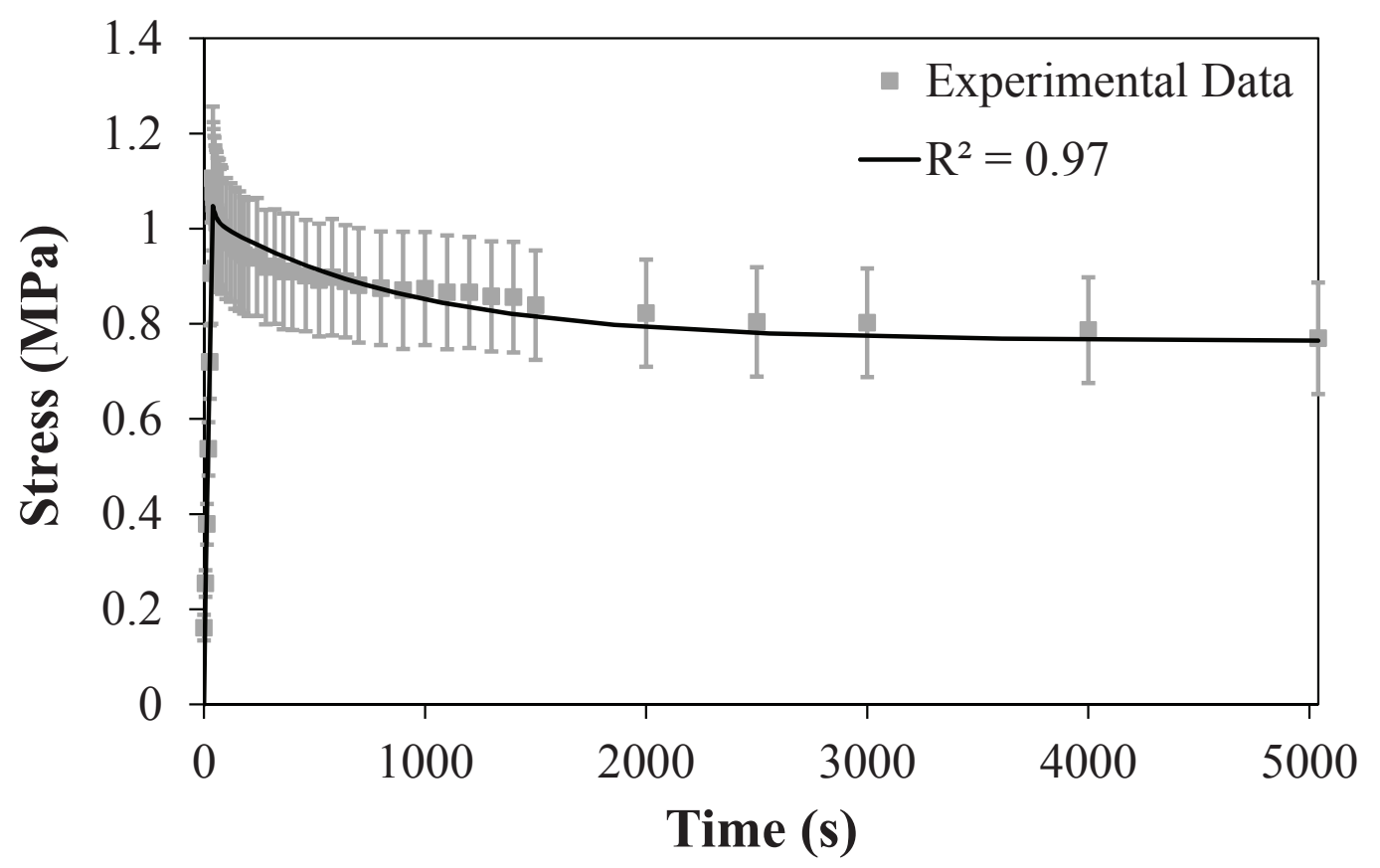

Figure 15: Fit of average middle layer specimens treated for 12 days in TGF- $\beta 1$. 
Table 6: Average elastic parameters for COL from from each individual specimen. $E_{f}$ was determined from elastic tensile simulations of each tissue group, using the simplex method to fit to the equilibrium Young's modulus. These results are also plotted in Figure 16. $E_{f}$ listed as mean \pm one standard deviation. $\mu$ was kept fixed so its standard deviation was not calculated.

\begin{tabular}{|c|c|c|}
\hline Tissue & $\begin{array}{c}E_{f} \\
(\mathrm{MPa})\end{array}$ & $\begin{array}{c}\mu \\
(\mathrm{MPa})\end{array}$ \\
\hline D0-S & $359 \pm 176$ & 0.001 \\
\hline D12-TGF- $\beta 1-\mathrm{S}$ & $1571 \pm 1271$ & 0.001 \\
\hline D0-M & $1012 \pm 491$ & 0.001 \\
\hline D12-TGF- $\beta 1-\mathrm{M}$ & $2706 \pm 1021$ & 0.001 \\
\hline
\end{tabular}

Table 7: Average viscoelastic parameters for the QLV model from from each individual specimen. $g_{1}, \tau_{1}, g_{2}$, and $\tau_{2}$ were determined using the simplex method to minimize the sum of squared errors (Equation 60) between model results and experimental data. These results are also plotted in Figure 17 - 20. All values listed as mean \pm one standard deviation.

\begin{tabular}{|c|c|c|c|c|}
\hline Tissue & $\begin{array}{c}g_{1} \\
(-)\end{array}$ & $\begin{array}{c}\tau_{1} \\
(\mathrm{~s})\end{array}$ & $\begin{array}{c}g_{2} \\
(-)\end{array}$ & $\begin{array}{c}\tau_{2} \\
(\mathrm{~s})\end{array}$ \\
\hline D0-S & $0.95 \pm 0.15$ & $1968 \pm 1861$ & $0.91 \pm 1.03$ & $50 \pm 34$ \\
\hline D12-TGF- $\beta 1-\mathrm{S}$ & $0.38 \pm 0.08$ & $1337 \pm 569$ & $0.44 \pm 0.18$ & $47 \pm 29$ \\
\hline D0-M & $1.13 \pm 0.19$ & $2385 \pm 697$ & $1.01 \pm 0.60$ & $48 \pm 24$ \\
\hline D12-TGF- $\beta 1-\mathrm{M}$ & $0.65 \pm 0.31$ & $2217 \pm 2890$ & $0.18 \pm 0.24$ & $107 \pm 190$ \\
\hline
\end{tabular}



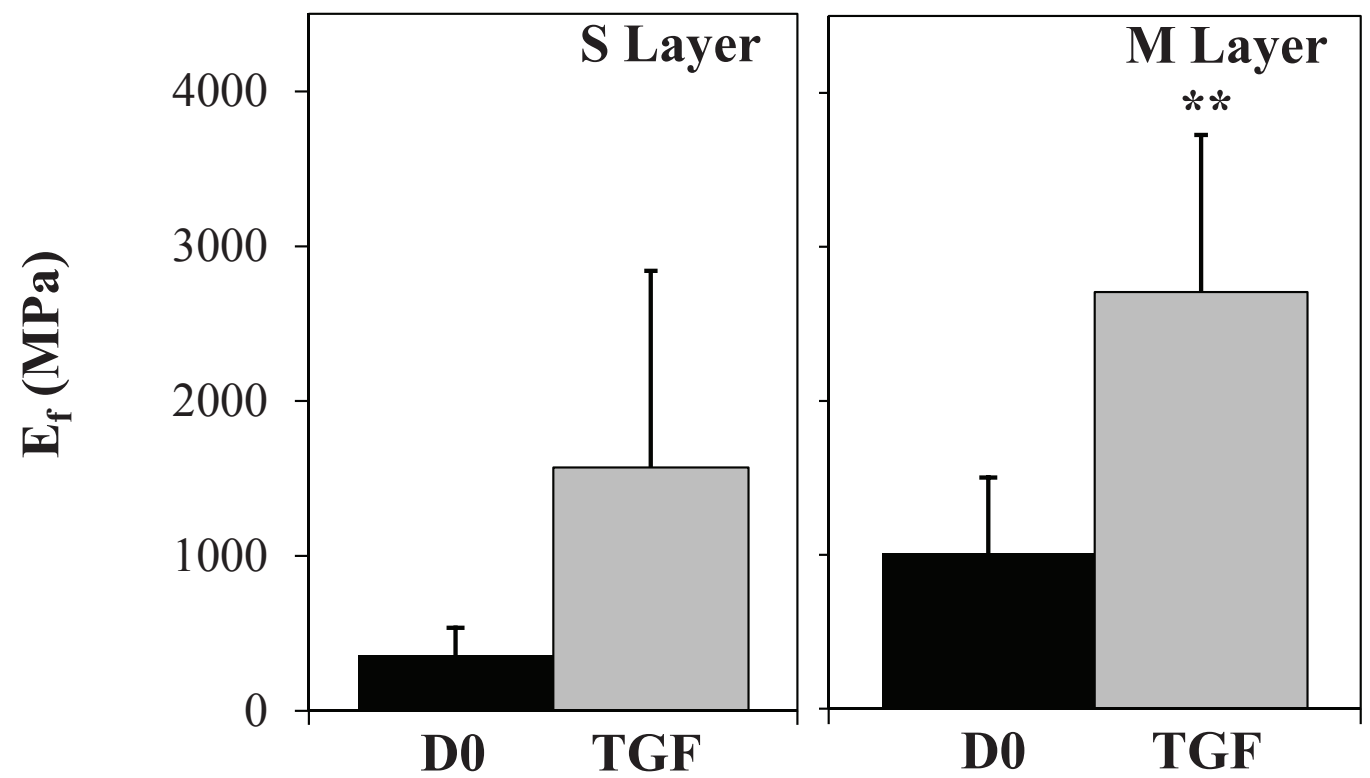

Figure 16: Effect of TGF- $\beta 1$ on D0-S and D0-M elastic collagen fiber modulus. ** corresponds to a significant difference from D0-M (two-factor ANOVA, post-hoc Tukey test, $p<0.05)$. There is also a significant difference between D0-S and D12-TGF- $\beta 1-\mathrm{M}$ $(p=0.001)$.
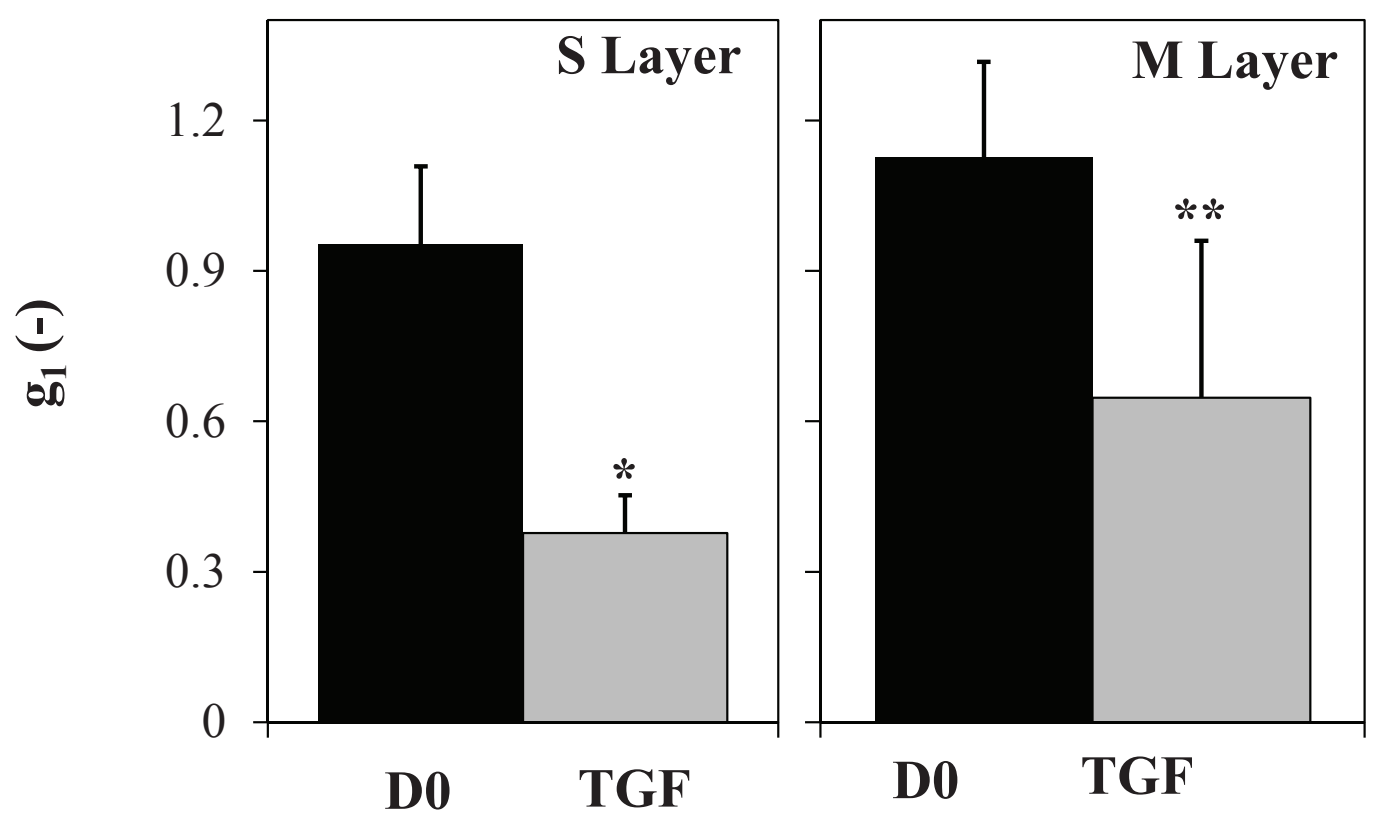

Figure 17: Effect of TGF- $\beta 1$ on D0-S and D0-M viscoelastic parameter $g_{1}$ (the long term relaxation coefficient). ${ }^{*}$ corresponds to a significant difference from D0-S $(p<0.005)$ and ** a significant difference from D0-M $(p<0.001)$ (two-factor ANOVA, post-hoc Tukey test). There is also a significant difference between D0-M and D12-TGF- $\beta 1-\mathrm{S}(p<0.001)$. 


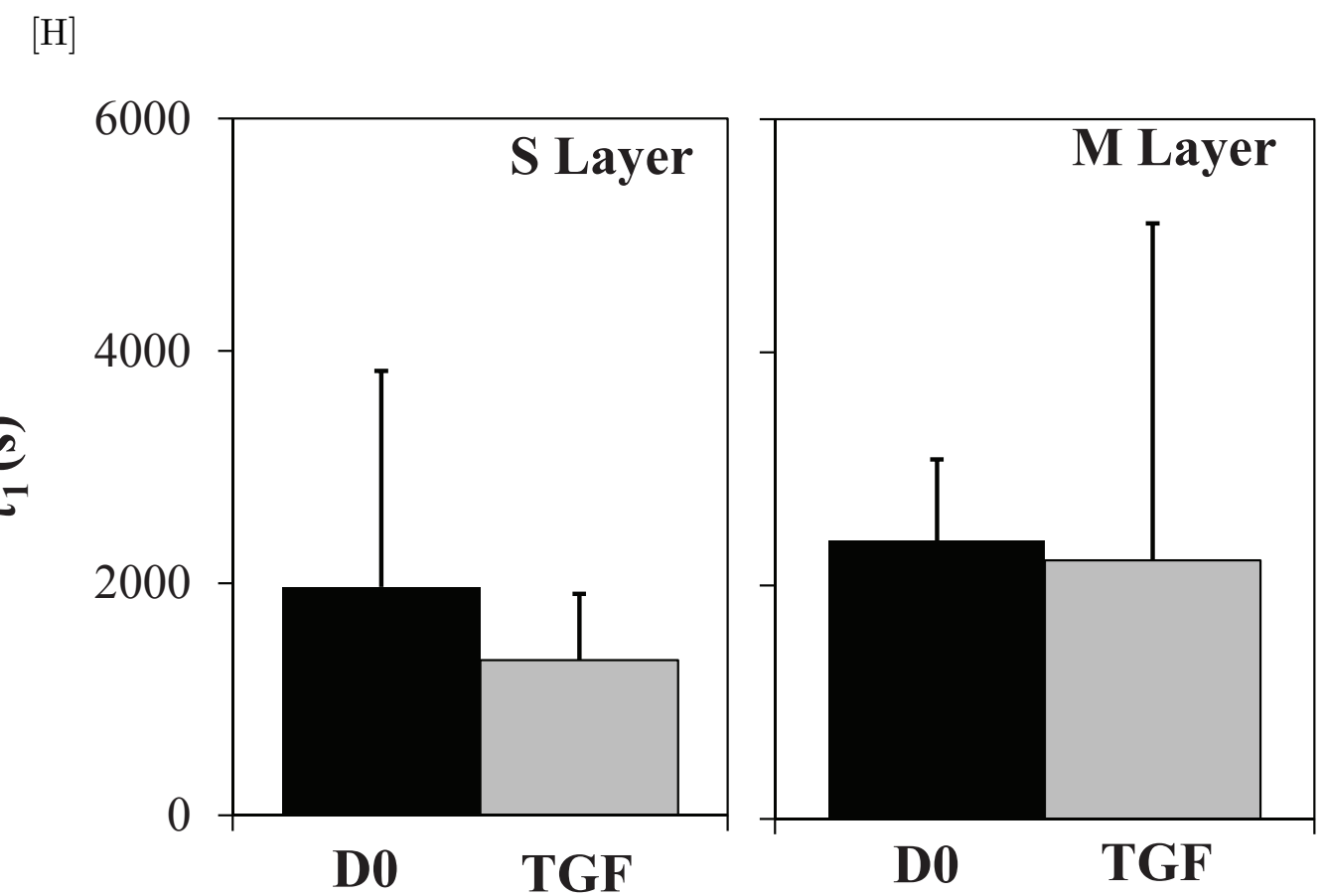

Figure 18: Effect of TGF- $\beta 1$ on D0-S and D0-M viscoelastic parameter $\tau_{1}$ (the long term relaxation time constant).
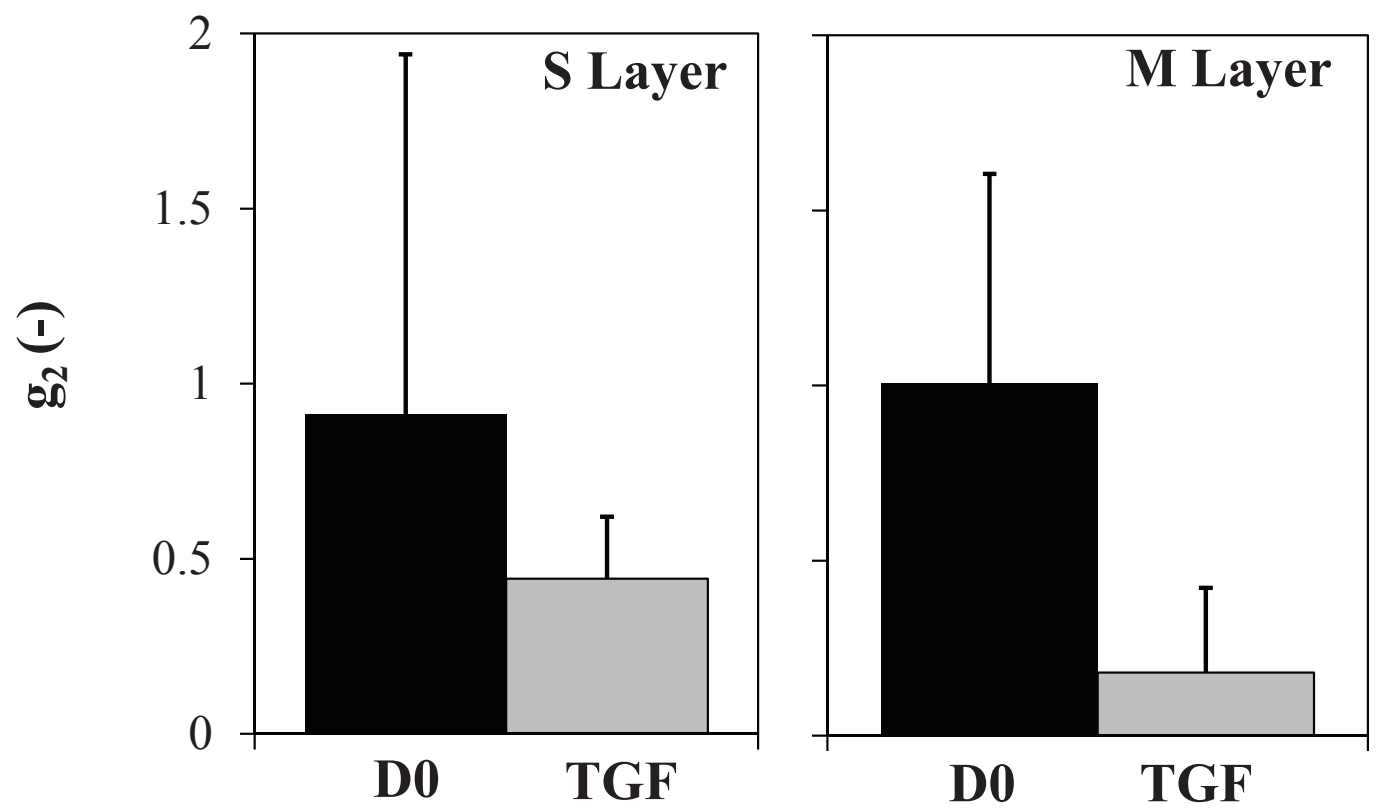

Figure 19: Effect of TGF- $\beta 1$ on D0-S and D0-M viscoelastic parameter $g_{2}$ (the short term relaxation coefficient). 

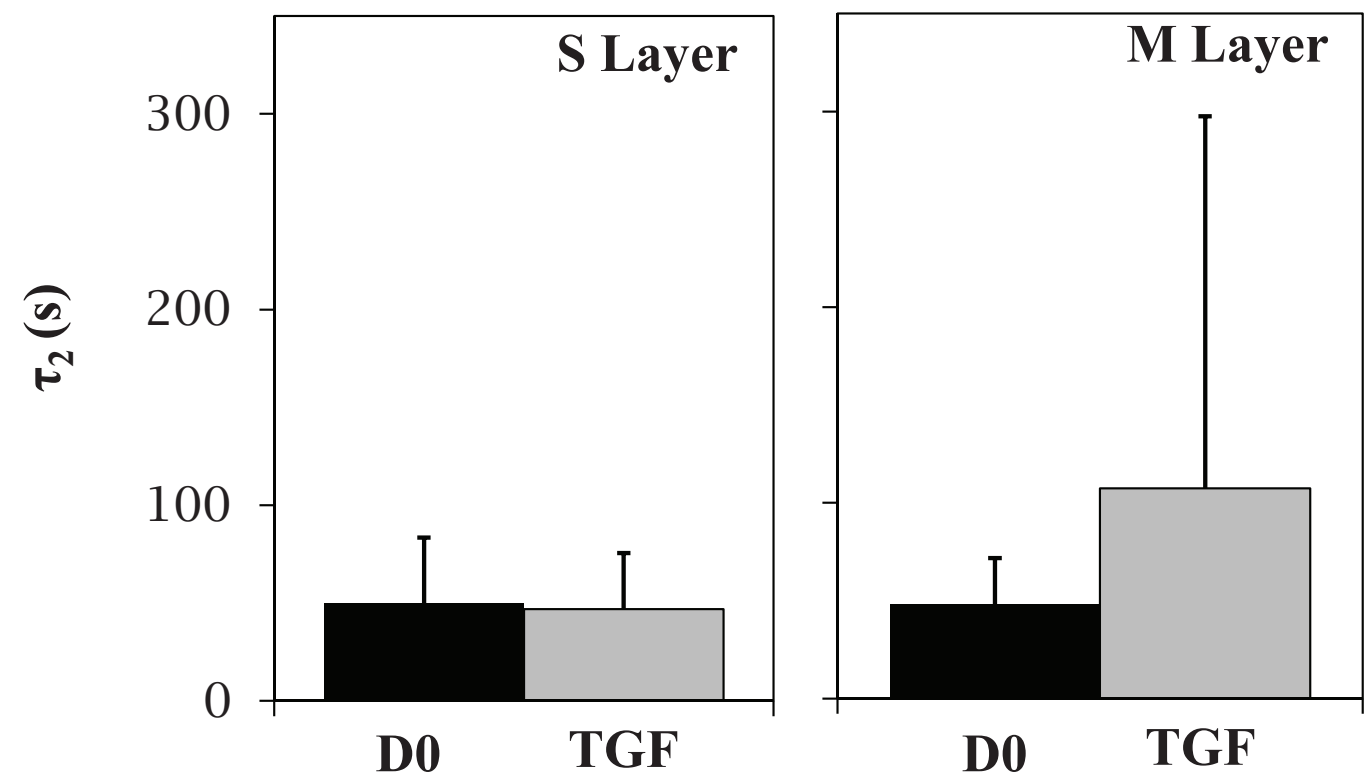

Figure 20: Effect of TGF- $\beta 1$ on D0-S and D0-M viscoelastic parameter $\tau_{2}$ (the short term relaxation time constant). 

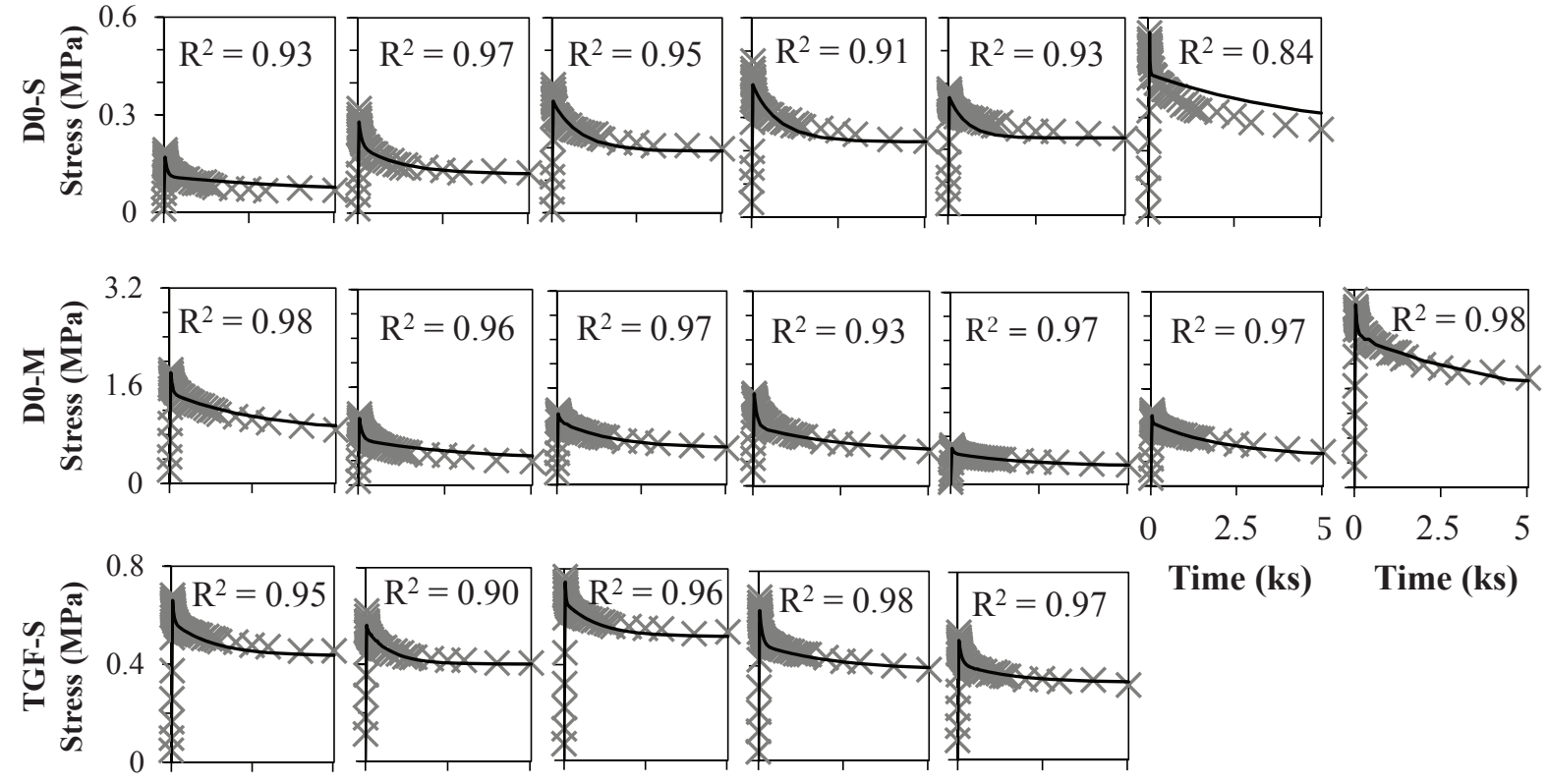

Time (ks) Time (ks)

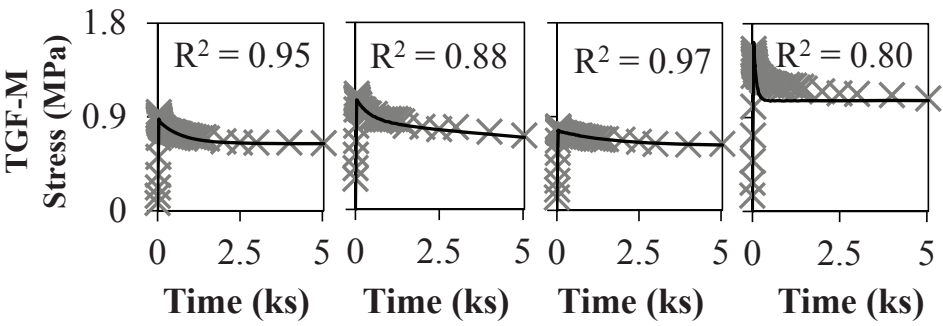

Figure 21: Individual tissue specimen fits. D0 corresponds to untreated explants, TGF to explants treated in transforming growth factor- $\beta 1$. S corresponds to the superficial layer, and $\mathrm{M}$ to the middle layer. Viscoelastic parameters for each tissue source can be found in Appendix D. Note that $\mathrm{y}$-axis scales are different between groups but equal within groups. 


\section{Discussion and Conclusions}

A finite element mixture model was updated to include a viscoelastic collagen constituent with a continuous anisotropic fiber distribution. A quasi-linear viscoelastic model was used to model the intrinsic viscoelasticity of collagen in tension. The updated finite element mixture model proves to be versatile when modeling AC. It captures the GAG-COL interactions reported by Stender [1] and Thomas [69] as well as the intrinsic viscoelasticity of $\mathrm{AC}$ and the anisotropic distribution of $\mathrm{COL}$ fibers. Some study was done on $\mu$ (Appendix D) and although other values achieved similar values of goodness of fit, it was decided to use Stender's findings [1] to maintain continuity. Stender [1] showed that $\mu=0.001$ achieved the best fit given the constraint that $\mu>0$ in order to prevent loss of convergence.

Results from the quasi-linear viscoelastic model elucidate several effects of TGF$\beta 1$. The first is the trend for treatment in TGF- $\beta 1$ to increase fiber modulus (Figure 16). Middle layer specimens showed an increase that was significantly different $(p<0.05)$, while superficial layer had trend in increase $(p=0.087)$. This increase in COL fiber modulus also caused the tissue as a whole to become stiffer. This phenomenon is also present when analyzing the average of each tissue source as one specimen (Table 4).

Another interesting affect of treatment in TGF- $\beta 1$ is the change in viscosity. TGF- $\beta 1$ causes the tissue to not be able to relax as much, as seen by the decrease in $g_{1}$ (Figure 17) where there is a significant difference between D0 and TGF for both $\mathrm{S}$ and M layers $(p<0.005$ and $p<0.001$ repsectively) and a trend in decrease of $g_{2}$ between D0 and TGF for both $\mathrm{S}$ and M layers $-p=0.648$ and $p=0.221$ respectively - (Figure 19). $g_{1}$ and $g_{2}$ are the amplification coefficients, and when they become smaller, the equilibrium stress (as a percentage of maximum stress) becomes larger. This was also the case when looking at the average of all specimens for each tissue source (i.e. $g_{1}$ and $g_{2}$ decrease after treatment in TGF- $\beta 1$ for both $\mathrm{S}$ and M layers - Table 4). This can also be seen when we generalize the relaxation by the use of $R_{\text {exp }}$, the equilibrium stress divided by the peak stress. Results from 
the single average specimens (Table 5) show that treatment causes $R_{\exp }$ to increase for both $\mathrm{S}$ and $\mathrm{M}$ layers meaning that the equilibrium stress is a higher percentage of the peak stress and therefore not relaxing as much as D0 specimens. Appendix D lists the $R_{\exp }$ for each individual specimen. Table 5 also shows a decrease in $\tau_{\text {exp }}$, meaning that the treated tissue tends to also relax faster. This phenomenon is often described as a loss of viscosity as the tissue is behaving less viscously. One note in these results is the high percentage difference between experimental $\tau_{\text {exp }}$ and model results. These differences are so high because the two-term QLV response can't quite model the relaxation profile of these specimens. These results suggest that perhaps a three-term Prony series expansion would be more appropriate as the relaxation function for the QLV. Although the individual specimens don't show a significant difference between any of the relaxation constants (with only $\tau_{1}$ showing a slight trend in decrease between D0 and TGF- $\beta 1-p=0.915$ for the S layer and $p=0.998$ for the M layer - Figure 18), the average specimens show that both $\tau_{1}$ and $\tau_{2}$ become smaller after treatment in TGF- $\beta 1$ (Table 4) which results in a faster relaxation response (i.e. the tissue relaxes to its equilibrium value more quickly).

These results (i.e. a stiffer, less viscous tissue) suggest that treatment in TGF$\beta 1$ is causing a "maturational" effect. Results from Charlebois et al. [80] show an increase in both equilibrium and peak tensile modulus with age as well as a loss in viscosity (young cartilage could relax entirely whereas adult cartilage retained approximately $60 \%$ of the maximum stress). These results confirm that TGF- $\beta 1$ indeed causes cartilage to exhibit a more mature response in tension, seen here primarily through a maturation of collagen fibers.

The mechanisms for this result are somewhat hard to conclude. Williamson et al. [81] shows a decrease in water percentage and increase in collagen content (normalized to initial wet weight) from fetal to adult cartilage which are similar to trends for D0 to D12-TGF- $\beta 1$ [2]. However, Williamson et al. [81] also shows decrease in glycosaminoglycan content normalized to initial wet weight $\left(\mathrm{GAG} / \mathrm{WW}_{\mathrm{i}}\right)$ and an increase in pyridinoline cross links to collagen (PYR/COL) from fetal to adult cartilage whereas Williams et al. [2] shows that GAG/WW tends to increase with 
application of TGF- $\beta 1$ and there is no noticeable effect on PYR/COL. Thomas [69] hypothesized a maturational effect brought on by GAG depletion and concluded that during maturation their exists GAG-COL interactions allowing for a highly compliant solid matrix to provide for rapid volumetric expansion and may play a role in lower relaxation ratio and enhanced viscous properties.

As part of the experimental procedure, there is an initial tare strain recorded before the specimen is stretched. It was believed that this tare strain was not actually stretching the specimen, but was accounted for by specimen sag and jawslippage. Tare strain was typically on the same order of magnitude as experimental strain, and thus when included caused drastically different results. For these reasons, it was decided to remove tare strain from the simulation but still continue to include the recorded tare stress - since there was no practical way to tell how much of this stress was actually being seen by the specimen. This allowed the model to calculate somewhat better fits. The results of these pilot simulations can be seen in Appendix D.

One of the limitations of this work is that qPLM data was not available for the specific tissue sources modeled. This assumes that not only can the COL fiber distribution be generalized across untreated tissue sources, but that treatment in TGF- $\beta 1$ does not affect the distribution either. It is unlikely that if treatment in TGF- $\beta 1$ causes a more anisotropic distribution that results would change much (since the distribution used is already so highly anisotropic). If, however, treatment in TGF$\beta 1$ shifted the distribution toward isotropy, results may be affected. Stender et al. performed pilot studies with an isotropic distribution and showed that although predicted COL fiber modulus $\left(E_{f}\right)$ increased, the trends remained the same [74]. Future studies of this work should look into how an isotropic distribution might affect the results.

Some loss of convergence issues were still being encountered at the end of the simulation, particularly with tissue samples exhibiting a high concentration of initial GAG density or more complex relaxation curves. The divergence with high GAG densities could be due to the COL-GAG interaction and the low amount of COL in 
the lateral direction (with the new implementation of an anisotropic distribution). Some of the complex relaxation curves suggest the use of a third term in the Prony series expansion, however this raises uniqueness concerns. Already, individual results are not unique (as the simplex optimization only guarantees local uniqueness). A three-term Prony series would be even harder to guarantee uniqueness, especially using only one relaxation curve. It is suggested that future studies look into trying a more complete set of starting vertices to try to guarantee global convergence of the simplex. In addition, future studies should look at comparing to successive relaxation curves to guarantee uniqueness of each tissue solution.

The lack of significant difference between viscoelastic parameters for the individual specimens is most likely due to the high variability of data and low number of specimens. For this reason, it may be better to use the average of each tissue specimen as a single tissue in order to generalize the effects of TGF- $\beta 1$. This allows the variability to have less significance. A beneficial study might look into how many specimens are needed until the results of both the individual specimens and the average specimen coincide; future studies could also look into modeling more specimens from each tissue source.

Studies show that cartilage becomes stiffer and less viscous with age [80] and this study shows that growth in TGF- $\beta 1$ has a similar effect. In reference to the goals of this study (Section 1.3), the COL constituent of previous AC mixture models was successfully updated to model the intrinsic viscoelasticity of COL fibers and shown to adequately predict the stress relaxation of $\mathrm{AC}$ in tension. Future studies could look into expanding this model. With the addition of permeability, this model could easily model both unconfined and confined compression to further study the role of intrinsic viscoelasticity of collagen fibers as well as flow-dependent viscoelasticity and the overall nature of articular cartilage. Such a biphasic model would more accurately describe the physical characteristics of articular cartilage. 


\section{REFERENCES}

[1] Stender, M.E. "Predicting articular cartilage constituent material properties following in vitro growth using a proteoglycan-collagen mixture model." M.S.M.E. thesis, California Polytechnic State University, San Luis Obispo, CA, 2011.

[2] Williams, G.M., Dills, K.J., Flores, C.R., Stender, M.E., Stewart, K.M., Nelson, L.M., Chen, A.C., Masuda, K., Hazelwood, S.J., Klisch, S.M., Sah, R.L. (2010). Differential regulation of immature articular cartilage compressive moduli and Poisson's ratios by in vitro stimulation with IGF-1 and TGF- $\beta 1$. Journal of Biomechanics, 43:2501-2507.

[3] Stender, M.E., Balcom, N., Berg-Johansen, B., Dills, K.J., Dyk, D., Sah, R.L., Klisch, S.M., Hazelwood, S.J. Differential regulation of articular cartilage tensile properties by IGF-1 and TGF- $\beta 1$ during in vitro growth. Submitted toInternational Conference on the Mechanics of Biomaterials and Tissues, 2011.

[4] Kumar, P., Oka, M., Toguchida, J., Kobayashi, M., Uchida, E., Nakamura, T., Tanaka, K. (2001). Role of uppermost superficial surface layer of articular cartilage in the lubrication mechanicsm of joints. Journal of Anatomy. 199:241250.

[5] Herzog, W., Federico, S.(2006). Considerations on Joint and Articular Cartilage Mechanics. Biomechanics and Modeling in Mechanobiology, 5(2-3):64-81.

[6] Cheng, Y., Hootman, J., Murphy, L., Langmaid, G., and Helmick, C. (2010). Prevalence of Doctor-Diagnosed Arthritis and Arthritis-Attributable Activity Limitation. CDC: Morbidity and Mortality Weekly Report, 59(39):1261-1265.

[7] Buckwalter, J. A., Mankin, H. J. (1998). Articular cartilage repair and transplantation. Arthritis \& Rheumatism, 41(8):1331-1342. 
[8] Hunziker, E. B. (2002). Articular cartilage repair: basic science ad clinical progress. A review of the current status and prospects. Osteoarthritis and Cartilage,10:432-463.

[9] "Total Knee Replacement Surgery." Internet: http://my.clevelandclinic. org/services/knee_replacement/hic_total_knee_replacement_surgery . aspx, 2010 [July 20, 2010].

[10] Ficklin, T., Thomas, G., Barthel, J.C., Thonar, E.J., Masuda, K., Asanbaeva, A., Chen, A.C., Sah, R.L., Davol, A., Klisch, S.M. (2007). Articular cartilage mechanical and biochemical property relations before and after in vitro growth. Journal of Biomechanics, 40:3607-3614.

[11] Soltz, M.A., Ateshian, G.A. (2000). A cone wise linear elasticity model for the analysis of tension-compression nonlinearity of articular cartilage. Journal of Biomechanical Engineering, 122(6):576-586.

[12] Klein, T.J., Chaudry, M., Bae, W.C., Sah, R.L.(2007). Depth-dependent biomechanics and biochemical properties of fetal, newborn, and tissue- engineered articular cartilage. Journal of Biomechanics, 40(1):182-190.

[13] Schinagi, R.M., Gurskis, D., Chen, A.C., Sah, R.L. (1997). Depth-dependent confined compression modulus of full-thickness bovine articular cartilage. Journal of Orthopaedic Research, 15(4):499-506.

[14] Armstrong, C.G., Mow, V.C. (1997). Variations in the intrinsic mechanical properties of human articular cartilage with age, degeneration, and water content. Journal of Bone and Joint Surgery, American Volume, 64(1):88-94.

[15] Han, L., Frank, E.H., Greene, J.J., Lee, H-Y., Hung, HH.K., Grodzinsky, A.J., Ortiz, C. (2011). Time-dependent nanomechanics of cartilage. Biophysical Journal, 100:1846-1854.

[16] Hayes, W.C., Mockros, L.F. (1971). Viscoelastic properties of human articular cartilage. Journal of Applied Physiology, 31(4):562-568. 
[17] Klisch, S.M. Cal Poly Cartilage Biomechanics Group, Internet: http://www. calpoly.edu/ sklisch/biogroup/index.htm, 2008 [July 21, 2011].

[18] Sah, R.L., Chen, A.C., Chen, S.S., Li. K.W., DiMicco, M.A., Kurtis, M.S., Lottman, L.M., and Sandy, J.D. (2001). "Articular Cartilage Repair." Arthritis and Allied Conditions. A Textbook of Rheumatology. W.J. Koopman, ed., Lippincott, Williams \& Wilkins, Philadelphia, pp. 2264-2278.

[19] Smith, G.D., Knutsen, G., and Richardson, J.B. (2005). A clinical review of cartilage repair techniques. Journal of Bone and Joint Surgery British. 87B(4): 445-449.

[20] Asanbaeva, A., Masuda, K., Thonar, E.J., Klisch, S.M., Sah, R.L. (2008). Regulation of immature cartilage growth by IGF-1, TGF- $\beta 1$, BMP-7, and PDGFAB: Role of metabolic balance between fixed charge and collagen network. Biomechanics and Modeling in Mechanobiology. 7(4): 263-276.

[21] Mak, A.F. (1986). The apparent viscoelastic behavior of articular cartilage the contributions from the intrinsic matrix viscoelasticity and interstitial fluid flows. Journal of Biomechanical Engineering, 108(2):123-130.

[22] Huang, C-Y., Mow, V.C., Ateshian, G.A (2001). The role of flow-independent viscoelasticity in the biphasic tensile and compressive responses of articular cartilage. Journal of Biomechanical Engineering, 123(5):410-417.

[23] Kam, K.K. "Poroelastic finite element analysis of a heterogeneous articular cartilage explant under dynamic compression in Abaqus." M.S.M.E. thesis, California Polytechnic State University, San Luis Obispo, CA, 2011.

[24] Li, L.P., Herzog, W. (2004). The role of viscoelasticity of collagen fibers in articular cartilage: Theory and numerical formulation. Biorheology. 41(3-4): 181-194.

[25] Klisch, S.M. (2007). A bimodular polyconvex anisotropic strain energy function for articular cartilage. Journal of Biomechanical Engineering. 129:250-258. 
[26] Bartel, D.L., Davy, D.T., Keaveny, T.M. "Tissue Mechanics II: Soft Tissue," in Orthopeadic Biomechanics: Mechanics and Design in Musculoskeletal Systems, $1^{\text {st }}$ ed. Upper Saddle River, NJ: Pearson Prentice Hall, 2006, ch. 4, pp. 131-163.

[27] Mow, V.C., Kuei, S.C., Lai, W.M., Armstrong, C.G. (1980). Biphasic creep and stress relaxation of articular cartilage in compression: theory and experiments. Journal of Biomechanical Engineering. 102(1): 73-84.

[28] Kwan, M.K., Lai, M.W., Mow, V.C. (1990). A finite deformation theory for cartilage and other soft hydrated connective tissues - I. Equilibrium results. Journal of Biomechanical Engineering. 23: 124-155.

[29] Truesdell, C., Toupin, R.A. "The Classical Field Theories." Handbuch der Physik, S. Flügge, ed., Springer-Verlag, Berlin. 1960.

[30] Green, A.E., Naghdi, P.M. (1968). A note on mixtures. International Journal of Engineering Science. 6: 631-635.

[31] Green, A.E., Naghdi, P.M. (1969). On basic equations for mixtures. Quarterly Journal of Mechanics \& Applied Mathematics. 22: 427-438.

[32] Bowen, R.M. (1980). Incompressible porous media models by use of the theory of mixtures. International Journal of Engineering Science. 18: 1129-1148.

[33] Müller, I. (1968). A thermodynamic theory of mixtures of fluids. Archive for Rational Mechanics and Analysis. 28: 1-39.

[34] Craine, R.E, Green, A.E., Naghdi, P.M. (1970). A mixture of viscous elastic materials with different constituent temperatures. Quarterly Journal of Mechanics E6 Applied Mathematics. 23(2): 171-184.

[35] Mills, N. (1966). Incompressible mixture of newtonian fluids. International Journal of Engineering Science. 4: 97-112. 
[36] Holems, M.H., Mow, V.C. (1990). The nonlinear characteristics of soft gels and hydrated connective tissues in ultrafiltration. Journal of Biomechanics. 23(11): $1145-1156$.

[37] Ateshian, G.A., Warden, W.H., Kim, J.J., Grelsamer, R.P., Mow, V.C. (1997). Finite deformation biphasic material properties of bovine articular cartilage from confined compression experiments. Journal of Biomechanics. 30(11-12): $1157-1164$.

[38] Klisch, S.M., Lotz, J.C. (2000). A special theory of biphasic mixtures and experimental results for human annulus fibrosus tested in confined compression. Journal of Biomechanical Engineering. 122: 180-188.

[39] Hayes, W.C., Bodine, A.J. (1978). Flow-independent viscoelastic properties of articular cartilage matrix. Journal of Biomechanics. 11: 407-419.

[40] Li, L.P., Soulhat, J., Buschmann, M.D., Shirazi-Adl, A. (1999). Nonlinear analysis of cartilage in unconfined ramp compression using a fiber reinforced poroelastic model. Clinical Biomechanics. 14: 673-682.

[41] Li, L.P., Herzog, W., Korhonen, R.K., Jurvelin, J.S. (2005). The role of viscoelasticity of collagen fibers in articular cartilage: axial tension versus compression. Medical Engineering and Physics. 51-57.

[42] Fung, Y.C. (1973). Biorheology of soft tissues. Biorheology. 10: 139-155.

[43] Suh, J.K., Bai, S. (1998). Finite element formulation of biphasic poroviscoelastic model for articular cartilage. Journal of Biomechanical Engineering. 120: 195201.

[44] Wilson, W., van Donkelaar, C.C., van Rietbergen, B., Ito, K., Huiskies, R. (2004). Stresses in the local collagen network of articular cartilage: a poroviscoelastic fiber reinforced finite element study. Journal of Biomechanics. 37(3): $357-366$ 
[45] García, J.J., Cortés, D.H. (2006). A nonlinear biphasic viscohyperelastic fiberreinforced model for articular cartilage: Formulation and comparison with experimental data. Journal of Biomechanics. 37: 357-366.

[46] DiSilvestro, M.R., Zhu, Q., Wong, M., Jurvelin, J.S., Suh, J.F. (2001). Biphasic poroviscoelastic simulation of the unconfined compression of articular cartilage: I - Simultaneous prediction of reaction force and lateral displacement. Journal of Biomechanical Engineering. 123: 191-197.

[47] Trickey, W.R., Lee, G.M., Guilak, F. (2005). Viscoelastic properties of chondorocytes from normal and osteoarthritic human cartilage. Journal of Orthopaedic Research. 18(6): 891-898.

[48] Lei, F., Szeri, A.Z. (2007). Predicting articular cartilage behavior with a nonlinear microstructural model. The Open Mechanics Journal. 1: 11-19.

[49] Park, S., Ateshian, G. (2006). Dynamic response of immature bovine articular cartilage in tension and compression, and nonlinear viscoelastic modeling of the tensile response. Journal of Biomechanical Engineering. 128(4): 623-630.

[50] Pryse, K.M., Nekouzadeh, A., Genin, G.M., Elson, E.L., Zahalak, G.I. (2003). Incremental mechanics of collagen gels: New experiments and a new viscoelastic model. Annals of Biomedical Engineering. 31: 1287-1296.

[51] Vena, P., Gastaldi, D., Contro, R. (2006). A constituent-based model for the nonlinear viscoelastic behavior of ligaments. Journal of Biomechanical Engineering. 128: 449-457.

[52] Nekouzadeh, A., Pryse, K.M., Elson, E.L., Genin, G.M. (2007). A simplified approach to quasi-linear viscoelastic modeling. Journal of Biomechanics. 40: $3070-3078$

[53] Puso, M.A., Weiss, J.A. (1998). Finite element implementation of anisotropic quasi-linear viscoelasticity using a discrete spectrum approximation. Journal of Biomechanical Engineering. 120: 62-70. 
[54] Minns, R.J., Steven, F.S. (1977). The collagen fiber organization in human articular cartilage. Journal of Anatomy. 123(2): 437-457.

[55] Benninghoff, A. (1925). Form und bau der gelenkknorpel in ihren beziehungen zur funktion. Cell and Tissue Research. 2(5): 783-862.

[56] Lei, F., Szeri, A.Z. (2006). The influence of fiber organization on the mechanical behaviour of articular cartilage. Proceedings of the Royal Society. 462: 33013322 .

[57] Ateshian, G.A., Raja, V., Chahine, N.O., Canal, C.E., Hung, C.T. (2009). Modeling the matrix of articular cartilage using a continuous fiber angular distribution predicts many observed phenomena. Journal of Biomechanical Engineering. 131: 061003-1-061003-10.

[58] Shirazi, R., Vena, P., Sah, R.L., Klisch, S.M. (2011). Modeling the collagen fiber network of biological tissues as a nonlinearly elastic material using a continuous volume fraction distribution function. Mathematics and Mechanics of Solids. [Online]. Available: http://mms.sagepub.com/content/early/2011/04/01/ 1081286510387866 . abstract. [July 21, 2011].

[59] Julkunen, P., Iivarinen, J., Brama, P.A., Arokoski, J., Jurvelin, J.S., Helminen H.J. (2010). Maturation of collagen fibril network structure in tibial and femoral cartilage of rabbits. Osteoarthritis and Cartilage. 18(3): 406-415.

[60] Rieppo, J., M.D., Hyttinen, M.M., M.D., Halmesmaki, E., M.D., Ruotsalainen, H., M.Sc., Vasara, A., M.D., Ph.D., Kiviranta, I., M.D., Ph.D., Jurvelin, J.S., Ph.D., Helminen, H.J., M.D., Ph.D. (2009). Changes in spatial collagen content and collagen network architecture in porcine articular cartilage during growth and maturation. Osteoarthritis and Cartilage. 17(4): 448-455.

[61] van Turnhout, M.C., Schipper, H., Engel, B., Buist, W., Kranenbarg, S., van Leeuwen, J.L. (2010). Postnatal development of collagen structure in ovine articular cartilage. BMC Developmental Biology. 10:62. 
[62] Huiskes, R., Chao, E.Y.S. (1982). A survey of finite element analysis in orthopedic biomechanics: The first decade. Journal of Biomechanics. 16(6): 385-409.

[63] Abaqus/CAE version 6.10. Providence, Rhode Island: Dassault Systèmes, 2011.

[64] Wu, J.Z., Herzog, W., Epstein, M. (1997). Evaluation of the finite element software Abaqus for biomechanical modeling of biphasic tissues. Journal of Biomechanics. 31(2): 165-169.

[65] Mase, G.T., Smelser, R.E., Mase, G.E. Continuum Mechanics for Engineers. Third Ed. Boca Raton: CRC Press, 2010.

[66] Timoshenko, S. Theory of Elasticity. Third Ed. McGrawHill, 1970.

[67] Buschmann, M., Grodzinksy, A. (1995). A molecular model of proteoglycanassociated forces in cartilage mechanics. Journal of Biomechanical Engineering. 117: 179-192.

[68] Yamauchi, K. "Prediction of articular cartilage growth and remodeling during dynamic unconfined compression with a finite element model." M.S.M.E. thesis, California Polytechnic State University, San Luis Obispo, CA, 2012.

[69] Thomas, G.C., Asanbaeva, A., Vena, P., Sah, R.L., Klisch, S.M. (2009). A nonlinear constituent based viscoelastic model for articular cartilage and analysis of tissue remodeling due to altered glycosaminoglycan-collagen interactions. Journal of Biomechanical Engineering. 131:101002.

[70] Klisch, S.M., Asanbaeva, A., Oungoulian, S.R., Masuda, K., Thonar, E.J., Davol, A., Sah, R.L. (2008). A cartilage growth mixture model with collagen remodeling: validation protocols. Journal of Biomechanical Engineering. 130: 031006.

[71] Ficklin, T.P., Davol, A., Klisch, S.M. (2009). Simulating the growth of articular cartilage explants in a permeation bioreactor to aid in experimental protocol design. Journal of Biomechanical Engineering. 131:041008. 
[72] Oungoulian, R.S. "A polyconvex strain energy function for proteoglycan and validation of a growth mixture model with collagen remodeling." M.S.M.E. thesis, California Polytechnic State University, San Luis Obispo, 2007.

[73] Atkin, R.J., Craine, R.E. (1976). Continuum theories of mixtures: Basic theory and historical development. Quarterly Journal of Mechanics and Applied Mathematics. 29(2): 209-244.

[74] Stender, M.E., Raub, C., Shirazi, R., Yamauchi, K., Vena, P., Sah, R.L., Hazelwood, S.J., Klisch, S.M. (2012). Effects of TGF- $\beta 1$ and IGF-1 on articular cartilage collagen fiber modulus: FEA predicitions using continuous anisotropic fiber distribution with biochemical content, qPLM, and mechanical test data. to be published.

[75] Basser, J.P., Schneiderman, R., Bank, A.R., Wachtel, E., Maroudas, A. (1998). Mechanical properties of the collagen network in human articular cartilage as measured by osmotic stress technique. Archives of Biochemistry and Biophysics. 351(2): 207-219.

[76] MATLAB version 2011a. Natick, Massachusetts: MathWorks, Inc. 2011.

[77] Rosenbrock, H.H. (1960). An automatic method for finding the greatest or least value of a function. The Computer Journal. 3(3): 175-184.

[78] Fletcher, R., Powell, M.J.D. (1963). A rapidly convergent descent method for minimization. The Computer Journal. 6(2): 163-168.

[79] Vena, P., private communication, Dec. 2011.

[80] Charlebois, M., McKee, M.D., Buschmann, M.D. (2004). Nonlinear tensile properties of bovine articular cartilage and their variation with age and depth. Journal of Biomechanical Engineering. 126: 129-137.

[81] Williamson, A.K., Chen, A.C., Masuda, K., Thonar, E.J., Sah, R.L. (2003). Tensile mechanical properties of bovine articular cartilage variations with 
growth and relationships to collagen network components. Journal of Orthopedic Research. 2: 872-880.

[82] Nelder, J.A., Mead, R. (1965). A simplex method for function minimization. The Computer Journal. 7(4): 308-313.

[83] Koay, E.J., Shieh, A.C., Athanasiou, K.A. (2003). Creep indentation of single cells. Journal of Biomechanical Engineering. 125(3): 334-341.

[84] Balooch, M., Wu-Magidi, I-C., Balazs, A., Lundkvist, A.S., Marshall, S.J., Marshall, G.W., Siekhaus, W.J., Kinney, J.H. (1998). Viscoelastic properties of demineralized human dentin measured in water with atomic force microscope (AFM)-based indentation. Journal of Biomedical Materials Research. 40(4): $539-544$.

[85] Thornton, G.M., Oliynyk, A., Frank, C.B., Shrive, N.G. (2005). Ligament creep cannot be predicted from stress relaxation at low stress: A biomechanical study of the rabbit medial collateral ligament. Journal of Orthopaedic Research. 15(5): $652-656$. 


\section{A Derivations}

\section{A.1 Two-Term Prony Series Expansion of the QLV}

If we start with the fiber stress at the current time represented as

$$
\sigma^{v}(t)=\sigma^{v}(0)+\int_{0}^{t} g(t-\xi) \frac{\mathrm{d} \sigma^{e}}{\mathrm{~d} \xi} \mathrm{d} \xi
$$

where the relaxation function is again

$$
g(t+\Delta t-\xi)=g_{\infty}+g_{1} e^{[\xi-(t+\Delta t)] / \tau_{1}}+g_{2} e^{[\xi-(t+\Delta t)] / \tau_{2}} .
$$

The integral in Equation 29 can be separated into two integrals

$$
\begin{aligned}
\int_{0}^{t+\Delta t} g(t+\Delta t-\xi) \frac{\mathrm{d} \sigma^{e}}{\mathrm{~d} \xi} \mathrm{d} \xi= & \int_{0}^{t} g(t+\Delta t-\xi) \frac{\mathrm{d} \sigma^{e}}{\mathrm{~d} \xi} \mathrm{d} \xi \\
& +\int_{t}^{t+\Delta t} g(t+\Delta t-\xi) \frac{\mathrm{d} \sigma^{e}}{\mathrm{~d} \xi} \mathrm{d} \xi
\end{aligned}
$$

$\frac{\mathrm{d} \sigma^{e}}{\mathrm{~d} \xi}$ can be approximated with its first order Taylor series expansion in the second integral

$$
\int_{t}^{t+\Delta t} g(t+\Delta t-\xi) \frac{\mathrm{d} \sigma^{e}}{\mathrm{~d} \xi} \mathrm{d} \xi=\frac{\sigma^{e}(t+\Delta t)-\sigma^{e}(t)}{\Delta t} \int_{t}^{t+\Delta t} g(t+\Delta t-\xi) \mathrm{d} \xi
$$

which upon plugging in Equation 62, the integral becomes

$$
\begin{aligned}
\int_{t}^{t+\Delta t} g(t+\Delta t-\xi) \mathrm{d} \xi=\int_{t}^{t+\Delta t} g_{\infty} \mathrm{d} \xi+\int_{t}^{t+\Delta t} & g_{1} e^{[\xi-(t+\Delta t)] / \tau_{1}} \mathrm{~d} \xi \\
& +\int_{t}^{t+\Delta t} g_{2} e^{[\xi-(t+\Delta t)] / \tau_{2}} \mathrm{~d} \xi
\end{aligned}
$$

The first integral is easily solved

$$
\int_{t}^{t+\Delta t} g_{\infty} \mathrm{d} \xi=g_{\infty} \Delta t
$$


If we separate the exponent in the second integral

$$
\int_{t}^{t+\Delta t} g_{1} e^{[\xi-(t+\Delta t)] / \tau_{1}} \mathrm{~d} \xi=g_{1} \int_{t}^{t+\Delta t} e^{\xi / \tau_{1}} e^{-(t+\Delta t) / \tau_{1}} \mathrm{~d} \xi
$$

from which we can pull out $e^{-(t+\Delta t) / \tau_{1}}$ since it does not depend on $\xi$. This leaves

$$
g_{1} \int_{t}^{t+\Delta t} e^{\xi / \tau_{1}} e^{-(t+\Delta t) / \tau_{1}} \mathrm{~d} \xi=g_{1} e^{-(t+\Delta t) / \tau_{1}} \int_{t}^{t+\Delta t} e^{\xi / \tau_{1}} \mathrm{~d} \xi
$$

The integral is easily solved

$$
\int_{t}^{t+\Delta t} e^{\xi / \tau_{1}} \mathrm{~d} \xi=\left.\tau_{1} e^{\xi / \tau_{1}}\right|_{t} ^{t+\Delta t}=\tau_{1}\left(e^{t+\Delta t / \tau_{1}}-e^{t / \tau_{1}}\right)
$$

leaving the second integral from Equation 65 as

$$
\int_{t}^{t+\Delta t} g_{1} e^{[\xi-(t+\Delta t)] / \tau_{1}} \mathrm{~d} \xi=g_{1} \tau_{1} e^{-(t+\Delta t) / \tau_{1}}\left(e^{t+\Delta t / \tau_{1}}-e^{t / \tau_{1}}\right)
$$

which upon distributing $e^{-(t+\Delta t) / \tau_{1}}$ is written as

$$
\begin{array}{r}
g_{1} \tau_{1} e^{-(t+\Delta t) / \tau_{1}}\left(e^{t+\Delta t / \tau_{1}}-e^{t / \tau_{1}}\right)=g_{1} \tau_{1}\left(e^{t+\Delta t / \tau_{1}} e^{-(t+\Delta t) / \tau_{1}}\right. \\
\left.-e^{t / \tau_{1}} e^{-(t+\Delta t) / \tau_{1}}\right)
\end{array}
$$

and can finally be reduced to

$$
\int_{t}^{t+\Delta t} g_{1} e^{[\xi-(t+\Delta t)] / \tau_{1}} \mathrm{~d} \xi=g_{1} \tau_{1}\left(1-e^{-\Delta t / \tau_{1}}\right) .
$$

Similarly, the third integral of Equation 65

$$
\int_{t}^{t+\Delta t} g_{2} e^{[\xi-(t+\Delta t)] / \tau_{2}} \mathrm{~d} \xi=g_{2} \tau_{2}\left(1-e^{-\Delta t / \tau_{2}}\right) .
$$

so that the integral of Equation 64 is now 


$$
\begin{aligned}
\int_{t}^{t+\Delta t} g(t+\Delta t-\xi) \mathrm{d} \xi=\frac{\sigma^{e}(t+\Delta t)-\sigma^{e}(t)}{\Delta t}[ & g_{\infty} \Delta t+g_{1} \tau_{1}\left(1-e^{-\Delta t / \tau_{1}}\right) \\
& \left.+g_{2} \tau_{2}\left(1-e^{-\Delta t / \tau_{2}}\right)\right]
\end{aligned}
$$

We now split the first integral of Equation 63 into its respective parts

$$
\begin{aligned}
\int_{0}^{t} g(t+\Delta t-\xi) \frac{\mathrm{d} \sigma^{e}}{\mathrm{~d} \xi} \mathrm{d} \xi=\int_{0}^{t} g_{\infty} \frac{\mathrm{d} \sigma^{e}}{\mathrm{~d} \xi} \mathrm{d} \xi & +\int_{0}^{t} g_{1} e^{\frac{\xi-(t+\Delta t)}{\tau_{1}}} \frac{\mathrm{d} \sigma^{e}}{\mathrm{~d} \xi} \mathrm{d} \xi \\
& +\int_{0}^{t} g_{2} e^{\frac{\xi-(t+\Delta t)}{\tau_{2}}} \frac{\mathrm{d} \sigma^{e}}{\mathrm{~d} \xi} \mathrm{d} \xi
\end{aligned}
$$

The first integral is easily solved as

$$
\int_{0}^{t} g_{\infty} \frac{\mathrm{d} \sigma^{e}}{\mathrm{~d} \xi} \mathrm{d} \xi=g_{\infty}\left(\sigma^{e}(t)-\sigma^{e}(0)\right)
$$

If we establish the following parameter

$$
c_{1}(t)=\int_{0}^{t} g_{1} e^{\frac{\xi-t}{\tau_{1}}} \frac{\mathrm{d} \sigma^{e}}{\mathrm{~d} \xi} \mathrm{d} \xi
$$

then we can rewrite the second integral from Equation 75 as

$$
\int_{0}^{t} g_{1} e^{\frac{\xi-(t+\Delta t)}{\tau_{1}}} \frac{\mathrm{d} \sigma^{e}}{\mathrm{~d} \xi} \mathrm{d} \xi=e^{\frac{-\Delta t}{\tau_{1}}} c_{1}(t)
$$

Next, let us split $c_{1}(t)$ into two separate integrals

$$
c_{1}(t)=\int_{0}^{t} g_{1} e^{\frac{\xi-t}{\tau_{1}}} \frac{\mathrm{d} \sigma^{e}}{\mathrm{~d} \xi} \mathrm{d} \xi=\int_{0}^{t-\Delta t} g_{1} e^{\frac{\xi-t}{\tau_{1}}} \frac{\mathrm{d} \sigma^{e}}{\mathrm{~d} \xi} \mathrm{d} \xi+\int_{t-\Delta t}^{t} g_{1} e^{\frac{\xi-t}{\tau_{1}}} \frac{\mathrm{d} \sigma^{e}}{\mathrm{~d} \xi} \mathrm{d} \xi
$$

If we add and subtract a $\Delta t$ from the exponential (effectively adding zero and thus having no effect), the first integral of this equation can be rewritten as

$$
\int_{0}^{t-\Delta t} g_{1} e^{\frac{\xi-t}{\tau_{1}}} \frac{\mathrm{d} \sigma^{e}}{\mathrm{~d} \xi} \mathrm{d} \xi=e^{\frac{-\Delta t}{\tau_{1}}} \int_{0}^{t-\Delta t} g_{1} e^{\frac{\xi-t+\Delta t}{\tau_{1}}} \frac{\mathrm{d} \sigma^{e}}{\mathrm{~d} \xi} \mathrm{d} \xi
$$

We can then rewrite this integral as 


$$
\int_{0}^{t-\Delta t} g_{1} e^{\frac{\xi-t+\Delta t}{\tau_{1}}} \frac{\mathrm{d} \sigma^{e}}{\mathrm{~d} \xi} \mathrm{d} \xi=\int_{0}^{t-\Delta t} g_{1} e^{\frac{\xi-(t-\Delta t)}{\tau_{1}}} \frac{\mathrm{d} \sigma^{e}}{\mathrm{~d} \xi} \mathrm{d} \xi
$$

which is equivalent to $c_{1}(t-\Delta t)$. Therefore

$$
\int_{0}^{t-\Delta t} g_{1} e^{\frac{\xi-t}{\tau_{1}}} \frac{\mathrm{d} \sigma^{e}}{\mathrm{~d} \xi} \mathrm{d} \xi=e^{\frac{-\Delta t}{\tau_{1}}} c_{1}(t-\Delta t)
$$

We can again approximate $\frac{\mathrm{d} \sigma}{\mathrm{d} \xi}$ as its Taylor series expansion

$$
\int_{t-\Delta t}^{t} g_{1} e^{\frac{\xi-t}{\tau_{1}}} \frac{\mathrm{d} \sigma^{e}}{\mathrm{~d} \xi} \mathrm{d} \xi=\frac{\sigma^{e}(t)-\sigma^{e}(t-\Delta t)}{\Delta t} \int_{t-\Delta t}^{t} g_{1} e^{\frac{\xi-t}{\tau_{1}}} \mathrm{~d} \xi
$$

and then

$$
\int_{t-\Delta t}^{t} g_{1} e^{\frac{\xi-t}{\tau_{1}}} \mathrm{~d} \xi=g_{1} e^{\frac{-t}{\tau_{1}}} \int_{t-\Delta t}^{t} e^{\frac{\xi}{\tau_{1}}} \mathrm{~d} \xi
$$

which can be solved as

$$
\int_{t-\Delta t}^{t} e^{\frac{\xi}{\tau_{1}}} \mathrm{~d} \xi=\tau_{1}\left(e^{\frac{t}{\tau_{1}}}-e^{\frac{t-\Delta t}{\tau_{1}}}\right)
$$

Finally

$$
\int_{t-\Delta t}^{t} g_{1} e^{\frac{\xi-t}{\tau_{1}}} \frac{\mathrm{d} \sigma^{e}}{\mathrm{~d} \xi} \mathrm{d} \xi=\frac{\sigma^{e}(t)-\sigma^{e}(t-\Delta t)}{\Delta t}\left[g_{1} \tau_{1}\left(1-e^{\frac{-\Delta t}{\tau_{1}}}\right)\right]
$$

and

$$
c_{1}(t)=e^{\frac{-\Delta t}{\tau_{1}}} c_{1}(t-\Delta t)+\frac{\sigma^{e}(t)-\sigma^{e}(t-\Delta t)}{\Delta t}\left[g_{1} \tau_{1}\left(1-e^{\frac{-\Delta t}{\tau_{1}}}\right)\right] .
$$

Similarly

$$
\int_{0}^{t} g_{2} e^{\frac{\xi-(t+\Delta t)}{\tau_{2}}} \frac{\mathrm{d} \sigma^{e}}{\mathrm{~d} \xi} \mathrm{d} \xi=e^{\frac{-\Delta t}{\tau_{2}}} c_{2}(t)
$$

where $c_{2}(t)$ is defined as

$$
c_{2}(t)=e^{\frac{-\Delta t}{\tau_{2}}} c_{2}(t-\Delta t)+\frac{\sigma^{e}(t)-\sigma^{e}(t-\Delta t)}{\Delta t}\left[g_{2} \tau_{2}\left(1-e^{\frac{-\Delta t}{\tau_{2}}}\right)\right] .
$$


All of this leads to

$$
\int_{0}^{t} g(t+\Delta t-\xi) \frac{\mathrm{d} \sigma^{e}}{\mathrm{~d} \xi} \mathrm{d} \xi=g_{\infty}\left(\sigma^{e}(t)-\sigma^{e}(0)\right)+e^{\frac{-\Delta t}{\tau_{1}}} c_{1}(t-\Delta t)+e^{\frac{-\Delta t}{\tau_{2}}} c_{2}(t)
$$

Combining Equation 74 and 90 we are left with the viscoelastic fiber stress at the current time as

$$
\begin{array}{r}
\sigma^{v}(t+\Delta t)=\sigma_{0}^{e}+g_{\infty}\left(\sigma^{e}(t)-\sigma^{e}(0)\right)+e^{\frac{-\Delta t}{\tau_{1}}} c_{1}(t)+e^{\frac{-\Delta t}{\tau_{2}}} c_{2}(t) \\
+\frac{\sigma^{e}(t+\Delta t)-\sigma^{e}(t)}{\Delta t}\left[\begin{array}{r}
{\left[g_{\infty} \Delta t+g_{1} \tau_{1}\left(1-e^{-\Delta t / \tau_{1}}\right)\right.} \\
\left.+g_{2} \tau_{2}\left(1-e^{-\Delta t / \tau_{2}}\right)\right]
\end{array}\right.
\end{array}
$$




\section{B Convergence Study}

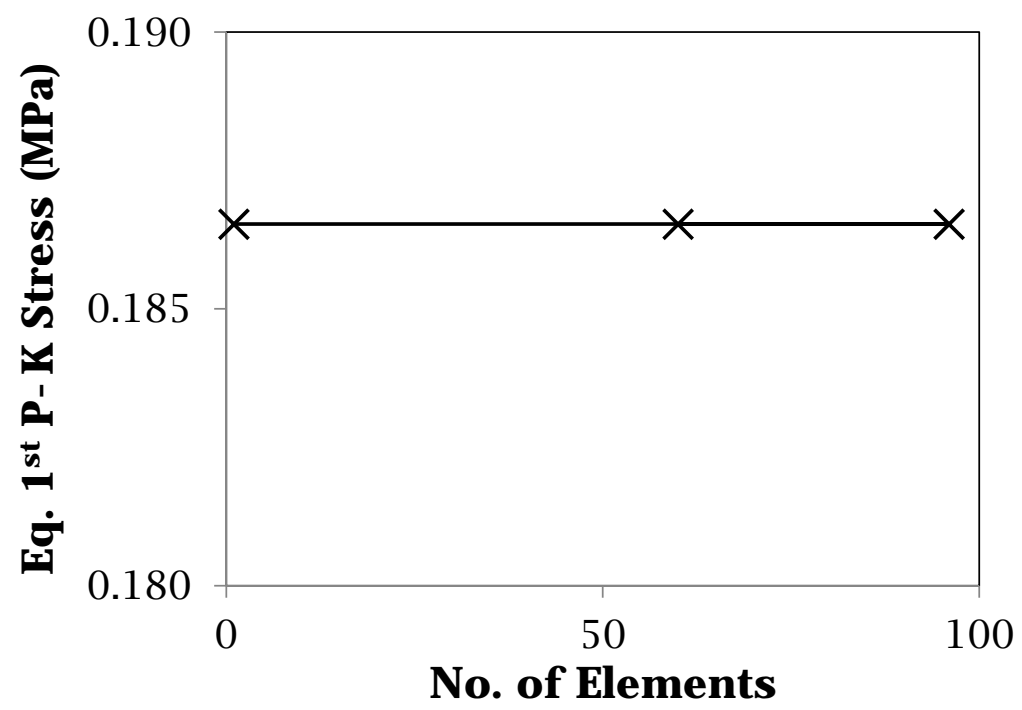

Figure 22: Mesh convergence study. Model is D0-S QLV run with 1, 60, and 96 elements.

The equilibrium stress of all three models was exactly the same (0.1865 MPa). 


\section{Permeability Study}

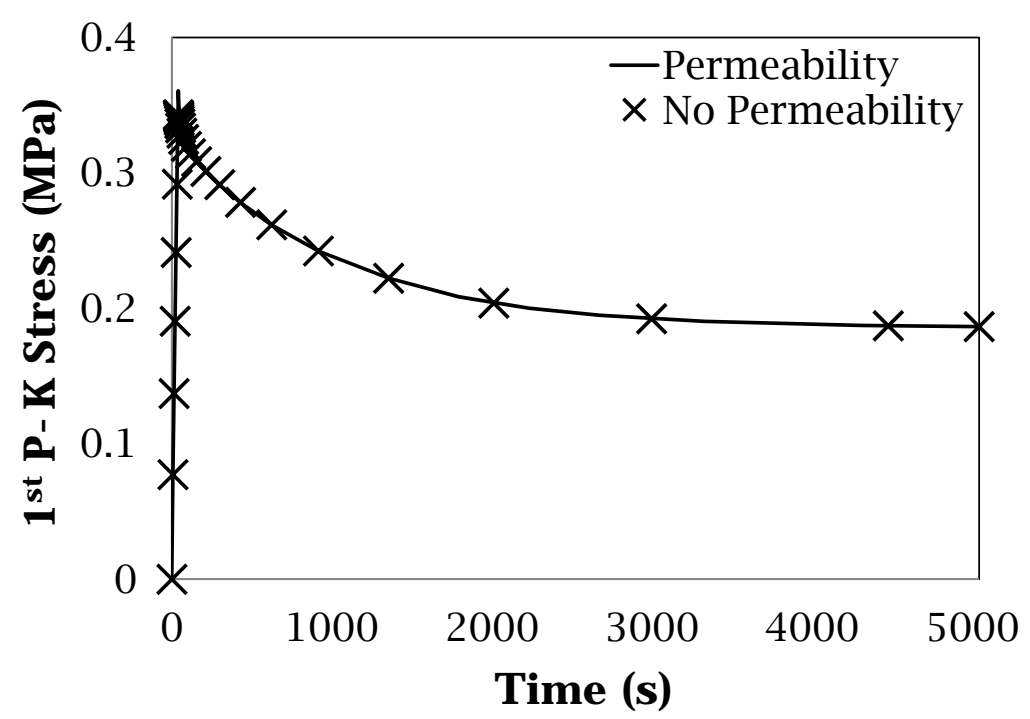

Figure 23: Comparison of D0-S QLV model when including permeability and when not including permeability in tension stress relaxation modeling. Since the exclusion of permeability did not affect results much (the only noticeable difference being a $4.9 \%$ drop in maximum stress), and allows for easier convergence and shorter simulation times it was removed for subsequent simulations.

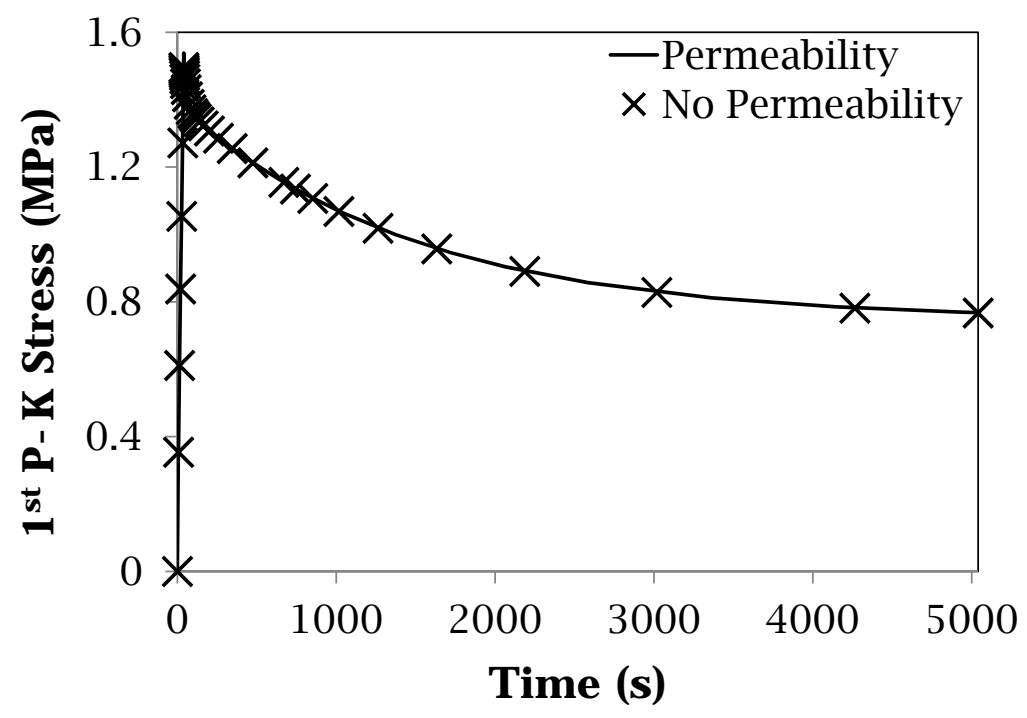

Figure 24: Comparison of D0-M QLV model when including permeability and when not including permeability in tension stress relaxation modeling. Since the exclusion of permeability did not affect results much (the only noticeable difference being a $2.8 \%$ drop in maximum stress), and allows for easier convergence and shorter simulation times it was removed for subsequent simulations. 


\section{Tables and Figures}

Table 8: Specimen specific biochemical data. GAG and COL constituents are normalized to $W W_{f}$. $\phi_{f}$ is calculated using the true density of COL $\left(1.436 \mathrm{~g} / \mathrm{cm}^{3}[75]\right)$. Values from [3]. Tissue samples correspond to results in Figure 21 in order from left to right. In the naming scheme below, D00 corresponds to untreated specimens, and C12 treated, S or M correspond to superficial or middle layer respectively.

\begin{tabular}{|c|c|c|c|}
\hline Tissue & $\begin{array}{c}\text { GAG } \\
\left(\% W W_{f}\right) \\
\end{array}$ & $\begin{array}{c}\mathrm{COL} \\
\left(\% W W_{f}\right) \\
\end{array}$ & $\begin{array}{c}\phi_{f}^{t o t} \\
(\% \text { total tissue volume })\end{array}$ \\
\hline 8D0014ST & 2.631 & 7.568 & 5.17 \\
\hline 7D0014ST & 1.717 & 5.893 & 4.03 \\
\hline 7D0011ST & 4.010 & 6.448 & 4.41 \\
\hline 6D0011ST & 1.953 & 6.578 & 4.50 \\
\hline 5D0016ST & 4.510 & 7.740 & 5.29 \\
\hline 5D0011ST & 3.860 & 3.093 & 5.53 \\
\hline 8D0014MT & 4.863 & 9.447 & 6.46 \\
\hline 8D0011MT & 5.144 & 6.856 & 4.69 \\
\hline 7D0014MT & 4.676 & 7.643 & 5.22 \\
\hline 7D0011MT & 4.956 & 8.314 & 5.68 \\
\hline 6D0016MT & 3.11 & 8.693 & 5.94 \\
\hline 6D0011MT & 5.479 & 8.094 & 5.53 \\
\hline 5D011MT & 5.879 & 10.50 & 7.18 \\
\hline $8 \mathrm{C} 1212 \mathrm{ST}$ & 4.521 & 8.557 & 5.85 \\
\hline 10C1211ST & 2.725 & 1.843 & 1.26 \\
\hline 11C1212ST & 5.449 & 5.447 & 3.72 \\
\hline 13C1213ST & 5.446 & 4.392 & 3.00 \\
\hline 13C1214ST & 3.892 & 3.370 & 2.31 \\
\hline 8C1212MT & 3.752 & 7.532 & 5.15 \\
\hline $9 \mathrm{C} 1212 \mathrm{MT}$ & 5.032 & 1.892 & 1.29 \\
\hline $10 \mathrm{C} 1211 \mathrm{MT}$ & 4.577 & 3.443 & 2.35 \\
\hline $11 \mathrm{C} 1212 \mathrm{MT}$ & 4.712 & 4.799 & 3.28 \\
\hline
\end{tabular}


Table 9: Specimen specific simplex optimization results. Tissue samples correspond to results in Figure 21 in order from left to right. $R^{2}$ is the goodness of fit to the specimen specific experimental data.

\begin{tabular}{|c|c|c|c|c|c|c|}
\hline Tissue & $\begin{array}{c}E_{f} \\
(\mathrm{MPa})\end{array}$ & $\begin{array}{c}g_{1} \\
(-)\end{array}$ & $\begin{array}{c}\tau_{1} \\
(\mathrm{~s})\end{array}$ & $\begin{array}{c}g_{2} \\
(-)\end{array}$ & $\begin{array}{c}\tau_{2} \\
(\mathrm{~s})\end{array}$ & $(-)$ \\
\hline 8D0014ST & 91 & 1.05 & 4358 & 1.91 & 63 & 0.93 \\
\hline 7D0014ST & 345 & 1.04 & 1128 & 1.21 & 73 & 0.97 \\
\hline 7D0011ST & 339 & 1.01 & 762 & 0.04 & 62 & 0.95 \\
\hline 6D0011ST & 645 & 1.05 & 719 & 0.03 & 84 & 0.91 \\
\hline 5D0016ST & 343 & 0.66 & 486 & 0.002 & 1.62 & 0.93 \\
\hline 5D0011ST & 390 & 0.91 & 4353 & 2.28 & 14 & 0.84 \\
\hline 8D0014MT & 1291 & 1.05 & 2478 & 0.56 & 21 & 0.98 \\
\hline 8D0011MT & 720 & 1.03 & 2824 & 1.59 & 77 & 0.96 \\
\hline 7D0014MT & 1072 & 1.05 & 1443 & 0.39 & 53 & 0.97 \\
\hline 7D0011MT & 842 & 1.08 & 2358 & 2.02 & 73 & 0.93 \\
\hline 6D0016MT & 499 & 1.05 & 2012 & 0.60 & 53 & 0.97 \\
\hline 6D0011MT & 706 & 1.56 & 1967 & 1.07 & 15 & 0.97 \\
\hline 5D011MT & 1956 & 1.06 & 3616 & 0.81 & 44 & 0.98 \\
\hline 8C1212ST & 647 & 0.40 & 1258 & 0.43 & 44 & 0.95 \\
\hline 10C1211ST & 3806 & 0.50 & 708 & 0.15 & 16 & 0.90 \\
\hline 11C1212ST & 1141 & 0.34 & 1015 & 0.48 & 22 & 0.96 \\
\hline 13C1213ST & 987 & 0.34 & 2213 & 0.62 & 75 & 0.98 \\
\hline 13C1214ST & 1273 & 0.31 & 1493 & 0.53 & 76 & 0.97 \\
\hline 8C1212MT & 1359 & 0.47 & 660 & 0.13 & 13 & 0.95 \\
\hline 9C1212MT & 3536 & 0.83 & 6430 & 0.54 & 392 & 0.88 \\
\hline 10C1211MT & 2466 & 0.30 & 1715 & 0.03 & 22 & 0.97 \\
\hline 11C1212MT & 3461 & 0.98 & 64 & 0.02 & 1.6 & 0.8 \\
\hline
\end{tabular}


Table 10: Specimen specific generalized relaxation results. $R$ is calculated as the equilibrium stress over the peak stress. $\tau$ is the amount of time for the specimen to relax $63.2 \%$ of its total relaxation. Percent differences for each are the difference from the specimen specific experimental values.

\begin{tabular}{|c|c|c|c|c|c|c|}
\hline Tissue & $\begin{array}{c}R_{\text {exp }} \\
(-)\end{array}$ & $\begin{array}{c}R_{f i t} \\
(-)\end{array}$ & $\begin{array}{c}\% \text { Diff. } \\
(\%)\end{array}$ & $\begin{array}{c}\tau_{\text {exp }} \\
(\mathrm{s})\end{array}$ & $\begin{array}{c}\tau_{f i t} \\
(\mathrm{~s})\end{array}$ & $\begin{array}{c}\% \text { Diff. } \\
(\%)\end{array}$ \\
\hline 8D0014ST & 0.355 & 0.457 & 28.8 & 352 & 255 & 27.6 \\
\hline 7D0014ST & 0.386 & 0.434 & 12.4 & 313 & 518 & 65.7 \\
\hline 7D0011ST & 0.500 & 0.553 & 10.7 & 334 & 778 & 132.9 \\
\hline 6D0011ST & 0.487 & 0.570 & 17.1 & 363 & 803 & 121.3 \\
\hline 5D0016ST & 0.608 & 0.657 & 8.2 & 850 & 554 & 34.8 \\
\hline 5D0011ST & 0.477 & 0.564 & 18.1 & 624 & 853 & 36.6 \\
\hline 8D0014MT & 0.472 & 0.523 & 10.7 & 1080 & 1165 & 7.9 \\
\hline 8D0011MT & 0.331 & 0.437 & 32.1 & 550 & 481 & 12.6 \\
\hline 7D0014MT & 0.490 & 0.534 & 8.8 & 954 & 1185 & 24.3 \\
\hline 7D0011MT & 0.373 & 0.400 & 7.2 & 735 & 367 & 50.0 \\
\hline 6D0016MT & 0.494 & 0.535 & 8.5 & 850 & 1283 & 50.9 \\
\hline 6D0011MT & 0.451 & 0.461 & 2.1 & 1044 & 1416 & 45.1 \\
\hline 5D011MT & 0.583 & 0.583 & 0.0 & 938 & 1343 & 43.2 \\
\hline 8C1212ST & 0.667 & 0.662 & 0.8 & 383 & 657 & 71.3 \\
\hline 10C1211ST & 0.654 & 0.715 & 9.4 & 314 & 696 & 121.6 \\
\hline 11C1212ST & 0.700 & 0.695 & 0.6 & 244 & 571 & 134.2 \\
\hline 13C1213ST & 0.557 & 0.619 & 11.1 & 298 & 199 & 33.2 \\
\hline 13C1214ST & 0.586 & 0.653 & 11.5 & 320 & 353 & 10.6 \\
\hline 8C1212MT & 0.684 & 0.731 & 6.9 & 469 & 0.676 & 44.1 \\
\hline 9C1212MT & 0.639 & 0.658 & 3.0 & 1212 & 1222 & 0.8 \\
\hline 10C1211MT & 0.792 & 0.817 & 3.1 & 944 & 1529 & 61.9 \\
\hline 11C1212MT & 0.694 & 0.656 & 5.5 & 324 & 113 & 65.0 \\
\hline
\end{tabular}




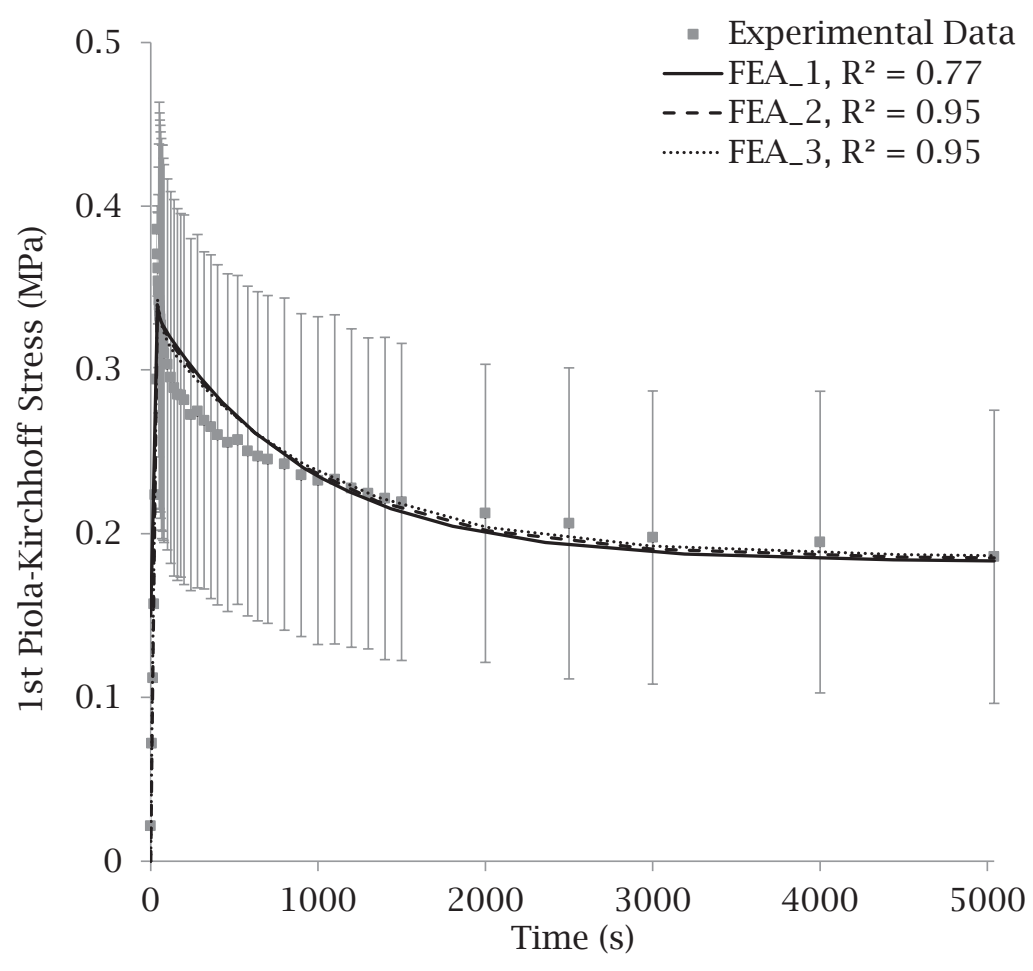

Figure 25: Comparison of QLV models curve fit to stress relaxation data from D0 superficial layer bovine articular cartilage under tensile load. Test protocols are described in 32. Experimental data is the average of six untreated specimens harvested from the patellarfemoral groove; one standard deviation is shown. FEA_1 is the result of a finite element model QLV model including tare strain with $\mu=0.11 \mathrm{MPa}$ and $E_{f}=128 \mathrm{MPa}$ (the collagen shear and fiber modulus respectively). FEA_2 does not include tare strain and $\mu=0.11 \mathrm{MPa}$ and $E_{f}=275 \mathrm{MPa}$. FEA $\_3$ does not include tare strain and $\mu=0.001$ $\mathrm{MPa}$ and $E_{f}=337 \mathrm{MPa}$. Table 4 lists the viscoelastic parameters calculated for each model. 


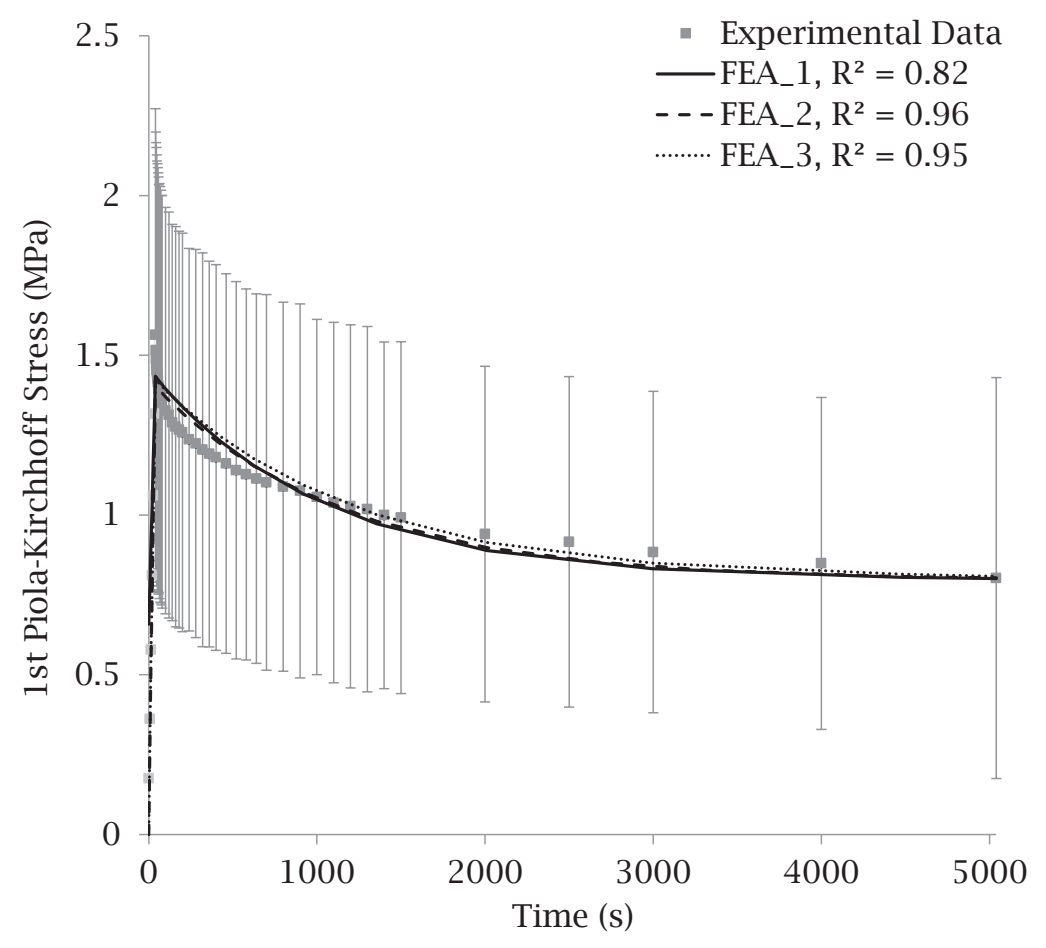

Figure 26: Comparison of QLV models curve fit to stress relaxation data from D0 middle layer bovine articular cartilage under tensile load. Test protocols are described in 32 .

Experimental data is the average of eight untreated specimens harvested from the patellarfemoral groove; one standard deviation is shown. FEA_1 is the result of a finite element model QLV model including tare strain with $\mu=0.11 \mathrm{MPa}$ and $E_{f}=525 \mathrm{MPa}$ (the collagen shear and fiber modulus respectively). FEA_2 does not include tare strain and $\mu=0.11 \mathrm{MPa}$ and $E_{f}=1131 \mathrm{MPa}$. FEA $\_3$ does not include tare strain and $\mu=$ $0.001 \mathrm{MPa}$ and $E_{f}=1227 \mathrm{MPa}$. Table 4 lists the viscoelastic parameters calculated for each model. 


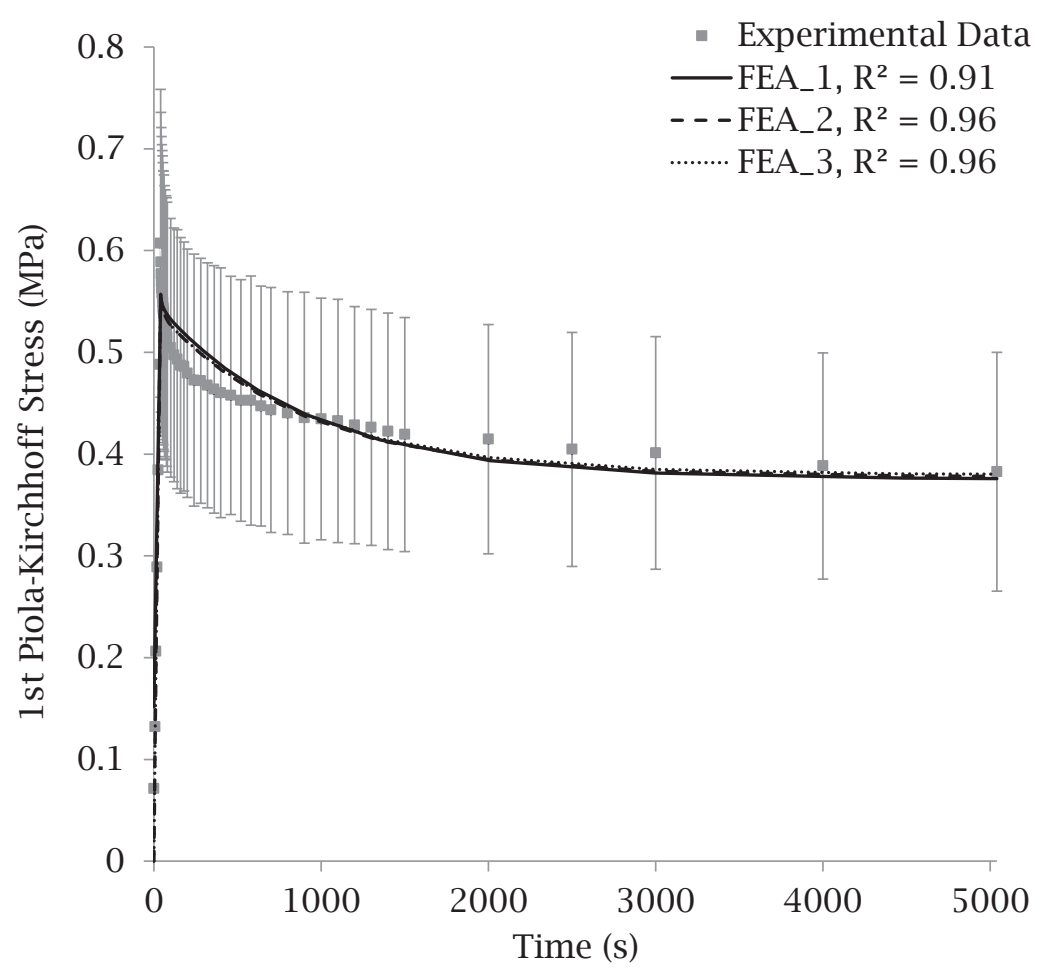

Figure 27: Comparison of QLV models curve fit to stress relaxation data from D12-TGF- $\beta 1$ superficial layer bovine articular cartilage under tensile load. Test protocols are described in 32. Experimental data is the average of six specimens harvested from the patellarfemoral groove; one standard deviation is shown. FEA_1 is the result of a finite element model QLV model including tare strain with $\mu=0.11 \mathrm{MPa}$ and $E_{f}=535 \mathrm{MPa}$ (the collagen shear and fiber modulus respectively). FEA_2 does not include tare strain and $\mu=0.11 \mathrm{MPa}$ and $E_{f}=767 \mathrm{MPa}$. FEA_3 does not include tare strain and $\mu=0.001$ $\mathrm{MPa}$ and $E_{f}=828 \mathrm{MPa}$. Table 4 lists the viscoelastic parameters calculated for each model. 


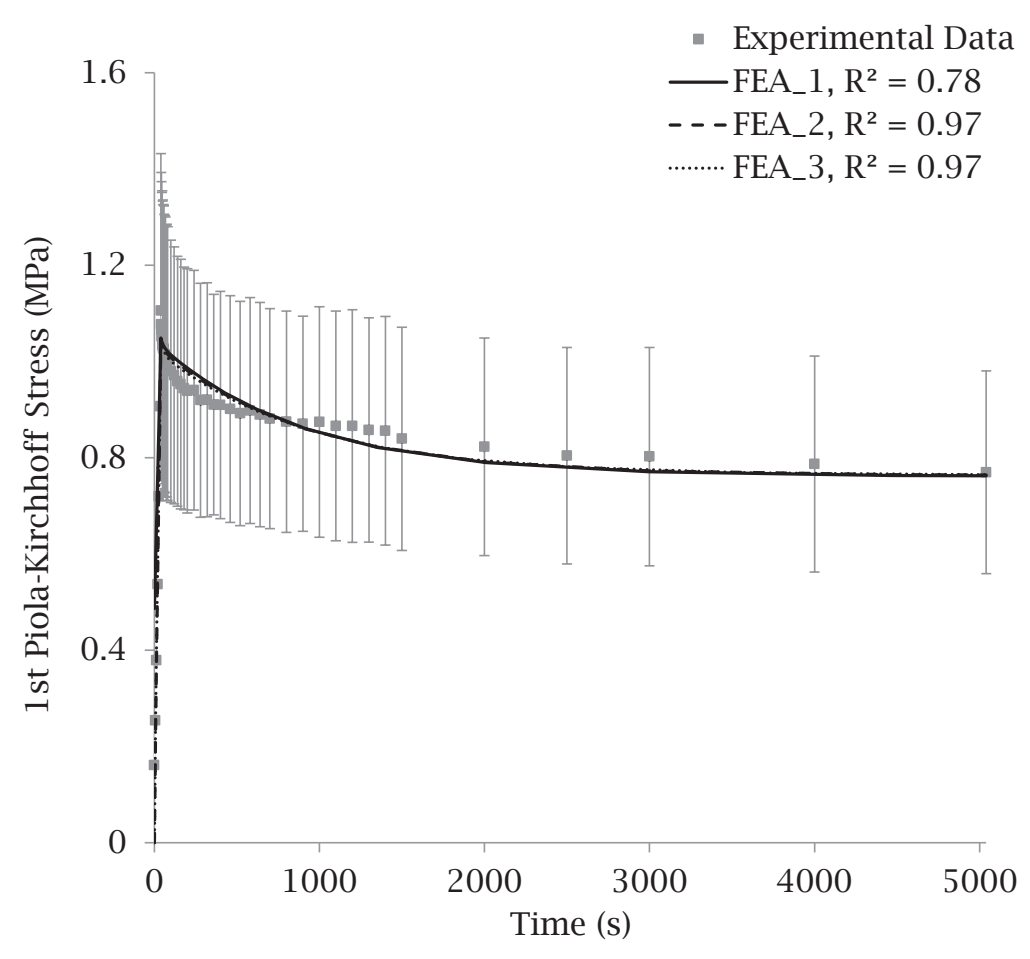

Figure 28: Comparison of QLV models curve fit to stress relaxation data from D12-TGF- $\beta 1$ middle layer bovine articular cartilage under tensile load. Test protocols are described in 32. Experimental data is the average of four specimens harvested from the patellarfemoral groove; one standard deviation is shown. FEA_1 is the result of a finite element model QLV model including tare strain with $\mu=0.11 \mathrm{MPa}$ and $E_{f}=1012 \mathrm{MPa}$ (the collagen shear and fiber modulus respectively). FEA_2 does not include tare strain and $\mu=0.11 \mathrm{MPa}$ and $E_{f}=2228 \mathrm{MPa}$. FEA $\_3$ does not include tare strain and $\mu=$ $0.001 \mathrm{MPa}$ and $E_{f}=2489 \mathrm{MPa}$. Table 4 lists the viscoelastic parameters calculated for each model. 
Table 11: Viscoelastic parameters for the QLV model for different tissues groups and tare considerations. These results are similar to Table 4 but also include results from tests including tare strain and with differing values of $\mu$. As explained in the Discussion section, tare strain is the recorded strain during experiment that was present before the specimen was stretched. It was thought that this strain was not actually being transferred to the specimen and was actually just from sag and jaw-slippage. From these results it was decided that the No-Tare, $\mu=0.001$ data produced the best fit to experimental data.

\begin{tabular}{|c|c|c|c|c|c|c|}
\hline Tissue & $\begin{array}{c}E_{f} \\
(\mathrm{MPa})\end{array}$ & $\begin{array}{c}\mu \\
(\mathrm{MPa})\end{array}$ & $\begin{array}{l}g_{1} \\
(-)\end{array}$ & $\begin{array}{l}\tau_{1} \\
(\mathrm{~s})\end{array}$ & $\begin{array}{l}g_{2} \\
(-)\end{array}$ & $\begin{array}{l}\tau_{2} \\
(\mathrm{~s})\end{array}$ \\
\hline $\begin{array}{l}\text { D0-S } \\
\text { (Tare) }\end{array}$ & 128 & 0.11 & 1.10 & 864 & 0.21 & 15 \\
\hline $\begin{array}{c}\text { D0-S } \\
\text { (No Tare) }\end{array}$ & 275 & 0.11 & 1.03 & 872 & 0.17 & 27 \\
\hline $\begin{array}{c}\text { D0-S } \\
\text { (No Tare) }\end{array}$ & 337 & 0.001 & 1.01 & 911 & 0.31 & 25 \\
\hline $\begin{array}{l}\text { D0-M } \\
\text { (Tare) }\end{array}$ & 525 & 0.11 & 1.05 & 961 & 0.03 & 17 \\
\hline $\begin{array}{c}\text { D0-M } \\
\text { (No Tare) }\end{array}$ & 1131 & 0.11 & 1.05 & 1024 & 0.10 & 17 \\
\hline $\begin{array}{c}\text { D0-M } \\
\text { (No Tare) }\end{array}$ & 1227 & 0.001 & 1.05 & 1128 & 0.18 & 27 \\
\hline $\begin{array}{c}\text { D12-TGF- } \beta 1-\mathrm{S} \\
\text { (Tare) }\end{array}$ & 535 & 0.11 & 0.55 & 860 & 0.12 & 16 \\
\hline $\begin{array}{c}\text { D12-TGF- } \beta 1-\mathrm{S} \\
\text { (No Tare) }\end{array}$ & 767 & 0.11 & 0.52 & 851 & 0.20 & 16 \\
\hline $\begin{array}{c}\text { D12-TGF- } \beta 1-\mathrm{S} \\
\text { (No Tare) }\end{array}$ & 828 & 0.001 & 0.51 & 850 & 0.19 & 16 \\
\hline $\begin{array}{c}\text { D12-TGF- } \beta 1-\mathrm{M} \\
(\text { Tare })\end{array}$ & 1012 & 0.11 & 0.46 & 844 & 0.12 & 16 \\
\hline $\begin{array}{c}\text { D12-TGF- } \beta 1-\mathrm{M} \\
\text { (No Tare) }\end{array}$ & 2228 & 0.11 & 0.46 & 844 & 0.12 & 16 \\
\hline $\begin{array}{c}\text { D12-TGF- } \beta 1-\mathrm{M} \\
\text { (No Tare) }\end{array}$ & 2489 & 0.001 & 0.45 & 871 & 0.15 & 17 \\
\hline
\end{tabular}




\section{E Standard Linear Solid - Pilot Study}

An initial study was done on the efficacy of using the standard linear solid to model collagen fibers. The derivation and results of that study are shown here.

\section{E.1 BACKGROUND}

The standard linear solid has been used to model micropipette aspiration of chondrocytes from human cartilage [47], creep indentation of bovine articular cartilage [83], indentation of demineralized human dentin [84], and stress relaxation of rabbit ligament [85]. Lei and Szeri [48] expanded this model to become nonlinear (having Young's modulus depend on strain) and tested the response in tension, unconfined compression, and indentation.

\section{E.2 Stress and Elasticity}

The model used to define the collagen fibers in this work implements the analogous form of linear springs and dashpots. For a linear spring (Figure 29) under uniform load, the constitutive equation can be defined as

$$
\sigma=E \epsilon
$$

where $\sigma$ is any measure of stress (Cauchy, first or second Piola-Kirchhoff, etc.), E is the elastic modulus of the spring, and $\epsilon$ is any measure of strain (Lagrangian, infinitesimal, etc.).

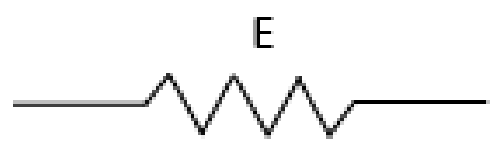

Figure 29: A linear spring with elastic modulus E

A linear dashpot (Figure 30) is a function of strain rate 


$$
\sigma=\eta \dot{\varepsilon}
$$

where $\eta$ is the damping coefficient.

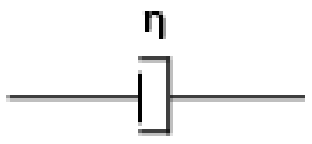

Figure 30: A linear damper with coefficient $\eta$

When two elements are in series, the stress across them is identical, but their strains (as well as strain rates) sum together. Similarly, when in parallel, the strain (or strain rate) across them is identical, whereas the stress is summed. Given the standard linear solid model of collagen as seen in Figure 31, the stress is derived as follows.

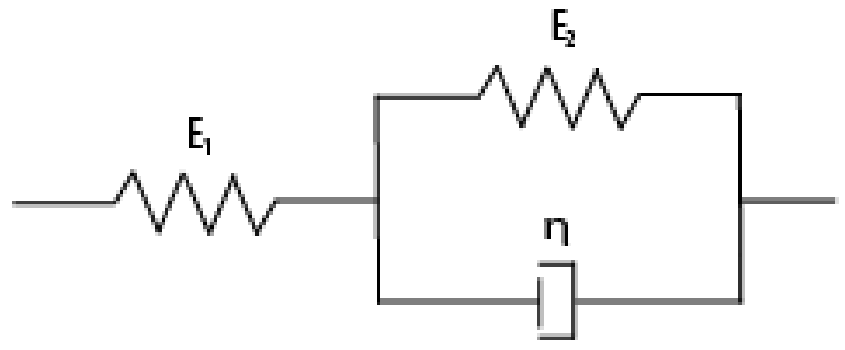

Figure 31: A standard linear solid (SLS) model.

First, recognize that

$$
\sigma=\sigma_{\text {spring }_{1}}=\sigma_{K V}
$$

and

$$
\varepsilon=\varepsilon_{\text {spring }_{1}}+\varepsilon_{K V}
$$


where $\sigma$ and $\varepsilon$ are arbitrary representations of stress and strain, respectively, and the subscript KV corresponds to the Kelvin-Voigt section of the SLS (the spring in parallel with the dashpot. From the standard definition of a linear spring,

$$
\varepsilon_{\text {spring }}=\frac{\sigma}{E_{1}}
$$

Further, $\sigma_{K V}$ is the sum of the stress of each component

$$
\sigma_{K V}=E_{2} \varepsilon_{K V}+\eta \dot{\varepsilon}_{K V}
$$

where $\dot{\varepsilon}_{K V}$ is the strain rate of the KV section. Equation 97 can be rewritten as

$$
\varepsilon_{K V}=\frac{\sigma}{\left\{E_{2}+\eta \delta_{t}\right\}}
$$

where $\delta_{t}$ is the time derivative operator, defined as

$$
\delta_{t}=\frac{\partial}{\partial t}
$$

Using 98 and 96,95 can be rewritten as

$$
\varepsilon=\frac{\sigma}{E_{1}}+\frac{\sigma}{\left\{E_{2}+\eta \delta_{t}\right\}}
$$

Multiplying through by $E_{1}$ and the bracketed operator and distributing the bracketed operator, 100 becomes

$$
E_{1} E_{2} \varepsilon+E_{1} \eta \dot{\varepsilon}=\left(E_{1}+E_{2}\right) \sigma+\eta \dot{\sigma} .
$$

Solving for $\sigma$ yields

$$
\sigma=\frac{1}{E_{1}+E_{2}}\left(E_{1} \eta \dot{\varepsilon}+E_{1} E_{2} \varepsilon-\eta \dot{\sigma}\right) .
$$

where $\eta, E_{1}$, and $E_{2}$ reference the mechanical constants as seen in Figure 31.

Next, consider the central differencing operators 


$$
\begin{gathered}
\dot{f}\left(t_{1}+\frac{1}{2} \Delta t\right)=\frac{\Delta f}{\Delta t} \\
f\left(t_{1}+\frac{1}{2} \Delta t\right)=f\left(t_{1}\right)+\frac{\Delta f}{2}
\end{gathered}
$$

where $f$ is some arbitrary function of $t, t_{1}$ is the time at the beginning of the increment, $t_{2}$ is the time at the end of the increment, $\Delta t$ is the time increment, such that

$$
\Delta t=t_{2}-t_{1},
$$

and $\Delta f$ is the change in $f$ during $\Delta t$. Let us redefine Equation 102 as

$$
\sigma\left(t_{1}+\frac{1}{2} \Delta t\right)=\frac{1}{E_{1}+E_{2}}\left[E_{1} \eta \dot{\varepsilon}\left(t_{1}+\frac{1}{2} \Delta t\right)+E_{1} E_{2} \varepsilon\left(t_{1}+\frac{1}{2} \Delta t\right)-\eta \dot{\sigma}\left(t_{1}+\frac{1}{2} \Delta t\right)\right] .
$$

Then using Equation 103 and 104,

$$
\begin{gathered}
\sigma\left(t_{1}+\frac{1}{2} \Delta t\right)=\sigma\left(t_{1}\right)+\frac{\Delta \sigma}{2}, \\
\dot{\varepsilon}\left(t_{1}+\frac{1}{2} \Delta t\right)=\frac{\Delta \varepsilon}{\Delta t}, \\
\varepsilon\left(t_{1}+\frac{1}{2} \Delta t\right)=\varepsilon\left(t_{1}\right)+\frac{\Delta \varepsilon}{2},
\end{gathered}
$$

and

$$
\dot{\sigma}\left(t_{1}+\frac{1}{2} \Delta t\right)=\frac{\Delta \sigma}{\Delta t}
$$

so that Equation 106 becomes

$$
\sigma\left(t_{1}\right)+\frac{\Delta \sigma}{2}=\frac{1}{E_{1}+E_{2}}\left[E_{1} \eta \frac{\Delta \varepsilon}{\Delta t}+E_{1} E_{2}\left(\varepsilon\left(t_{1}\right)+\frac{\Delta \varepsilon}{2}\right)-\eta \frac{\Delta \sigma}{\Delta t}\right] .
$$

Solving for $\Delta \sigma$, 


$$
\Delta \sigma=\frac{1}{\frac{E_{1}+E_{2}}{2}+\frac{\eta}{\Delta t}}\left[E_{1} \eta \frac{\Delta \varepsilon}{\Delta t}+E_{1} E_{2}\left(\varepsilon\left(t_{1}\right)+\frac{\Delta \varepsilon}{2}\right)-\left(E_{1}+E_{2}\right) \sigma\left(t_{1}\right)\right] .
$$

The stress at the current time is then solved for as

$$
\sigma\left(t_{2}\right)=\sigma\left(t_{1}\right)+\Delta \sigma
$$

Using Equation 112, Equation 113 becomes

$$
\sigma_{2}=\left(1-\frac{E_{1}+E_{2}}{\frac{E_{1}+E_{2}}{2}+\frac{\eta}{\Delta t}}\right) \sigma_{1}+\frac{1}{\frac{E_{1}+E_{2}}{2}+\frac{\eta}{\Delta t}}\left[E_{1} E_{2} \varepsilon_{1}+\left(\frac{E_{1} \eta}{\Delta t}+\frac{E_{1} E_{2}}{2}\right) \Delta \varepsilon\right]
$$

where $\sigma_{2}$ and $\sigma_{1}$ correspond to the stress at the end and beginning of the time increment respectively, $\varepsilon_{1}$ corresponds to the strain at the beginning of the time increment, $\Delta \varepsilon$ is the change in strain over the time increment, and $\Delta t$ is the time increment.

Stress in the collagen network is calculated by using second Piola-Kirchhoff stress and Lagrangian strain in place of $\sigma$ and $\varepsilon$ in Equation 114 and inserting this result in Equation 20 in the place of $S_{n}$. Second Piola-Kirchhoff stress and Lagrangian strain are used in place of Cauchy stress and logarithmic strain because $\frac{\mathrm{d} S}{\mathrm{~d} t}$ and $\boldsymbol{E}$ are objective under any deformation (the qualitative and quantitative definition of these properties remains unchanged for any deformation). If we implement these changes and use Equation 20, we can calculate the collagen stress at the current time as

$$
\boldsymbol{S}^{C O L}=\int_{V} R H S_{2}(\boldsymbol{n} \otimes \boldsymbol{n}) \mathrm{d} V
$$

From Equation $21 \frac{\partial S_{n}}{\partial \boldsymbol{E}}$ can be separated into

$$
\frac{\partial S_{n}}{\partial \boldsymbol{E}}=\frac{\partial S_{n}}{\partial E_{n}} \frac{\partial E_{n}}{\partial \boldsymbol{E}}
$$


where $E_{n}$ is the Lagrangian fiber strain. $\frac{\partial S_{n}}{\partial E_{n}}$ is approximated as

$$
\frac{\partial S_{n}}{\partial E_{n}} \approx \frac{\Delta \sigma}{\Delta \varepsilon}
$$

where $\frac{\Delta \sigma}{\Delta \varepsilon}$ is calculated from Equation 114 as

$$
\frac{\Delta \sigma}{\Delta \varepsilon}=\left[\frac{1}{\frac{E_{1}+E_{2}}{2}+\frac{\eta}{\Delta t}}\left(\frac{E_{1} \eta}{\Delta t}+\frac{E_{1} E_{2}}{2}\right)\right],
$$

and $E_{n}=\boldsymbol{E} \boldsymbol{n} \cdot \boldsymbol{n}$ so that

$$
\frac{\partial E_{n}}{\partial \boldsymbol{E}}=\boldsymbol{n} \otimes \boldsymbol{n}
$$

Combining these results, $\tilde{\mathbb{C}}^{C O L}$ becomes

$$
\tilde{\mathbb{C}}^{C O L}=\int_{V} R H \frac{\Delta \sigma}{\Delta \varepsilon}(\boldsymbol{n} \otimes \boldsymbol{n}) \otimes(\boldsymbol{n} \otimes \boldsymbol{n}) \mathrm{d} V .
$$

Remember that $\sigma$ and $\varepsilon$ in the preceding equations are arbitrary definitions of stress. Therefore, a substitution of second Piola-Kirchhoff stress and Lagrangian strain is acceptable.

\section{E.3 Exact Solution to Differential Equation}

The exact solution to the differential equation describing the stress-strain relationship is shown here. Given the differential equation

$$
E_{1} E_{2} \varepsilon+E_{1} \eta \dot{\varepsilon}=\left(E_{1}+E_{2}\right) \sigma+\eta \dot{\sigma}
$$

first recognize that strain is defined piecewise as

$$
\varepsilon(t)=\left\{\begin{array}{cc}
A t & 0 \leq t \leq t_{f} \\
\varepsilon_{f} & t>t_{f}
\end{array}\right.
$$


where $A$ is the strain rate, $t_{f}$ is the ramp time, and $\varepsilon_{f}$ is the maximum strain. The differential equation must be solved piecewise as well. For the first time interval $\left(0 \leq t \leq t_{f}\right)$, note the following property of ordinary differential equations.

Consider some ODE, represented as

$$
C_{1} f+C_{2} \dot{f}=C_{3} g+C_{4} \dot{g}
$$

where $f$ and $g$ are arbitrary functions of time, and $C_{\alpha}$ are coefficients. The solution can be obtained by first finding the solution to

$$
C_{1} f+C_{2} \dot{f}=g
$$

which we'll call $f^{\prime}$ and plugging the result into

$$
f=C_{3} f^{\prime}+C_{4} \dot{f}^{\prime}
$$

Using this property, we will first solve

$$
\dot{\sigma}^{\prime}+\frac{E_{1}+E_{1}}{\eta} \sigma^{\prime}=A t
$$

The first step is to find the homogeneous solution, by setting the equation equal to zero. Then

$$
\lambda+\frac{E_{1}+E_{2}}{\eta}=0
$$

Solving for $\lambda$

$$
\lambda=-\frac{E_{1}+E_{2}}{\eta}
$$

so that the homogeneous solution is

$$
\sigma_{H}^{\prime}=C_{1} e^{-\frac{E_{1}+E_{2}}{\eta} t} .
$$


Assume that the particular solution is of the form

$$
\sigma_{P}^{\prime}=K_{1} t+K_{2}
$$

Then

$$
\left(E_{1}+E_{2}\right)\left(K_{1} t+K_{2}\right)+\eta K_{1}=A t .
$$

Equating like terms

$$
\begin{gathered}
\left(E_{1}+E_{2}\right) K_{1}=A \\
\left(E_{1}+E_{2}\right) K_{2}+\eta K_{1}=0 .
\end{gathered}
$$

Solving simultaneously for $K_{1}$ and $K_{2}$,

$$
\begin{gathered}
K_{1}=\frac{A}{E_{1}+E_{2}} \\
K_{2}=-\frac{A \eta}{\left(E_{1}+E_{2}\right)^{2}} .
\end{gathered}
$$

The particular solution is then

$$
\sigma_{P}^{\prime}=\frac{A}{E_{1}+E_{2}} t-\frac{A \eta}{\left(E_{1}+E_{2}\right)^{2}}
$$

$\sigma^{\prime}$ is then solved for as the addition of $\sigma_{H}^{\prime}$ and $\sigma_{P}^{\prime}$

$$
\sigma^{\prime}=C_{1} e^{-\frac{E_{1}+E_{2}}{\eta} t}+\frac{A}{E_{1}+E_{2}} t-\frac{A \eta}{\left(E_{1}+E_{2}\right)^{2}} .
$$

The solution to the original differential equation is then found by

$$
\sigma=E_{1} \eta \dot{\sigma}^{\prime}+E_{1} E_{2} \sigma^{\prime}
$$

or 


$$
\begin{aligned}
\sigma_{1}= & E_{1} \eta\left[-\frac{E_{1}+E_{2}}{\eta} C_{1} e^{-\frac{E_{1}+E_{2}}{\eta} t}+\frac{A}{E_{1}+E_{2}}\right] \\
& +E_{1} E_{2}\left[C_{1} e^{-\frac{E_{1}+E_{2}}{\eta} t}+\frac{A}{E_{1}+E_{2}} t-\frac{A \eta}{\left(E_{1}+E_{2}\right)^{2}}\right] .
\end{aligned}
$$

Using the initial condition, $\sigma(0)=0, C_{1}$ can be solved for as

$$
C_{1}=\frac{A\left[\frac{E_{1} E_{2} \eta}{\left(E_{1}+E_{2}\right)^{2}}-\frac{E_{1} \eta}{E_{1}+E_{2}}\right]}{-E_{1}\left(E_{1}+E_{2}\right)+E_{1} E_{2}} .
$$

Similarly for $t>t_{f}$

$$
\sigma_{2}=C_{2} e^{-\frac{E_{1}+E_{2}}{\eta} t}+\frac{\varepsilon_{f} E_{1} E_{2}}{E_{1}+E_{2}}
$$

where $C_{2}$ is

$$
C_{2}=\frac{\sigma_{1}\left(t_{f}\right)-\frac{\varepsilon_{f} E_{1} E_{2}}{E_{1}+E_{2}}}{e^{-\frac{E_{1}+E_{2}}{\eta} t_{f}}} .
$$

Here, $\sigma_{1}\left(t_{f}\right)$ is the stress evaluated from the previous solution at the time to maximum strain.

\section{E.4 MeTHODS}

For the standard linear solid, it is necessary to save the fiber stress from the previous time increment for each fiber, therefore state variables for each fiber direction were implemented. In order to complete the integration in Equation 115, the following loop was coded in the UMAT.

The fiber stress is found using Equation 114, which is input into

$$
\boldsymbol{S}^{C O L}=\sum_{i=1}^{p} R H \sigma_{2}(\boldsymbol{N} \otimes \boldsymbol{N}) \mathrm{d} V
$$

where $\mathrm{p}$ is the number of pyramids (from the unit sphere), which forms Equation 115. After the collagen stress loop, $\boldsymbol{S}^{C O L}$ is transformed into Cauchy stress according to Equation 10. Equation 120 is solved similarly as 


$$
\tilde{\mathbb{C}}^{C O L}=\sum_{i=1}^{p} R H \frac{\Delta \sigma}{\Delta \varepsilon}(\boldsymbol{N} \otimes \boldsymbol{N}) \otimes(\boldsymbol{N} \otimes \boldsymbol{N}) \mathrm{d} V
$$

which is input into Equation 12 to find the Jaumann-Kirchhoff elasticity tensor.

All other methods are identical to the methods set forth in Section 4.

\section{E.5 Results}

Results for the SLS model are shown in Figure 32.

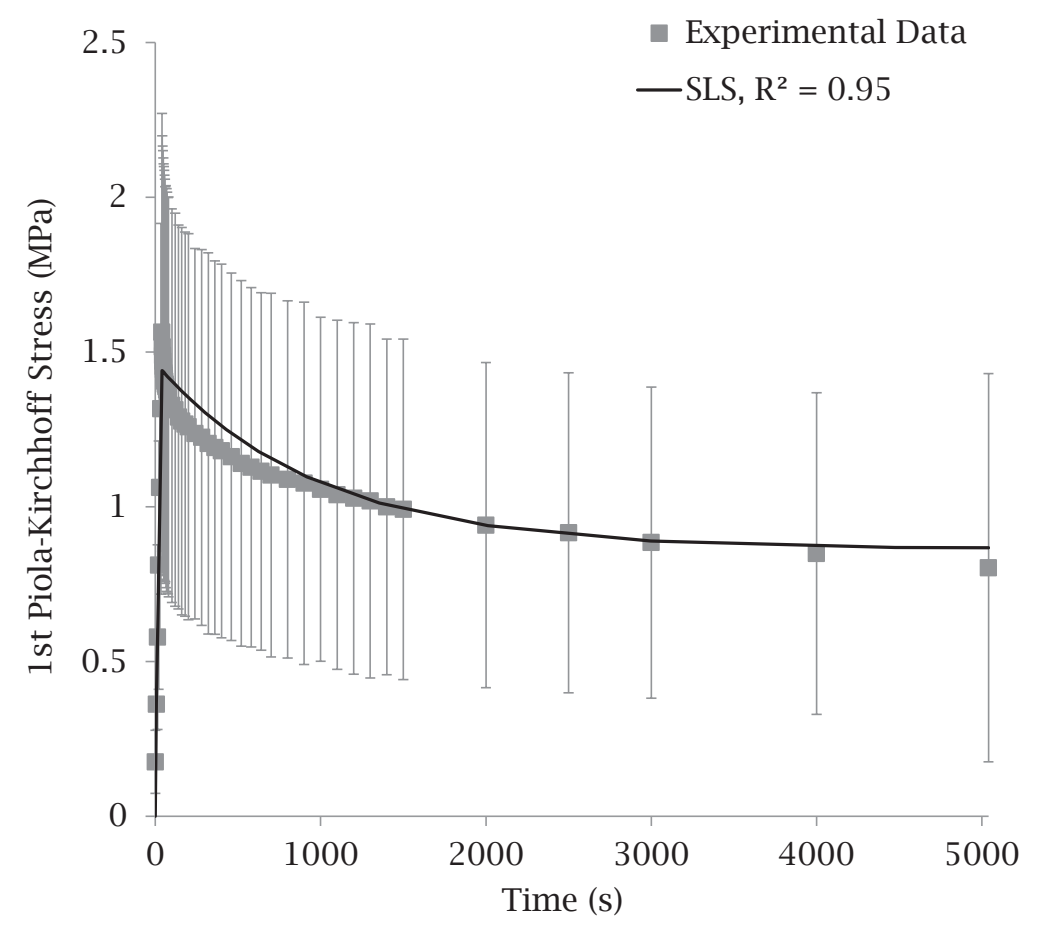

Figure 32: Standard linear solid model curve fit to average D0-M stress relaxation data. Experimental data was the average of 8 untreated explants harvested from the middle layer of the patellarfemoral groove of bovine articular cartilage. The tensile test consisted of a 40 s ramp loading to $5 \%$ strain followed by a 5000 s relaxation period. Results of the SLS model produced $E_{1}=6000 \mathrm{MPa}, E_{2}=5666 \mathrm{MPa}$, and $\eta=1.03 \mathrm{E} 7 \mathrm{~s}$. The coefficient of determination for this model is $R^{2}=0.95$.

\section{E.6 Discussion}

A standard linear solid was studied as a viscoelastic model for collagen fibers in the mixture model of articular cartilage. Although the results of Figure 32 show relatively good fit, it was noted that the experimental data seemed to exhibit a 
relaxation profile more suited to a two relaxation term model. The standard linear solid is a simpler representation of viscoelasticity and may still be considered an effective model if future studies require such simplicity. 